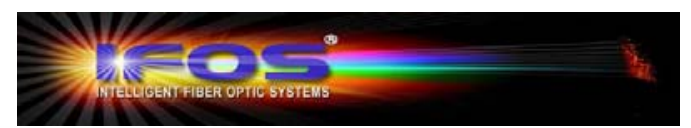

2363 Calle Del Mundo

Santa Clara, CA 95054

www.ifos.com
PI: DR. VAHID SOTOUDEH

E-mail: vs@ifos.com

Phone: (408) 565-9000

Fax: (408) 565-9003

\title{
Fiber-Optic Defect and Damage Locator System for Wind Turbine Blades
}

\author{
DOE STTR GRANT NO.: DE-SCOOO1020 \\ (Performance Period: July 20th, 2009 - April 19th, 2010) \\ Phase-I Final Report
}

October 30th, 2010

DOE Program Manager

Mark Higgins

Tel: 202-287-5213

Mark.Higgins@ee.doe.gov

DOE Technical Monitor John Meissner

New West Technologies

U.S. Department of Energy (EERE)

Wind and Hydropower Technologies Program

Office: (202) 287-5777

Cell: (858) 344-4366

Fax: (202) 586-5124

John.Meissner@ee.doe.gov

The data contained in pages 1-81 of this application have been submitted in confidence and contain trade secrets or proprietary information, and such data shall be used or disclosed only for evaluation purposes, provided that if this applicant receives an award as a result of or in connection with the submission of this application, DOE shall have the right to use or disclose the data herein to the extent provided in the award. This restriction does not limit the Government's right to use or disclose data obtained without restriction from any source, including the applicant. 


\section{Fiber-Optic Defect and Damage Locator System for Wind Turbine Blades}

Intelligent Fiber Optic Systems Corporation (IFOS)

Principal Investigator (PI): Dr. Vahid Sotoudeh

\section{PROJ ECT SUMMARY}

\section{Statement of the problem or situation that is being addressed}

The capability of locating and quantifying structural damage is one of the most important requirements for verifying and maintaining safety and integrity of wind turbine blades. A robust and reliable nondestructive defect and damage identification and assessment capability is essential to predictable maintenance and disaster avoidance.

\section{General statement of how this problem is being addressed}

IFOS in collaboration with Auburn University demonstrated the feasibility of a Fiber Bragg Grating (FBG) integrated sensor system capable of providing real time in-situ defect detection, localization and quantification of damage. In addition, the system is capable of validating wind turbine blade structural models, using recent advances in non-contact, non-destructive dynamic testing of composite structures. This new generation method makes it possible to analyze wind turbine blades not only non-destructively, but also without physically contacting or implanting intrusive electrical elements and transducers into the structure.

\section{Phase I Results}

Phase I successfully demonstrated the feasibility of the technology with the construction of a 1.5 $\mathrm{kHz}$ sensor interrogator and preliminary instrumentation and testing of both composite material coupons and a wind turbine blade.

\section{Commercial Applications and Other Benefits}

Wind turbines are increasingly populating the landscape offering an additional source of electric power. The wind turbine sensor market continues to rapidly expand with tremendous potential. These project findings can enable performance optimization of both land-based and off-shore wind turbines through early defect and damage detection in wind turbine blades, potentially reduce the end cost per watt and enable this current solution to gain greater momentum. In addition, the IFOS solution can lead improved reliability and increased efficiency of operational wind plants as well as provide an economically viable technology. IFOS has obtained letters of support and commitment from strategic partners and collaborators.

\section{Key Words}

Fiber optic sensors, Wind turbines, Wind turbine blades, Wind turbine blade damage, Wind turbine blade reliability, Energy security, Environment

\section{Summary for Members of Congress (Max. 50 words)}

Increased harnessing of wind power benefits the planet by providing renewable energy reducing reliance on fossil fuels. The proposed novel sensing system contributes to our Nation's security and environment by detecting defects in wind turbine blades, optimizing their performance, and preventing costly turbine shut downs due to unpredictable blade failures. 
1 SIGNIFICANCE, BACKGROUND INFORMATION, AND TECHNICAL APPROACH .........................4

1.1 IDENTIFICATION AND SIGNIFICANCE OF PROBLEM OR OPPORTUNITY .........................................................

1.1.1 Wind Turbine Blade Ice Problems, Pitch Control \& Retrofitting .............................................................5

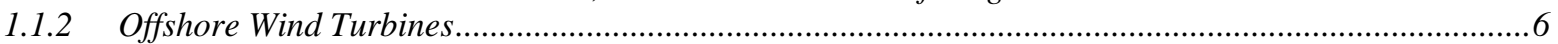

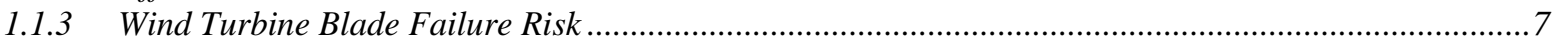

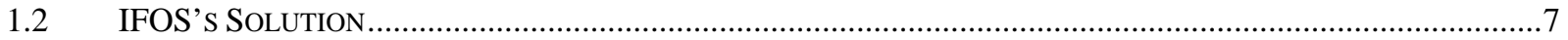

1.2.1 Fiber Bragg Grating (FBG) Sensors .......................................................................................

1.2.2 Composite Materials with Embedded FBG Sensors .......................................................................10

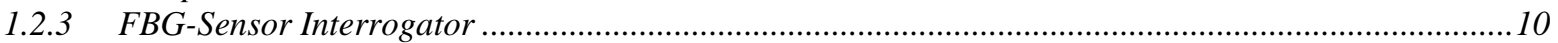

1.2.4 Competitive Advantages of the IFOS Approach .........................................................................11

1.2.5 Related Research Work Conducted by the Project Team .....................................................................11

1.2.6 Research Work Available in Archived Literature .................................................................................12

2 ANTICIPATED PUBLIC BENEFITS.............................................................................................................14

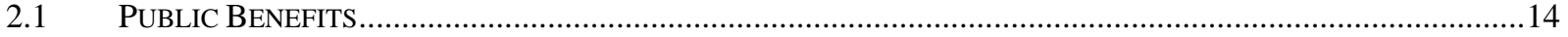

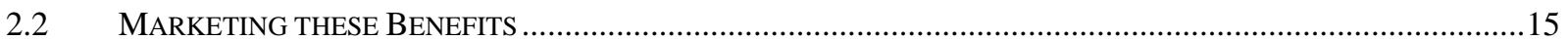

3 DEGREE TO WHICH PHASE I HAS DEMONSTRATED TECHNICAL FEASIBILITY .....................15

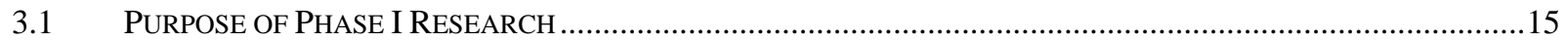

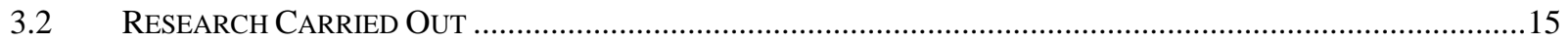

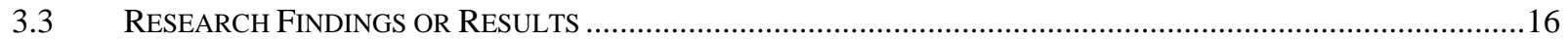

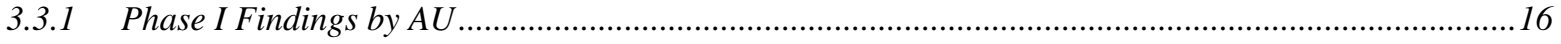

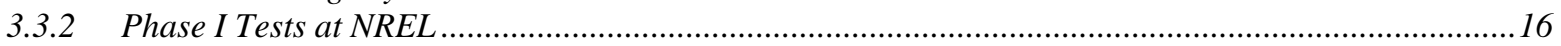

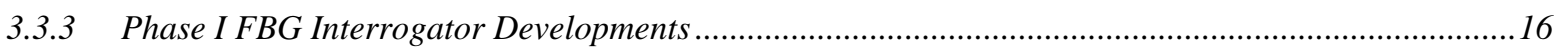

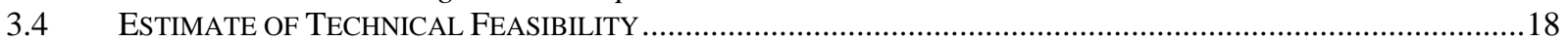

3.5 DEgREe TO WHICH THE PHASE I OBJECTIVES HAVE BEEN MET........................................................18

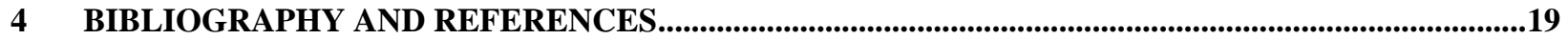

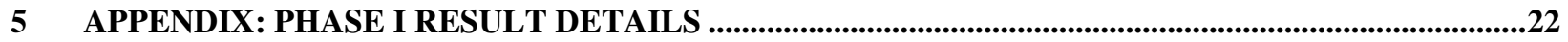

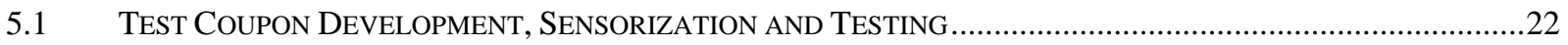

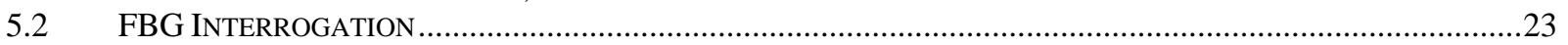

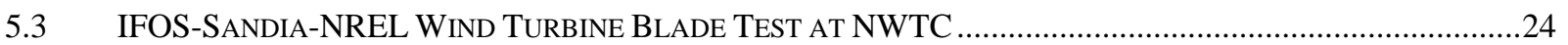

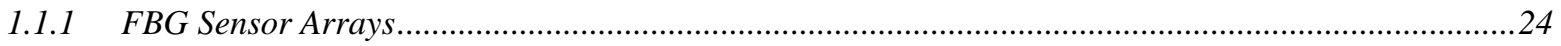

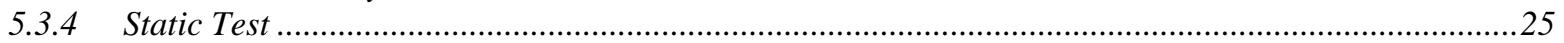

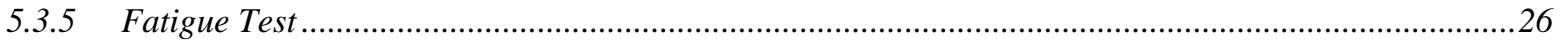

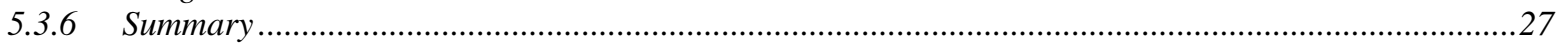

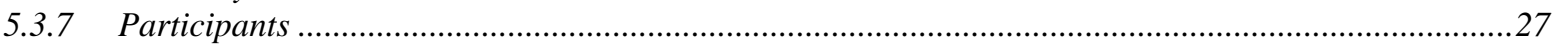

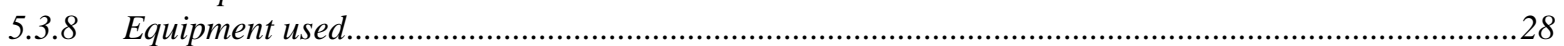

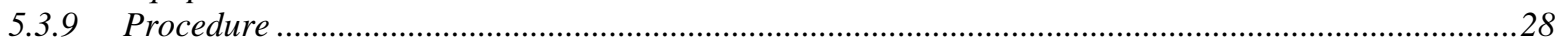

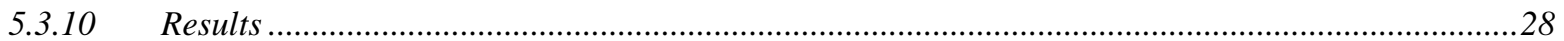

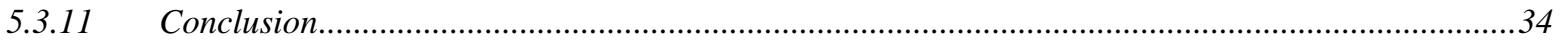

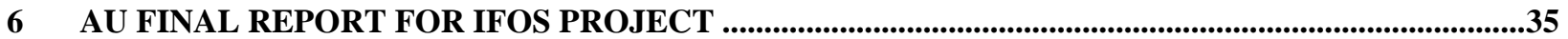




\section{Intelligent Fiber Optic Systems Corp. (IFOS) DOE STTR GRANT No.: DE- SC0001020 \\ Interim Report \#4}

\section{Significance, Background Information, and Technical Approach}

This Small Business Technology Transfer Research (STTR) Phase I project granted by the Department of Energy (DOE) SBIR/STTR program supported Intelligent Fiber Optic Systems Corporation (IFOS) ${ }^{1}$ to develop a Phase I model of a multiplexed fiber-optic-based sensor system to meet DOE's requirements for wind turbine blades in-situ testing for reliability assurance. This project was based on the original Phase I Topic 20, "WIND ENERGY TECHNOLOGY DEVELOPMENT," subtopic b) Components Reliability. The IFOS innovation demonstrated the possibility of analyzing complex wind turbine blade structures not only nondestructively, but also without implanting electrical elements into the blade structure under test. IFOS demonstrated that its solution can withstand the harsh and demanding environment in which different wind turbines are installed. IFOS performed tests both on composite material coupons and a full-sized 9-meter blade in Phase I, which will ease the transition to prototype development in the future. IFOS, which has a proven track record in designing and developing sensor systems for harsh environments, collaborated with several organizations that are highly experienced in wind turbine in wind turbine research and development, each offered unique expertise in areas that had direct impact on the goals set for the project. Partners and participants in this project include:

- Auburn University (AU) - a team led by Professors Gowayed and Flowers of the Departments of Polymer and Fiber Engineering and Mechanical Engineering - STTR Research Institute partner on this project - with well-known expertise in the fields of polymer and fiber science, materials, mechanical and civil engineering, who bring their decades long collective track record in finite element analysis to support the extensive modeling required for an effective structural health monitoring project

- the National Wind Technology Center (NWTC) of DOE National Renewable Energy Laboratory (NREL) - a team led by Mr. Scott Hughes who manages technical activities in the structural testing laboratories at the NWTC, leads blade methodology development efforts at the laboratory, developing advanced mechanical systems and approaches for testing of large wind blades, has been the technical lead in over 70 full scale structural tests of wind turbine blades

- Sandia National Labs (SNL) - Wind Power Technologies Department - a team led by Mr. Mark Rumsey who is responsible for the research and development of sensors for the next generation of wind turbine structures with an emphasis on wind turbine blades, the research and development of nondestructive testing and health monitoring techniques as they apply to wind energy applications, and the facilitation of field-testing activities

\footnotetext{
${ }^{1}$ The data contained in pages 1-81 of this application have been submitted in confidence and contain trade secrets or proprietary information, and such data shall be used or disclosed only for evaluation purposes, provided that if this applicant receives an award as a result of or in connection with the submission of this application, DOE shall have the right to use or disclose the data herein to the extent provided in the award. This restriction does not limit the Government's right to use or disclose data obtained without restriction from any source, including the applicant.
} 


\section{Intelligent Fiber Optic Systems Corp. (IFOS) DOE STTR GRANT NO.: DE- SC0001020 \\ Interim Report \#4}

\subsection{Identification and Significance of Problem or Opportunity}

As wind energy systems increase their penetration into the national electrical power base, longterm reliability of wind turbines becomes of ever greater importance. New tools and methods are needed to perform real time and predictive condition monitoring on major wind turbine subsystems, including blades, gearboxes, towers, and generators. Moisture absorption, fatigue, wind gusts, and lightning strikes can be factors that damage wind turbine blades. In wind farms, aerodynamic interaction between different turbines can also cause unpredictable and excessive loads on the blades. Such stressors, couple with normal fatigue cycles and aerodynamic loads caused by changing gravity moments to cause fatigue damage. Moreover, these blades are massive structures with a 1.5-2.5 MW turbine weighing some 22,000 lbs and blades 10" thick at base, usually made of fiberglass, vinyl esters and epoxies with low specific modulus and low natural frequencies. Under loading, the deflections and strains of the cantilevered blade can be quite large. While the design lifetime for blades is from 10 to 30 years, predicting the exact fatigue life is difficult. If fatigue-damaged rotor blades fail, they can cause catastrophic damage to a wind turbine. The cost of such damage is not limited to equipment replacement but includes potential loss of revenue due to down-time, cost of rolling a crane (perhaps $\$ 100 \mathrm{k}$ ) to difficult terrain and repair costs that require repeated fielding of maintenance crews etc. Repair of a wind turbine is further complicated if it is located off shore. To ensure peak performance and maintain high turbine availability, a method is needed for continuous monitoring of the condition of the blade, along with warning of possible failure in real-time to permit on the fly adjustments such as, e.g., blade attack angle adjustments or gear change.

One of the approaches to reducing the cost for corrective maintenance for the wind turbines involves condition monitoring for early failure detection. If failures can be detected at an early stage, the severity of consequent damage can be substantially reduced so repairs can be performed less expensively. Offshore wind turbines will, however, benefit the most from early failure detection capability, since repairs can be better planned - a crucial aspect for more challenging deployment environments. This will lead to considerably shorter downtimes and lower revenue losses [Verbruggen, 2003].

Online monitoring of wind turbine blades records their health state by detecting minor changes in the structural and material strain response. Strain data offer vital information for safe operation allowing for informed maintenance decisions as well as avoidance of possible catastrophic failures. Accurate detection of strain values, as an indicator of the health state of the structure, is crucial to the success of the monitoring process. To this end, the proposed blade monitoring system comprises two key components:

- A highly robust Fiber Bragg Grating (FBG) integrated sensor system capable of providing real time in-situ strain measurements.

- An understanding of the significance and implications of these strain measurements on the overall behavior of the blade structure and material under various operating conditions.

\subsubsection{Wind Turbine Blade Ice Problems, Pitch Control \& Retrofitting}

Ice Problem: Currently, wind turbines are shut down as a result of ice formation on the blades (for public safety reasons, for example). Current detection methods do not properly indicate 


\section{Intelligent Fiber Optic Systems Corp. (IFOS) DOE STTR GRANT No.: DE- SC0001020 \\ Interim Report \#4}

when the sun has melted ice on the blades. Thus, a significant monetary penalty is associated with failing to turn on the turbine promptly once the ice melts. IFOS' sensors on both sides of the blades allow for immediate detection of melting of the ice (through monitoring the change in the ambient vibration frequency of the blade), thus enabling turbines to operate for many hours during which the turbines would otherwise be shut down.

Pitch Control: Non-uniform wind loading across a wind turbine blade as well as amongst the blades produces uneven loads on the blades as well as on the drive shaft and turbine structure. This condition leads to excessive wear-and-tear in the wind turbine, reducing its useful life and increasing the number of unplanned shut downs for maintenance. The proposed IFOS sensor technology provides a blade load monitoring capability as needed for active blade pitch control, thereby reducing the loading fluctuation effects on the wind turbine components.

Retrofitting: As the number of installed wind turbines increases, the number of wind turbine blades requiring repair and retrofit also increases. Adding a condition monitoring system (CMS) when the blade is in for repair and retrofit presents an opportunity that IFOS intends to pursue in collaboration with KCWG, who are known for their comprehensive repair and retrofit capabilities. An important benefit of this development effort is that it can be applied to both new and current wind turbine installations.

Although land-based wind turbines are becoming increasingly well established, offshore wind turbines present significant additional technical and economic challenges, as described next.

\subsubsection{Offshore Wind Turbines}

IFOS considers it likely that there will be a much greater need for CMS offshore given that there are more wind turbine assets at risk, capital investment is much higher and maintenance costs are generally greater. Aspects of the considerations include:

- On the US East Coast, offshore wind resources are very significant and there is increasing inclination to proceed with installation of offshore wind farms.

- However, the requirements are much more stringent especially for potential US deep water platforms. In particular,

o Durability and maintenance are critical issues.

o The industry has not yet matured for offshore.

o While there is much more at risk offshore, and installation costs and maintenance costs are higher, the biggest hurdle for offshore adoption in the US has been policy. Involvement of local groups such as the Maine Maritime Academy (MMA) in developing new policies for offshore wind development will be beneficial.

o The marine environment, mineral resources, and shipping are all important considerations that offshore wind needs to take into account.

- The 2.5MW wind turbines look well suited for offshore installation.

- A complex permit process has stalled large scale development in US.

- In Europe, offshore installation in the North Sea and other locations has been facilitated by relatively shallow seabeds. By contrast, around most US shores, the seabed drops off much more quickly as one moves away from shoreline, and deep water installation is required in most locations.

- For deep-water installations involving mono pole or some other foundation that penetrates into the seabed, the system is more complicated and more expensive

- Floating platforms for deep water applications present their own installation and operation challenges:

0 Wave action and added loads from water, in addition to the wind

o Higher levels of sustained wind loading than for land-based turbines

IFOS Proprietary Fiber-Optic Defect $\&$ Damage Locator System for Wind 


\section{Intelligent Fiber Optic Sy stems Corp. (IFOS) DOE STTR GRANT No.: DE-}

SC0001020

Interim Report \#4

o New structural design requirements for deep-water offshore wind turbines in comparison with land and fixed foundation offshore machines.

- Environmental conditions will guide selection of proper architecture

\subsubsection{Wind Turbine Blade Failure Risk}

The principal investigator of this proposal attended the first wind turbine CMS workshop by NREL (Bloomfield, CO, October 8-9, 2009).

Figure 1 shows a presentation slide [Walford, 2009] from this workshop showing relative risks of different wind turbine components failures and their associated costs. It can be seen that the blades failures are more likely to occur after the wind turbine warranty period expiration date with the relative cost of even minor repairs to be the highest.

\section{Figure 1 Wind Turbine Components Failure Risk}

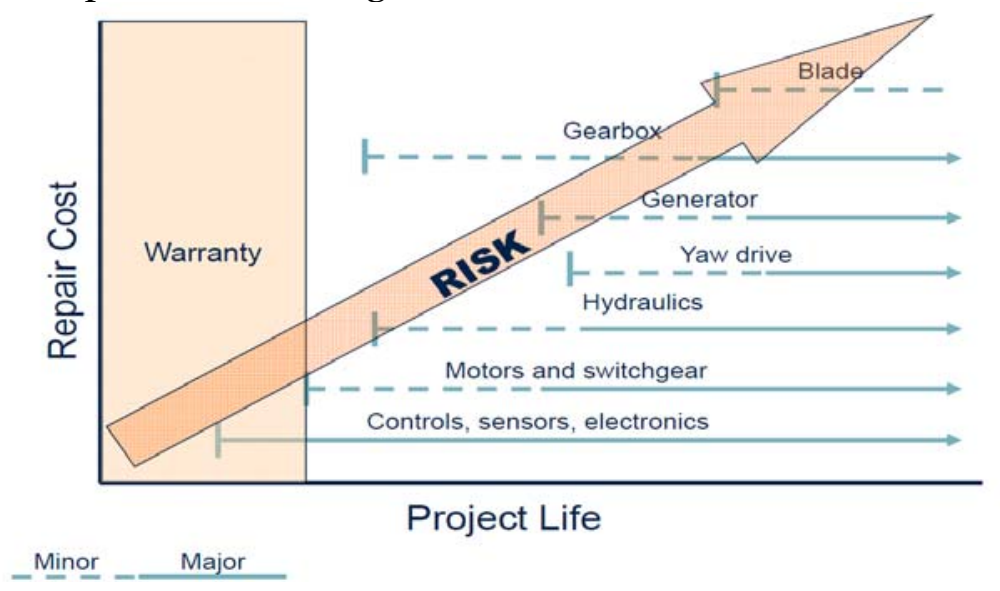

\subsection{IFOS's Solution}

IFOS, in collaboration with its STTR partner, Auburn University (AU), proposed a fiber Bragg grating (FBG) -integrated sensor system capable of providing real time in-situ defect detection, location and quantification of damage. In addition, the proposed system was demonstrated to be capable of validating wind turbine blade structural models, using recent advances in finite element analysis/modeling and non-destructive dynamic testing of composite structures. New generation Fiber-Optic methods will make it possible to analyze wind turbine blades not only non-destructively, but also without implanting intrusive electrical elements and transducers into the structure. The project's aim was to develop and implement FBGs as dynamic sensors attached to or embedded into the blades structure for damage assessment purposes. FBG sensors are capable of simultaneously measuring strain, vibration, pressure or temperature, parameters that can be used to monitor damage over wide temperature ranges. The IFOS proprietary FBG interrogation technique has been used to capture a wide-band dynamic response of the structure-under-test with high-sensitivity. Advanced signal processing and damage identification algorithms will also be applied to extract data with high accuracy and reliability. The IFOS solution could potentially evolve into an autonomous integrated monitoring system that can be used to inspect and perform Non-Destructive Evaluation (NDE).

In dealing with complex inspection problems, the flexibility of optical techniques and systems offers significant advantages for testing composite materials, ceramics, and liquids. The remote nature of optics based techniques is inherently compatible with rapid inspection of curved 


\section{Intelligent Fiber Optic Systems Corp. (IFOS) DOE STTR GRANT NO.: DE- SC0001020 \\ Interim Report \#4}

surfaces on either fixed or moving parts. Thus, optical methods, and, in particular, fiber-optic based systems and instruments can measure a large variety of components even in hostile environments or at temperatures well above those that can be tolerated using more conventional, contact-based techniques. The demonstrated accuracy and flexibility of fiber-optic based diagnostics systems led to attractive new sensing options in the non-destructive testing market. Optical sensor systems advantages include insensitivity to electromagnetic interference, freedom from sparking electrostatic discharge and light weight. In particular, fiber-optic sensing is typically applied to the structures being tested through a flexible harness, resulting in significant mass savings, flexible sensor distribution throughout structure, efficient multiplexing for high sensor capacity, low power requirements, high signal to noise ratio for high measurement accuracy, multi-parameter sensing and excellent potential for embedding in composite structures.

IFOS has been investigating fiber optic sensors for many years, embedding FBG sensors into a variety of structures for health monitoring. In past projects, IFOS has developed hardware, models and algorithms to detect and predict structural defects and failures. In this project, the proposed solution was specifically applied to (1) model-based blade structural performance monitoring; (2) high speed FBG sensors interrogation for structural-dynamic/shock wave responses measurement; and (3) detecting, locating and quantifying defects and degradations in wind turbine blade structure. Background and Technical Approach

\subsubsection{Fiber Bragg Grating (FBG) Sensors}

FBGs have proven useful, durable and cost effective for applications in telecommunication, civil and aerospace vehicles, oil exploration, mining machinery, and security. In the following sections their principle of operation are briefly described.

\section{FBG Sensor Principle of Operation}

The basic principle of an FBG-based sensor system involves monitoring of the wavelength shift of the returned Bragg-signal, as a function of the measurands (e.g. strain and temperature). The Bragg wavelength is related to the refractive index of the material and the grating pitch. Sensor systems that include such gratings work by injecting light from a spectrally broadband source into the fiber, with the grating either reflecting a narrow spectral component at the Bragg wavelength, or in transmission, this component is missing from the observed spectrum (see Figure 2). 

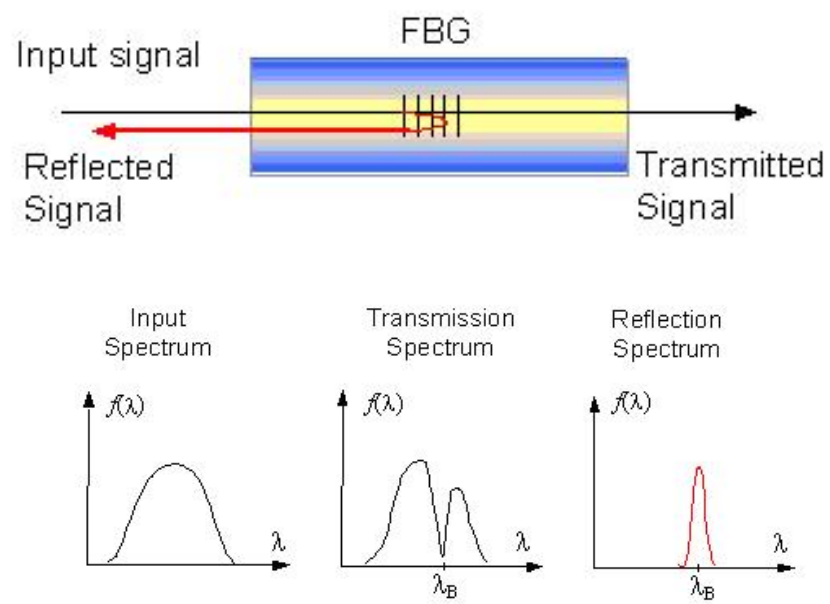

\section{Figure 2: $\quad$ Functional principle of a fiber optic Bragg grating}

Bragg gratings thus operate by acting as a wavelength selective filter that reflects a single wavelength, called the Bragg wavelength, $\lambda_{\mathrm{B}}$. The Bragg wavelength is related to the grating pitch, $\Lambda$, and the mean refractive index of the core, $\mathrm{n}$, by $\lambda_{\mathrm{B}}=2 \Lambda \mathrm{n}$. Both the fiber refractive index $(n)$ and the grating pitch $(\Lambda)$ vary with changes in strain $(\varepsilon)$ and temperature $(\Delta T)$, such that the Bragg wavelength shifts in response to longitudinal deformations in response to mechanical or thermal effects. This means that FBGs can be used as sensing elements. In a FBG sensor, the measurand causes a shift in the Bragg wavelength, $\Delta \lambda_{\mathrm{B}}$. The relative shifts in the Bragg wavelengths due to an applied strain $(\varepsilon)$ and a change in temperature $(\Delta T)$ are approximately given by the relationships

$$
\Delta \lambda_{B S}=\lambda_{B}\left(1-\rho_{\alpha}\right) \Delta \varepsilon
$$

$$
\Delta \lambda_{B T}=\lambda_{B}(\alpha+\xi) \Delta T
$$

where $\Delta \lambda_{\mathrm{BS}}$ and $\Delta \lambda_{\mathrm{BT}}$ are the strain and temperature induced Bragg wavelength shifts, $\lambda_{\mathrm{B}}$ is the Bragg wavelength, $\rho_{\alpha}$ is the photoelastic coefficient of the fiber $(\sim 0.22), \alpha$ is the coefficient of thermal expansion and $\xi$ is the thermo-optic coefficient. Operation as a sensor relies upon the measurement of the measurand induced shift in the Bragg wavelength. For FBGs written with Bragg wavelengths at $1550 \mathrm{~nm}$, the sensitivities to strain and temperature, measured at room temperature, are $\sim 1.2 \mathrm{pm} /\left(\mu\right.$ strain) and $\sim 10 \mathrm{pm} /{ }^{\circ} \mathrm{C}$ respectively.

The dynamic response of FBGs needs to be taken into account for their effectiveness in high bandwidth monitoring applications. The longitudinal resonance frequency of an FBG, $\omega$, is given by Eqn. 2 (approximately) as follows:

$$
\omega^{2}=\mathrm{E} /(\mathrm{L} . \mathrm{m})
$$

where $\mathrm{E}$ is the optical fiber Young's Modulus, $\mathrm{L}$ is the free length of fiber and $\mathrm{m}$ is the fiber mass density. Therefore, the resonance frequency of an FBG sensor of $5 \mathrm{~mm}$ length would be approximately $168 \mathrm{kHz}$. This particular sensor would be useful in measuring excitations with 


\section{Intelligent Fiber Optic Systems Corp. (IFOS) DOE STTR GRANT NO.: DE- SC0001020 \\ Interim Report \#4}

frequency content of up to $160 \mathrm{kHz}$. Using an optical fiber to sense external and unpredictable stresses/strain placed on composite structures has several advantages. First, the optical fiber transmission line is inherently immune to electromagnetic interference. Second, many kinds of sensing and/or controlling signals can be transmitted through a fiber, and power can be supplied through the same fiber in some cases. In addition, the sensitivity of the fiber sensors is excellent.

\subsubsection{Composite Materials with Embedded FBG Sensors}

The increased use of fiber reinforced composite materials is warranted due to their high strength/stiffness, low density, resistance to fatigue and corrosion, and their ability to be customized. The method of measurement is through monitoring of the strains measured by the embedded FBG sensors, followed by calculation of impact and damage at the points of interest. The Finite Element Model (FEM) of the corresponding composite structure was developed at Auburn University as part of the Phase I work to serve as the key analytical tool to be used for characterizing the blade structure using static testing.

\subsubsection{FBG-Sensor Interrogator}

The FBG sensor interrogator module is composed of an optical interface port for connecting to the multiplexed FBG sensors, sensor-specific front-ends modifiers, a parallel-architecture Photonic Spectral Processor (PSP) engine with customized Array Waveguide Gratings (AWGs), micro controllers and wired/wireless network interfaces. The schematic is shown in Figure 3. In this system, the PSP interacts with a broadband light source that sends light to the FBG sensors. The sensors reflect light with varying wavelengths depending upon the measurands (strain, temperature, acceleration, etc.) The PSP then processes the returned light that is then converted to electrical signals and then is processed by the electronics signal processing subsystem and finally interpreted by the algorithmic software subsystem.

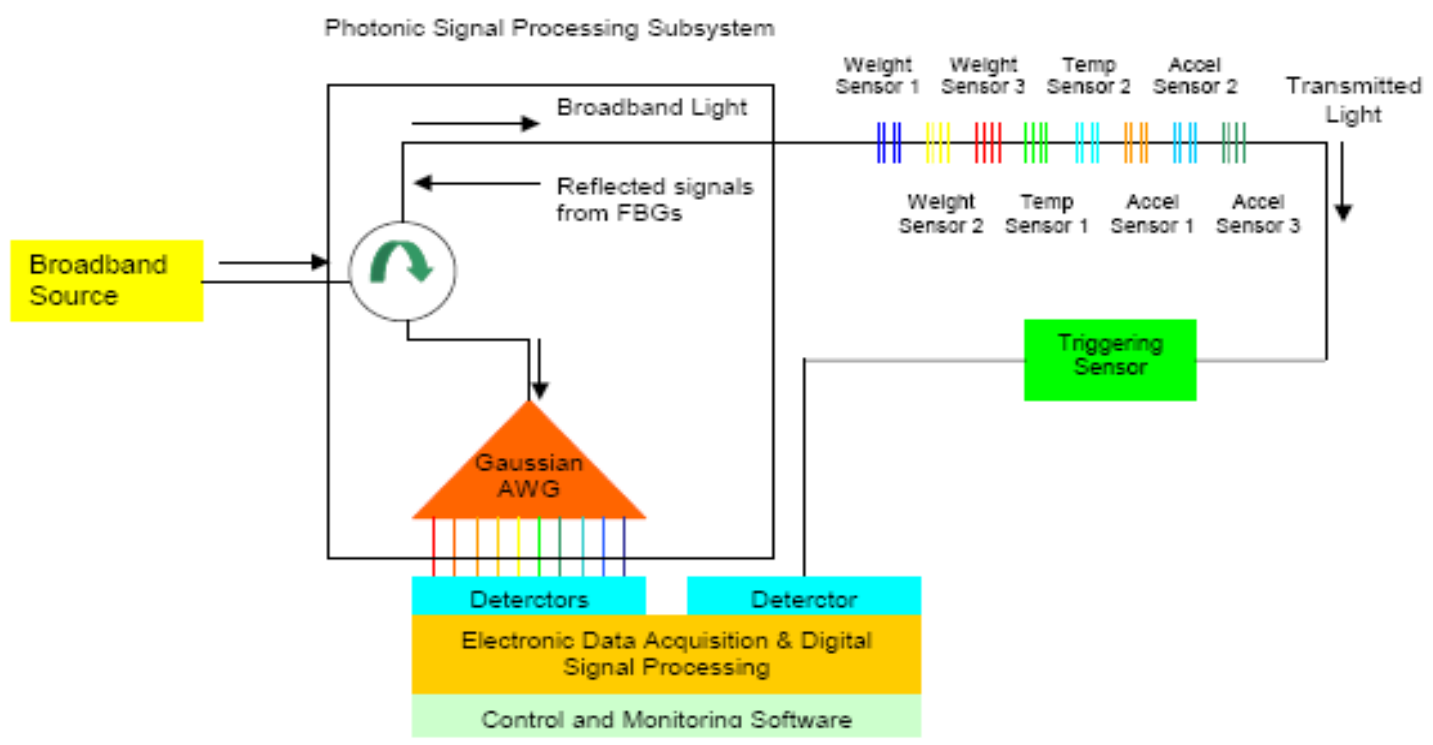

Figure 3: $\quad$ Schematic of FBG sensor interrogation system 


\section{Intelligent Fiber Optic Systems Corp. (IFOS) DOE STTR GRANT No.: DE- SC0001020 \\ Interim Report \#4}

The Phase I FBG-sensor interrogation system was customized for inspection and damage detection applications. Future enhancements will include, but not limited to: upgrades in portability, performance, intelligence and communication capability. Some system optimization will need to take place to achieve effective prototyping for the wind turbine blade application. It is envisioned that the system will ultimately be much smaller in size and include enabling fiber sensor networks as a plug-and-play unit to connect to the digital network world. The platform design of embedded electronics, wired and wireless transceivers, firmware, and software are capable of supporting network communications, control and multi-sensor processing.

\subsubsection{Competitive Advantages of the IFOS Approach}

Traditionally, sensors used to detect strain in structures involved electronic (e.g., resistive foil or piezoelectric) gauges, etc. However, these are susceptible to electromagnetic interference and lightning strikes, and do not have the very wideband frequency response and multiplexibility offered by the IFOS FBG approach.

Alternative FBG-based systems have been used for structural health monitoring. For example, Time Domain Reflectometry (TDR) has been used to allow monitoring of larger number of FBGs - at some cost to speed. Other approaches employ a swept laser technique which has speed limitations. The PSP technology developed by IFOS allows performing simultaneous measurements across a wide spectrum, which translates to unique advantage allowing high processing speed while interrogating a large number of FBGs with detection from quasi static strains to dynamic strains of $100 \mathrm{~s}$ of $\mathrm{kHz}$, using a single interrogator.

\subsubsection{Related Research Work Conducted by the Project Team}

\subsubsection{Work related to modeling of geometry and mechanics of PMC materials}

Many models used to calculate the elastic properties of textile composite materials from properties of their constituents are available in literature [for example, Pastore, 1994; Woo, 1997; Tarnopolski, 1973; Murthy, 1999] varying from closed-form to numerical solutions. Most of these models lack the detailed geometry that can represent the composites internal fabric architecture which may have a strong impact on the mechanical behavior of the fabric composite. Quantification of elastic properties requires an understanding of the spatial location of yarns and the contribution of each composite constituent to the composite overall response. To this end, a geometric model was developed by Professor Gowayed of the AU STTR Research Institute to evaluate the spatial location of yarns [Pastore, 1989] for CMC (ceramic matrix composite) and PMC (polymer matrix composite) materials. This mathematical model was able to model many 2D and 3D fabrics such as those shown in Figure 4.

The knowledge of yarn location and matrix distribution provide basis for application of numerical mechanical models, such as traditional finite element analysis (FEA), to evaluate the composite properties. Nevertheless, the use of traditional FEA is limited by the complexity of the fabric geometry and associated meshing problems, and requires a large number of elements. A typical plain weave fabric would require a few thousands elements to model [Woo, 1997]. It is expected that other complex fabrics, such as 3D fabrics, would require hundreds of thousands of elements. To solve this problem a hybrid FEA was developed by one of the authors [Gowayed, 1996] where a unit cell of the fabric architecture is identified and divided into hexahedra brick 


\section{Intelligent Fiber Optic Sy stems Corp. (IFOS) DOE STTR GRANT No.: DE- SC0001020 \\ Interim Report \#4}

elements with fiber and matrix around each integration point. Material homogenization was carried out around each integration point to define the anisotropic material response [Timoshenko, 1934]. The boundary conditions of the unit cell, dictated by the assumptions of repeatability and continuity, helped reduce the size of the stiffness matrix. A virtual work technique was used to calculate the elastic properties of the unit cell. Both geometric and mechanical FEA models were combined and integrated using a visual $\mathrm{C}++$ computer algorithm. This algorithm (pcGINA ${ }^{\odot}$ ) was utilized to support many composite programs and is currently distributed by the Air Force Research Laboratory, AFRL/RXLM, Wright-Patterson AFB, OH.
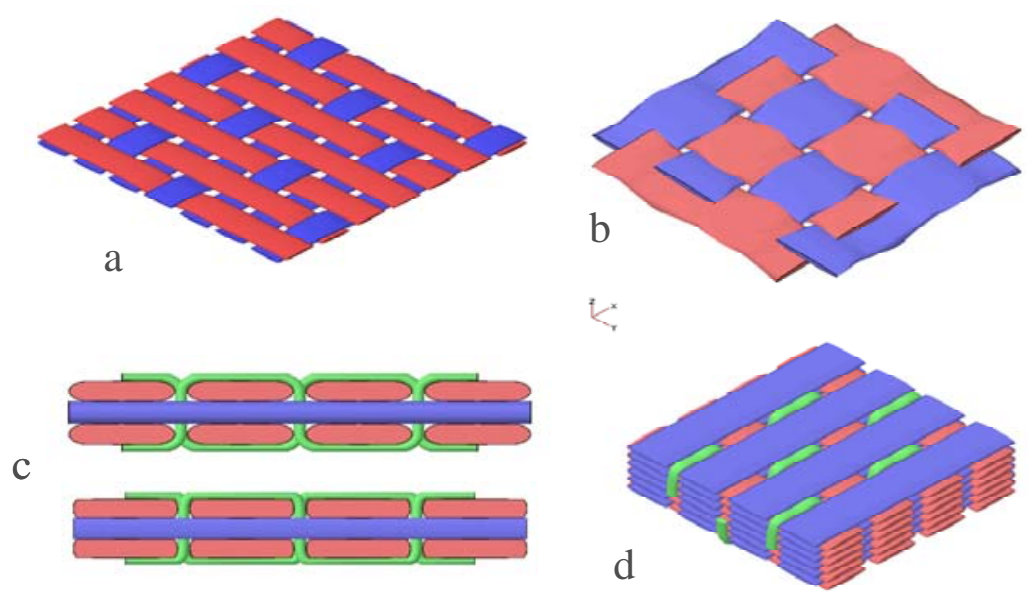

Figure 4: $\quad$ Graphic representation of geometric model using pcGINA ${ }^{\odot}$ of a) $5 \mathrm{HS}$, b) compressed plain weave, c) cross section view of a 3 layers $X Y Z$ orthogonal weave and d) 9 layers $X Y Z$ orthogonal weave.

\subsubsection{Research Work Available in Archived Literature}

\subsubsection{Dynamic Characterization}

Damping is one important parameter related to the study of dynamic behavior of composite structures in general. A sandwich composite (or "sandwich panel") is a structure consisting of two thin faces bonded to a thick lightweight core [Hornig, 2005; Nilsson, 2000]. The materials used to construct the faces are usually aluminum or a composite laminate, and the core can be a lightweight foam or a honeycomb structure of some other material. Chandra et al [Chandra, 1999] provided an extensive review of damping mechanisms in composites, key elements of which are detailed below:

- Viscoelastic nature of matrix and/ or fiber materials: the matrix shows the most significant contribution to the damping. In the case of high damping fibers, such as in carbon and Kevlar $^{\circledR}$ composites, the effect of the fibers damping should be included in the analysis.

- Damping due to interfaces: the interfaces are the regions where adjacent surfaces exist. In fiber-reinforced composites, the interfaces are the areas where the fibers are in direct contact with the matrix. In sandwich composites, on the other hand, the interfaces are the areas where the different layers are in contact with one other. 


\section{Intelligent Fiber Optic Sy stems Corp. (IFOS) DOE STTR GRANT No.: DE- SC0001020 \\ Interim Report \#4}

- Damping due to damage: this may be caused by frictional damping due to slip in the unbound regions between the fiber/matrix interface, and may also be caused by delaminations, and damping due to energy dissipation in the area of matrix cracks, broken fibers, etc. Damping effects due to damage will alter the vibration signature which is a critical health monitoring tool.

There are also other mechanisms of damping in composites, including viscoplastic and thermoelastic damping, which depend on the nonlinearities seen for high amplitude vibration levels, and on the cyclic heat flow from regions of compressive to tensile stresses, respectively.

Several damping measurement techniques have been developed, and the applicability of each of these methods is normally dependent on the frequency range of interest, and whether or not the damping in a resonant frequency is to be measured. Among the several approaches available for damping measurement, the half power method, logarithmic decrement, and hysteresis loops analysis are generally the most commonly used.

A variety of novel ideas for damping measurement has been introduced by several researchers, who have also described the critical factors for specific problems. Hooker [Hooker, 1981], for example, explains how damping measurements for biaxial hysteresis loops yield apparent loss factors, which may be related to the true loss factors of the system. On the other hand, So et al [So, 1990] describe a method for frequency-response-based damping measurement using a periodic chirp excitation, similar to pseudo-random noise excitation, but with a much higher peak-to-rms ratio. As well, McDaniel, Dupont, and Salvino [McDaniel, 200o] introduce an approach for the determination of a frequency-dependent damping from transient loadings.

\subsubsection{Condition Monitoring}

Vibration measurements have been used widely in machinery condition monitoring. In the 1990's, a number of researchers reported experimental investigations of incipient failure and dynamic behaviors. For example, Simpson et al. utilized ultrasound to test the energy storage flywheels that were fabricated with S2 glass-epoxy composites [Simpson, 1990]. This experiment was designed to study changes in the ultrasonic properties as a function of strain history and identified possible predictors of incipient failure. Tension specimens of the flywheel material were loaded uniaxially, and the ultrasonic properties (the shear and longitudinal wave velocities and the attenuation) were measured as a function of strain. The velocities were found to be excellent indicators of the maximum strain incurred at each point of the flywheel, and the attenuation delineates the region in which the stress is high enough to initiate micro cracking in the matrix.

In another work, Zheng et al. (1997) tested the input-output stability of the cracked rotor system [Zheng, 1997]. It was suggested that fatigue crack is an important reason for the existence of low frequency vibration components and a small fatigue crack may drive a system to instability in a very short time. Wu and Huang (1998) evaluated crack flexibility for a shaft-disk rotor with crack energy released [Wu, 1998]. From the FFT analysis of the displacement responses, the second harmonic component was extracted and served as a good index to detect the crack properties. Bachschmid et al. (2000), carried a model-based diagnostic approach to identify a transverse cracked rotor vibrations with $1 \mathrm{X}, 2 \mathrm{X}$ and $3 \mathrm{X}$ rev. components [Bachschmid, 2000]. 


\section{Intelligent Fiber Optic Systems Corp. (IFOS) DOE STTR GRANT No.: DE- SC0001020 \\ Interim Report \#4}

Darpe et al. (2003) investigated the steady state response of a cracked rotor experimentally [Darpe, 2003]. They proposed that the response of the rotor to axial impulse excitation could be used for diagnosis of rotor cracks, because spectral response of the cracked rotor with and without axial excitation is found to be distinctively different. Also, in their paper, it was shown that the spectral responses to periodic multiple axial impulses were present in the bending natural frequency as well as in the side bands around impulse excitation frequency and its harmonics. In addition, Green and Casey (2003) investigated the dynamics of a rotating shaft with transverse cracks extensively [Green, 2003]. The second harmonic component of the system response was shown to be the primary response characteristic resulting from the introduction of a crack.

\section{Anticipated Public Benefits}

The use of wind power capacity is expected to triple in the next five years worldwide. Around $194 \mathrm{GW}$ will be added between now and the end of 2014 to bring the grand total to $288 \mathrm{GW}$. The US added more wind power than any other country in 2007 [Rajgor 2008]. Currently, wind power constitutes $60 \%$ of renewable energy sales in the US vs. $40 \%$ for all other "green power" sources including biomass (includes ethanol), landfill gas, hydropower, geothermal and solar. As the public demand for and reliance on wind power grows the need for expansion, monitoring and preventive maintenance of wind power infrastructure becomes more imminent.

\subsection{Public Benefits}

The IFOS solution offers numerous potential benefits to the general public, contributing to greatly improved safety features and lower lifecycle costs of wind farms - both land-based and off-shore. Most importantly, it is recognized that enhanced safety and reduced operational costs is critical to the acceleration of alternative, non-carbon based, environment-friendly energy sources as a primary benefit to the general public. Preventing a wind turbine shut down due to an avoidable accident will tremendously improve safety and the economics of maintenance and repair. Early defect detection followed by the timely repair or retrofit, can yield to substantial savings resulting from avoidance of catastrophic failures and the scheduling of maintenance across an entire wind farm as opposed to costly single repairs.

Secondly, the overall maintenance cost of the wind turbine system will be reduced. Unplanned wind turbine breaks and service interruptions will reduce the required expense budget and allow those funds to be utilized in other needed areas. Reducing system maintenance cost will contribute to keeping the cost of energy down to heat homes, power lights, and energize business, medical, industrial, and other machinery throughout the US, and international markets. Power produced will be cleaner and cheaper and the public will potentially enjoy a demand-adaptable, non-interrupted supply of energy. Improving wind turbine efficiency and economics has important implications for national and regional initiatives that aim to displace large portions of the United State's dependency on fossil fuel.

In addition to large turbine power generation cost reduction, the proposed solution is applicable to small local wind turbine systems. This will also reduce the target profile for security risk across the national power distribution grid. The economics of small turbines are still at 3x cost of large turbines, but this may change as the technology develops and matures. 
Intelligent Fiber Optic Sy stems Corp. (IFOS) DOE STTR GRANT NO.: DE-

SC0001020

Interim Report \#4

\subsection{Marketing these Benefits}

Current key trends in the wind turbine markets are described in the study "Global Wind Turbine Markets and Strategies, 2008-2020" [EER 2008] as follows:

- Competition among wind turbine OEMs is rapidly intensifying as growth extends to new regions, encouraging start-ups of new manufacturers while pushing leading suppliers to expand their sales and production globally.

- Turbine prices, and the costs of installation, have trended upward over the last four years after nearly a decade of cost reductions per megawatt of nameplate capacity.

- Multiple players moving on $2 \mathrm{MW}$ and above segment: Vestas and Enercon-pioneers in $2 \mathrm{MW}$ and larger turbines-are aiming to protect their share of this market. However, multiple proven machines from Gamesa, Siemens, Suzlon/REpower, Alstom/Ecotecnia and others are providing buyers more options including CMS.

- Component suppliers face new challenges to keep pace with turbine demand, calling for major production capacity investments in the multi-megawatt segment, as well as a focus on local supply in booming new markets while keeping costs competitive.

\section{Degree to which Phase I has Demonstrated Technical Feasibility}

\subsection{Purpose of Phase I Research}

In Phase I, IFOS in collaboration with AU aimed to demonstrate the feasibility of an FBGintegrated sensor system capable of providing real time in-situ defect detection, location and quantification of damage based on combining the sensor data with a finite element model (FEM) of the blade.

The ultimate aim for the proposed system is to demonstrate monitoring capability and validate wind turbine blade structural models, using recent advances in non-contact, non-destructive dynamic testing of composite structures. This new generation method makes it possible to analyze wind turbine blades not only non-destructively, but also without implanting intrusive electrical elements and transducers into the structure.

\subsection{Research Carried Out}

IFOS and collaborators performed research under 6 Phase I tasks (covering 9 months):

1. Design and implement FBG sensor network embedded within composite panel

2. Design, build, and characterize proof-of-concept FBG interrogator

3. Explore \& develop decision-aid algorithms

4. Perform preliminary system testing \& characterization

5. Develop field deployment and transition plans

6. Deliver reports \& visit sponsor

The project was scheduled to run through until April $19^{\text {th }}, 2010$. Note that while the official start-date was back-dated to July $20^{\text {th }}, 2009$, the actual work commenced on October $1^{\text {st }}, 2009$. 


\section{Intelligent Fiber Optic Systems Corp. (IFOS) DOE STTR GRANT NO.: DE- SC0001020 \\ Interim Report \#4}

\subsection{Research Findings or Results}

The Appendix provides the details of the Phase I activities. The major findings are summarized below.

\subsubsection{Phase I Findings by AU}

- The experimental program, performed by AU in this project, provided solid indications that FBG sensors can detect elastic response of the tested composite coupons with high accuracy. Under different static and dynamic cases of loading, strain measurements of FBG sensors were in close agreement with those of standard experimental devices, such as the video extensometer and laser vibrometer.

- Results of FEA models of tested coupons matched experimental results providing confidence in the material properties incorporated in the model.

- It was observed that the introduction of the defect in the composite coupon caused a change of natural frequency from $52.3 \mathrm{~Hz}$ to $56 \mathrm{~Hz}$. This demonstrates that changes in natural frequencies of the wind turbine blades are affected by the presence and degree of damage.

- A turbine wind blade was modeled using FEA. The model was used to propose possible locations for FBG sensors based on maps of strain distribution under three standard cases of loading.

\subsubsection{Phase I Tests at NREL}

On January $25^{\text {th }}, 2010$, the IFOS research team traveled to the NREL wind test facility in Golden, $\mathrm{CO}$, and participated in measuring the response of SNL's sensor blade CX-100 under both static and dynamic loading. Participants included: from IFOS, Dr. Vahid Sotoudeh (PI), and Aleks Plavsic, from Sandia National Lab, Mark Rumsey, and from NREL-NWTC: Scott Hughes, Mike Jenks, and Jeroen Van Dam.

The two objectives set for of the tests performed at NREL were to:

1) measure strains occurring on the sensor blade's surface in response to static and dynamic loads, and

2) detect and measure potential acoustic emission events due to sensor blade's defects.

The first objective was addressed using the Phase I FBG interrogator. Strains were measured and recorded for four different static load values. In addition, strain measurements were performed during fatigue testing where dynamic loads were applied to the blade by two hydraulic actuators. The results and the accompanying analysis are presented in the Appendix.

For the second objective, a higher-speed interrogator developed under a separate IFOS project was utilized. This objective was met, by collecting FBG sensor data at $500 \mathrm{kHz}$ for 16 seconds during the fatigue test. This data was processed for identification and characterization of acoustic emission events, as shown in the Appendix.

\subsubsection{Phase I FBG Interrogator Developments}

For this work, IFOS has been developing an FBG interrogator for wind turbine blade monitoring applications with basic functionality tested in Phase I. Operation of the IFOS parallel processing 


\section{Intelligent Fiber Optic Systems Corp. (IFOS) DOE STTR GRANT No.: DE- SC0001020 \\ Interim Report \#4}

FBG interrogation system depicted in Figure 5 is as follows. Broadband light is sent from the broadband optical source to the Routing and Isolation Module (RIM) which directs it to one or multiple arrays of FBGs. the transmission path includes suitable isolation to prevent back reflection of the light towards the source.

This isolation may be achieved with an isolator or a circulator. Routing the light to multiple fibers may be accomplished using a passive splitter (no speed limits) or a switch (the switching speed limits the viewing times for FBGs on all fibers).

Routing the outgoing light to the outgoing fibers and the back-reflected light from FBGs can proceed using a circulator or a passive 2x2 splitter (with source isolator). However, the latter option loses $6 \mathrm{~dB}$ if only 1 output array is required.

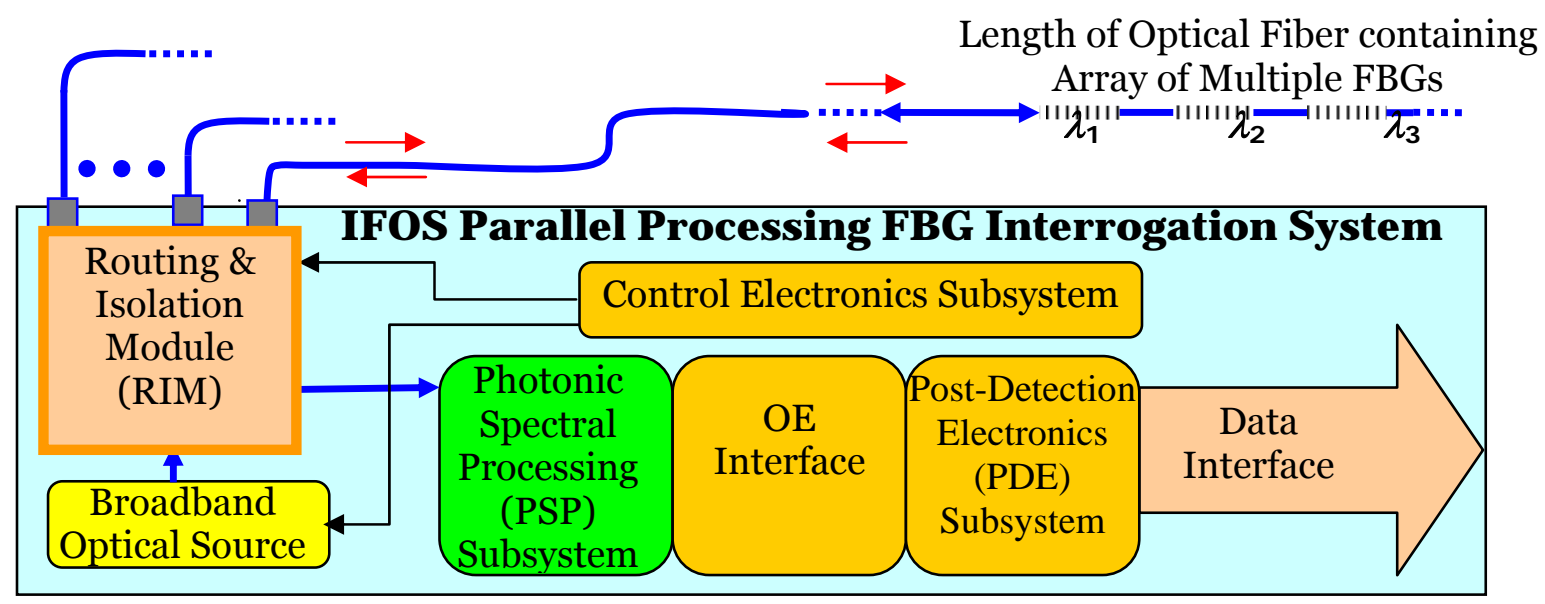

Figure 5: IFOS Parallel Processing FBG Sensor System Elements - Functional schematic showing FBG Sensors connected to a parallel processing FBG interrogation system comprising Broadband Optical Source, Routing \& Isolation Module (RIM), Photonic Spectral Processor (PSP), Post-Detection Electronics (PDE) and Data Interface.

The FBGs reflect wavelengths $\lambda_{1}, \lambda_{2}, \lambda_{3}$, etc., with each wavelength depending on the unstrained grating period for each particular FBG as well as the strain on that FBG. The reflected light passes back through the RIM which routes it to the Photonics Spectral Processing (PSP) subsystem. The PSP then divides it up into multiple channels and sends it through a multichannel optoelectronic interface and then on to a Post-Detection Electronics (PDE) subsystem followed by a data interface to a computer.

- For the Phase I interrogator

0 the PSP subsystem includes a standard narrow-band Gaussian AWG chip (48 channels spaced at $0.8 \mathrm{~nm}$ in the present implementation)

0 the PDE subsystem includes 48-channel trans-impedance amplifier (TIA) and analog-to-digital (A/D) functionality with $1.5 \mathrm{kHz}$ per FBG being the sampling rate (with the possibility of expanding to 64 or 128 channels). 
Intelligent Fiber Optic Sy stems Corp. (IFOS) DOE STTR GRANT NO.: DESC0001020

Interim Report \#4

\subsection{Estimate of Technical Feasibility}

The IFOS team clearly demonstrated the technical feasibility of an FBG-integrated sensor system capable of providing real time in-situ defect detection, location and quantification of damage, based on combining the sensor data with a finite element model (FEM) of the blade.

\subsection{Degree to which the Phase I Objectives have been Met}

Following are the Phase I objectives, each of which was successful met as also described below.

\section{Objective I-1: Conduct feasibility study. Down select potential designs}

This objective was successfully met by clearly showing the advantages of the selected fiber optic approach for the intended wind turbine blade application. We demonstrated that the FBG sensing approach was compatible with advanced wind turbine blade structures through modeling as well as installation of sensors in an SNL blade at NREL. We down-selected to an optimum combination of hardware for an FBG sensor interrogator by studying the design tradeoffs for meeting the key system performance parameter (accuracy, weight, cost, dynamic range, robustness) requirements. For example, for the interrogator, photonic signal processing using a diffraction grating signal processing was compared against an arrayed waveguide (AWG) approach and the latter found to be superior.

\section{Objective I-2: Develop proposed design for system}

IFOS and AU successfully developed a practical defect/damage system design consisting of (a) multiplexed FBG sensors and sensor interrogator, and (b) FEM for wind turbine blades.

\section{Objective I-3: Develop software capable of analyzing the data}

AU successfully developed and implemented practical defect/damage identification algorithms. The algorithm is designed to determine cumulative effects of stresses experienced as well as provide warning when component failure is likely. A FEM approach was developed for modeling the response of the composite blade structure response to both static and dynamic loading. Verification/validation steps were developed for the FEM to be used for base-line and defect/damage detection.

\section{Objective I-4: Build a model of the proposed system}

A Phase-I proof-of-concept model involving $1.5 \mathrm{kHz}$ FBG interrogator and multiple sensors was successfully developed.

\section{Objective I-5: Conduct proof-of-concept testing in a laboratory environment}

IFOS and AU conducted successful proof-of concept testing in house - See the Appendix for further details. 
Intelligent Fiber Optic Sy stems Corp. (IFOS) DOE STTR GRANT NO.: DE-

$\operatorname{SC0001020}$

Interim Report \#4

\section{Bibliography and References}

[Adewusi, 2001] Adewusi, S. A. and AL-Bedoor, B. O., "Wavelet Analysis of Vibration Signals of an Overhang Rotor with a Propagating Transverse Crack," Journal of Sound and Vibration, Vol. 246, No.5, pp. 777-793, 2001.

[Bachschmid, 2000] Bachschmid, N., Pennacchchi, P., Tanzi, E., and Vania, A., "Identification of Transverse Crack Position and Depth in Rotor Systems," Meccanica, Vol. 35, pp. 563-582, 2000.

[Bicego, 1999] Bicego, V., Lucon, E., Rinaldi, C., and Crudeli, R., "Failure Analysis of a Generator Rotor with a Deep Crack Detected During Operation: Fractorgraphic and Fracture Mechanics Approach," Nuclear Engineering and Design, Vol. 188, pp. 173-183, 1999.

[Chandra, 1999] Chandra, R., Singh, S.P., and Gupta, K. Damping Studies in FiberReinforced Composites - a Review, Composite Structures, 46(1), 41-51, (1999).

[Cusano, 2006] Cusano, A.; Capoluongo, P.; Campopiano, S.; et al. "Experimental modal analysis of an aircraft model wing by embedded fiber Bragg grating sensors," Sensors Journal, IEEE, Volume 6, Issue 1, 67 - 77 (2006).

[Darpe, 2003] Darpe, A. K., Gupta, K. and Chawla, A., "Experimental Investigations of the Response of a Cracked Rotor to Periodic Axial Excitation," Journal of Sound and Vibration, Vol. 260, pp. 265-286, 2003.

[Dawood, 2007] Dawood, T.A., Shenoi, R.A., Sahin, M. "A procedure to embed fibre Bragg grating strain sensors into GFRP sandwich structures," Composites Part A: Applied Science and Manufacturing, V38, Issue 1, 217-226 (2007).

[Green, 2003] Green, I., and Casey, C., "Crack Detection in a Rotor Dynamic System by Vibration Monitoring - Part I: Analysis," Proceedings of ASME Turbo Expro 2003, Atlanta, June 16-19, 2003.

[EER 2008] Emerging Energy Research, New Market Study, "Global Wind Turbine Markets and Strategies, 2008-2020", June 2008.

[Gentile, 2003] Gentile, A., and Messina, A., "On the Continuous Wavelet Transforms Applied to Discrete Vibrational Data for Detecting Open Cracks in Damaged Beams," Solids and Structures, Vol. 40, pp. 295-315, 2003.

[Gowayed, 1996] Gowayed Y, Pastore C., and Howarth C., "Modification and application of a unit cell continuum model to predict the elastic properties of textile composites," Composites Part A 1996; 27a(2):149.

[Hornig, 2005] Hornig, K.H., and Flowers, G.T. Parameter Characterisation of the Bouc/Wen Mechanical Hysteresis Model for Sandwich Composite Materials using Real Coded Genetic Algorithms, Intl. J. of Acoustics and Vibration. (IJAV), 10(2), 73-81, (2005).

[Hooker, 1981] Hooker, R.J. On the Interpretation of Biaxial Hysteresis Loops, J. of Sound and Vib., 76(3), 463-466, (1981).

IFOS Proprietary Fiber-Optic Defect \& Damage Locator System for Wind Turbine Blades 


\section{Intelligent Fiber Optic Systems Corp. (IFOS) DOE STTR GRANT NO.: DE- SC0001020 \\ Interim Report \#4}

[Li, 2004] Li, Z., Xia, S., Fan, J., and Su, X., "Damage Detection of Beams Based on Experimental Wavelet Analysis of Flexible Waves," Key Engineering Materials, v 261-263, No. II, Advances in Fracture and Failure Prevention: Proceedings of the Fifth International Conference on Fracture and Strength of Solids (FEOFS2003): Second International Conference on Physics and Chemistry, 2004.

[Liu, 1998] Liu, G., Wu, G.; Jia, D., Shangguan, J., Wang, Z. and Zhou, H., “Application of Gabor Transform for Detecting Crack Edge-reproduced Wave from Reactor Pressure Vessel," Hedongli Gongcheng/Nuclear Power Engineering, Vol. 19, No. 6, pp. 514-518, 1998.

[Kim, 2003] Kim, S. S. and Kim, J. H., "Rotating Composite Beam with a Breathing Crack," Composite Structures, Vol. 60, pp. 83-90, 2003.

[McDaniel, 200o] McDaniel, J.G., Dupont, P., and Salvino, L. A Wave Approach To Estimating Frequency-Dependent Damping Under Transient Loading, J. of Sound and Vib., 231(2), 433-449, (2000).

[Murthy, 1999] Murthy P, DiCarlo J, and Mital S., "Characterizing the properties of a 5-H woven $\mathrm{SiC} / \mathrm{SiC}$ composite using CEMCAN/WCMC computer code," 23rd annual conference on composite materials and structures (restricted sessions) 1999. Cocoa Beach, Florida.

[Nilsson, 2000] Nilsson, E. Some Dynamic Properties of Honeycomb Panels, Report, Royal Inst. of Tech., Stockholm, Sweden, (2000).

[Pastore, 1989] Pastore C, Gowayed Y, and Cai YJ "Application of computer aided geometric modeling for textile structural composites," CADCOM 1989, 45, Southampton, UK: Computational Mechanics Publications.

[Pastore, 1994] Pastore C and Gowayed Y, "A self-consistent fabric geometry model: modification and application of a fabric geometry model to predict the elastic properties of textile composites," Journal of Composite Technology and Research 1994;16 (1): 32-36.

[Persson, 1996] Persson, H.W., Hakansson, H. Remote vibration measurements using airborne ultrasound, Ultrasonics Symposium, 1996. Proceedings., Volume: 1, page(s): 689-692 vol.1

[Prabhugoud, 2006] Prabhugoud, M., Peters, K.,"Finite Element Model for Embedded Fiber Bragg Grating Sensors," Institute of Physics Publishing, Dirac House, Temple Back, Bristol BS1 6BE, UK, Smart Materials and Structures, volume 15, number 2, pages 550-562 (2006).

[Rajgor 2008] Rajgor, G., "Big Hike Upwards for Five Year Forecast,” Wind Power Monthly, May 2008, pp96-98

[Rumsey, 2008] Rumsey, M.A., "Sensor Projects at Sandia National Laboratories," 2008 Wind Turbine Blade Workshop, May 2008.

[Simpson, 1992] Simpson, W. A. Jr. and McClung, R. W., "Ultrasonic detection of fatigue damage in glass-epoxy composite flywheels", ASTM Special Technical 


\section{Intelligent Fiber Optic Systems Corp. (IFOS) DOE STTR GRANT No.: DE- \\ SC0001020 \\ Interim Report \#4}

Publication, International Symposium on Damage Detection and Quality Assurance in Composite Materials, Nov 13-14, 1992.

[So, 1990] So, C.K., Lai, T.C., and Tse, P.C. The Measurement of Material Damping by FreeVibration Technique with Periodic Excitation, Experimental Techniques, 14(3), 41-42, (1990).

[Takeda, 2005] Takeda, N., Okabe, Y., Kuwahara, J., Kojima, S., and Ogisu, T., 2005, Development of smart composite structures with small-diameter fiber Bragg grating sensors for damage detection: Quantitative evaluation of delamination length in CFRP laminates using Lamb wave sensing, Composites Science and Technology 65 2575-2587.

[Tanopolski, 1973] Tarnopolski YM, Polyakov VA and Zhigun IG. Polymer Mechanics 1973;(5): 853 .

[Thyagarajan, 1998] Thyagarajan, S. K., Schulz, M. J., Pai, P. F., and Chung, J., "Detecting Structural Damage using Frequency Response Functions," Journal of Sound and Vibration , 1998, Vol. 210, No. 1, pp. 162-170.

[Timoshenko, 1934] Timoshenko S.P., “Theory of Elasticity,” New York (1934).

[Vanladuit] S. Vanlanduit, a, , F. Daerdena and P. Guillaumea, Experimental modal testing using pressurized air excitation Department of Mechanical Engineering (MECH), Vrije Universiteit Brussel, Pleinlaan 2, B-1050 Brussels, Belgium

[Verbruggen 03] Verbruggen, T. W., "Wind Turbine Operation \& Maintenance Based on Condition Monitoring: WT-OMEGA," ECN(Energy research Center of the Netherlands) Final Report, Jan 2003

[Woo, 1997] Woo K, Whitcomb J, "Global/local finite element analysis for textile composites," Journal of Composite Materials 1997;28(14):1305-21.

[Wu, 1998] Wu, M. C. and Huang, S. C., "Vibration and Crack Detection of a Rotor with Speed-dependent Bearing”, International Journal of Mechanical Science, Vol. 40, No. 6, pp. 545-555, 1998.

[Zheng, 1997] Zheng, G.T., and Leung, A. Y., "On the Stability of Cracked Rotor Systems," Journal of the Chinese Society of Mechanical Engineering, Vol. 19, No. 1, pp. 125$133,1997$. 
Intelligent Fiber Optic Sy stems Corp. (IFOS) DOE STTR GRANT No.: DE-

SC0001020

Interim Report \#4

\section{Appendix: Phase I Result Details}

\subsection{Test Coupon Development, Sensorization and Testing}

At Auburn University, samples for tensile and four point bending tests were cut according to specifications from the composite plate. Edges were polished to remove the effect of cutting and ensure that no stress concentration will impact the results of the tests.

All samples (Figure 6) were mailed from Auburn to IFOS for evaluation and mounting of the FBG Sensors.

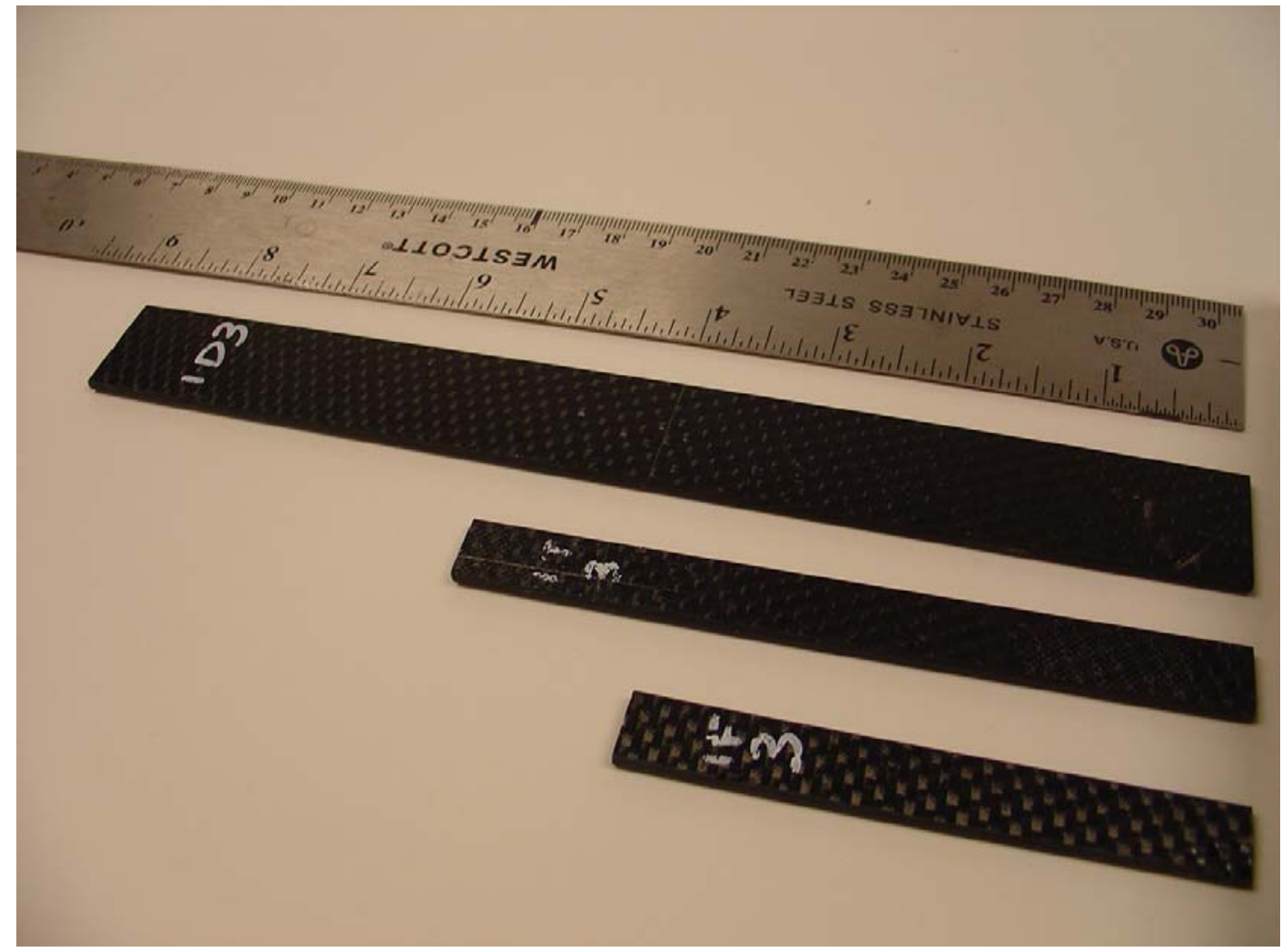

Figure 6: Three of the composite coupons

IFOS mounted FBG sensors on nine composite coupons (three of each of three different lengths) as shown in Figure 7. 


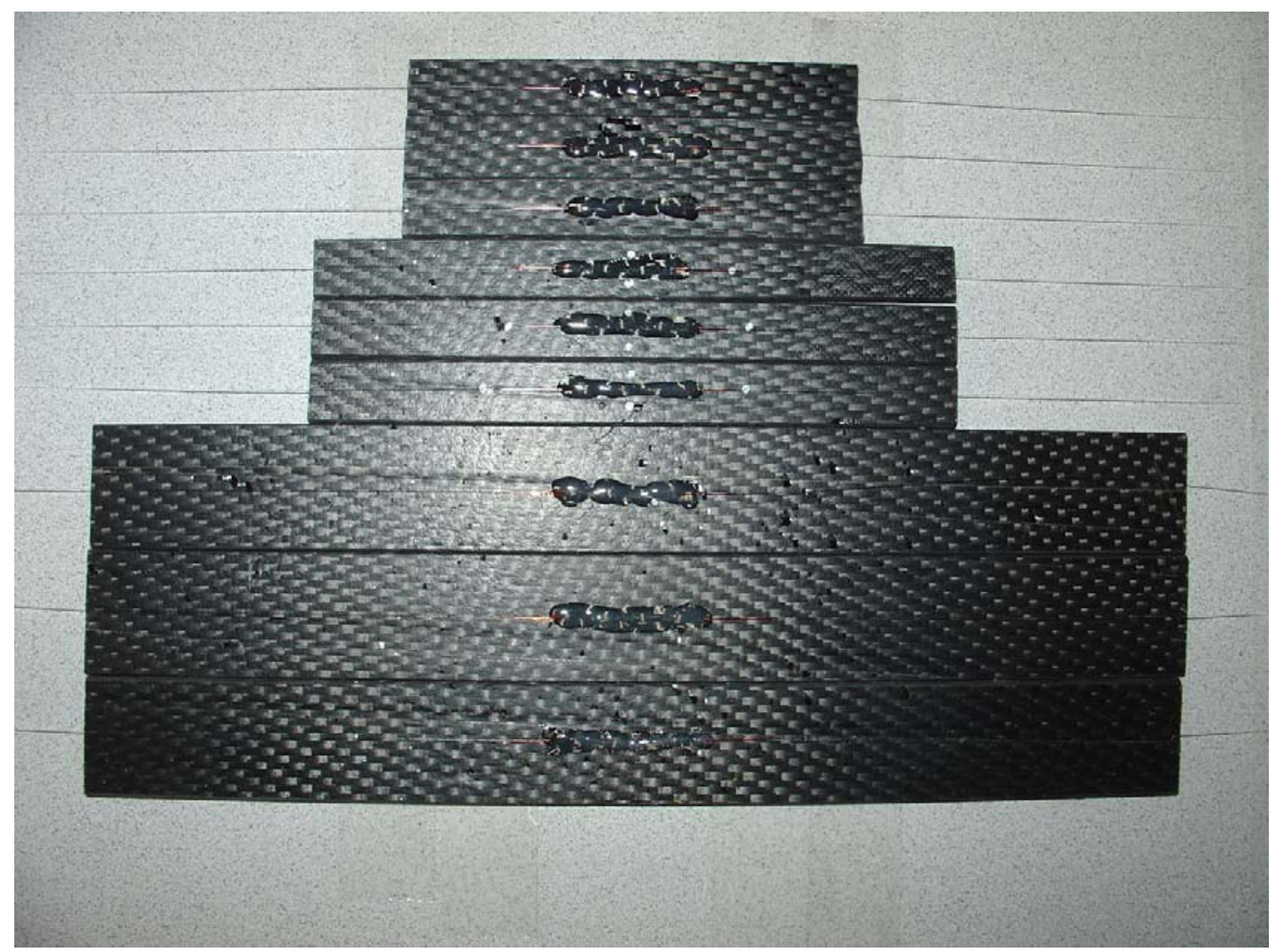

Figure 7: Composite coupons with surface mounted FBGs

Details of coupons testing and finite element modeling at Auburn University are shown in Appendix A.

\subsection{FBG Interrogation}

Figure 8 shows the IFOS parallel processing FBG interrogation design which IFOS was implementing at $1 \mathrm{kHz}$ for Phase I. In Phase II, IFOS will upgrade the design to a field installable system. In this system:

- The Broadband Optical Source Module (BOSS) sends light out via the Routing and Interface Module (RIM) to multiple arrays of FBG sensors.

- The sensors then return selected wavelengths depending upon the measurands (load, strain, weight, pressure, temperature, etc.) imposed upon them to the RIM which in turn routes it to the Photonic Spectral Processing (PSP) Subsystem.

- The PSP subsystem then processes the returned light which is then converted by the OE (optoelectronic) interface (photodetector array(s)) as multiple wavelengthdependent currents that are in turn processed by the Post-Detection Electronics (PDE) subsystem and sent as digital data through a data interface for final interpretation by the algorithmic software subsystem that can be either on-board the interrogator or in a central computer. 
Intelligent Fiber Optic Sy stems Corp. (IFOS) DOE STTR GRANT No.: DE-

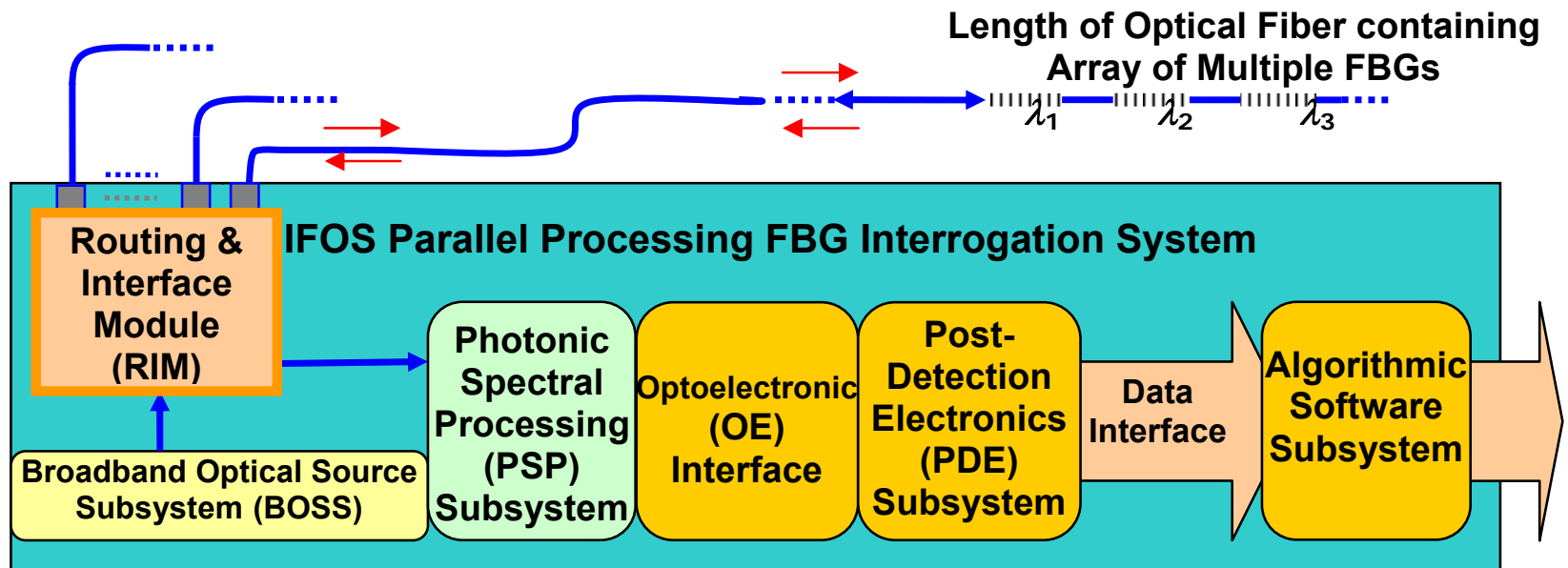

Figure 8: IFOS parallel processing FBG interrogation supporting multiple FBG arrays

\subsection{IFOS-Sandia-NREL Wind Turbine Blade Test at NWTC}

IFOS participated in testing of a wind turbine blade at NREL on January $25^{\text {th }}$ as described below. The mail goal of this effort was attaching IFOS FBG sensors to the CX-10o blade underdynamic-test at NREL, record the dynamic strain signals detected by the FBGs and recorded by the IFOS very high speed interrogator model at $500 \mathrm{kHz}$ and check for presence of acoustic emissions (AE) signals. This effort was taken by IFOS beyond the original Phase I scope of work.

\subsubsection{FBG Sensor Arrays}

Two arrays of three fiber Bragg grating (FBG) sensors were installed on the top (Array 1) and bottom (Array 2) of Sandia Sensor Blade (CX-100, blade \#011) between the anchored base of the blade and the main load-actuating saddle. After sanding off the epoxy-based paint at the installation locations, the sensors were epoxied to the composite high-pressure skin external surface of the blade. Table 1 shows the nominal wavelengths of the FBGs within each array. Figure 9 shows the installation locations of six FBGs (3 FBGs per array) on the test blade. IFOS Mini I*Sense model 48000 interrogator was used to measure the FBG sensors outputs. 
Intelligent Fiber Optic Sy stems Corp. (IFOS) DOE STTR GRANT No.: DE-

Table 1: FBG Sensor Arrays Specifications

\begin{tabular}{|l|c|l|c|c|c|c|}
\hline & \multicolumn{2}{|c|}{ FBG1 } & \multicolumn{2}{c|}{ FBG2 } & \multicolumn{2}{c|}{ FBG3 } \\
\hline Sensor Array No. & $\mathrm{nm}^{1)}$ & in $^{2}$ & $\mathrm{~nm}$ & in & nm & in \\
\hline 1 - Top & 1544.13 & 20 & 1547.98 & 39 & 1551.98 & 55 \\
\hline 2 - Bottom & 1544.16 & 20 & 1547.99 & 39 & 1552.02 & 55 \\
\hline Notes: \\
1) nominal wavelengths are provided by the FBG manufacturer \\
2) locations are measured from the anchored base of the blade \\
\hline
\end{tabular}

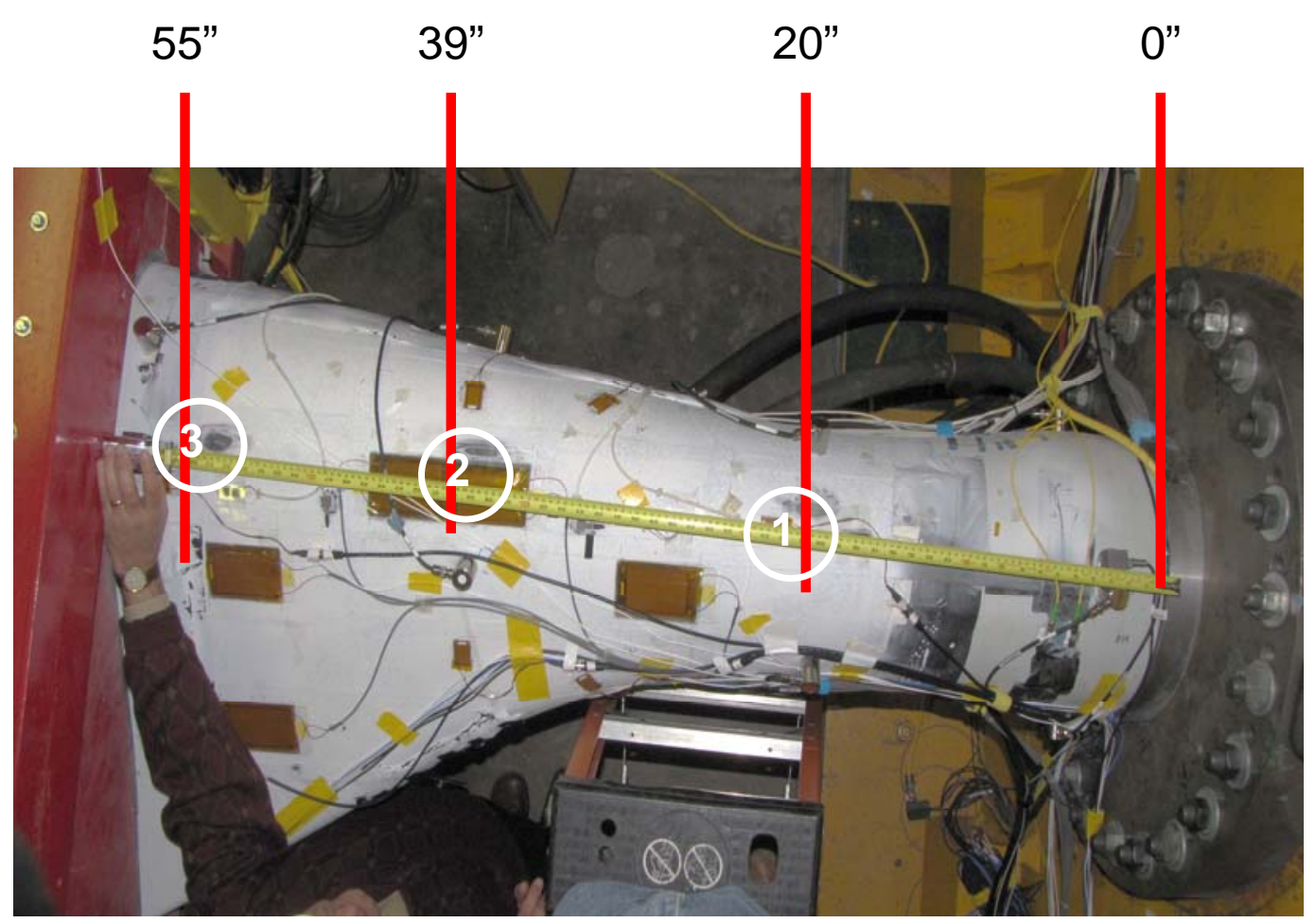

Figure 9: FBG Sensor Arrays Installation Locations

\subsubsection{Static Test}

After $70 \mathrm{~K}$ fatigue cycles were put on the Sensor Blade, the fatigue test was temporally stopped to perform a series of static tests. During the static test, four different vertical loads, at $50 \mathrm{lbs}$ increment, were applied at the outboard saddle position near the tip of the blade. Table 2 shows strain readings by each of the six FBG strain sensors at each static load level. 
Intelligent Fiber Optic Sy stems Corp. (IFOS) DOE STTR GRANT No.: DESC0001020

Interim Report \#4

Table 2: Static Test FBG Sensor Readings

\begin{tabular}{|c|c|c|c|c|c|c|c|c|}
\hline & & & & & ATIC & OAD & & \\
\hline \multirow{7}{*}{$\begin{array}{l}\text { STRAIN } \\
(\mu \varepsilon)\end{array}$} & Array \# & FBG \# & 0 (initial) & 50 & 100 & 150 & 200 & 0 (final) \\
\hline & \multirow{3}{*}{ 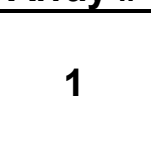 } & 1 & 48 & 71 & 93 & 113 & 132 & 36 \\
\hline & & 2 & -10 & 14 & 37 & 59 & 79 & -20 \\
\hline & & 3 & 27 & 56 & 83 & 109 & 132 & 12 \\
\hline & \multirow{3}{*}{2} & 1 & -11 & -24 & -37 & -47 & -57 & 0 \\
\hline & & 2 & 9 & -31 & -68 & -101 & -133 & 31 \\
\hline & & 3 & -28 & -48 & -67 & -82 & -95 & -11 \\
\hline
\end{tabular}

\subsubsection{Fatigue Test}

After the static test, the fatigue test of the sensor blade resumed where two hydraulic actuators mounted on each side of the main saddle applied sinusoidal load to the blade at $1.8 \mathrm{~Hz}$ in nearly vertical direction. Figure 10 shows the time histories of strain readings from Array 1 (Top) three FBGs. Time histories of strain readings from Array 2 (Bottom) three FBGs are shown in Figure 11. Minima and Maxima of strain readings are shown in Table 3. Information about maximal load values will be provided by Mark Rumsey from Sandia National Lab. The FBGs sensor arrays will remain in place for the duration of the blade fatigue test.

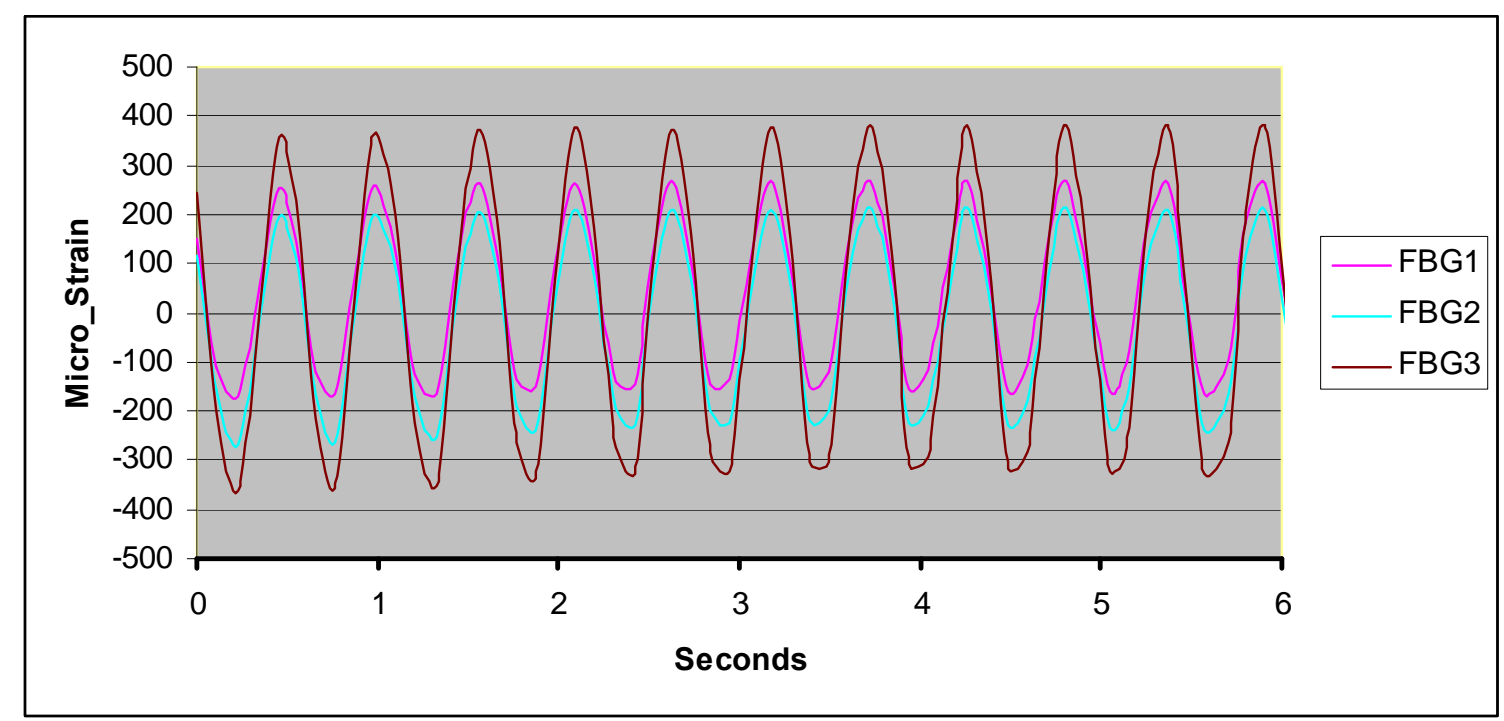

Figure 10: Fatigue test - Array 1 (Top) FBG Sensors Readings Time Histories 
Intelligent Fiber Optic Sy stems Corp. (IFOS) DOE STTR GRANT No.: DE-

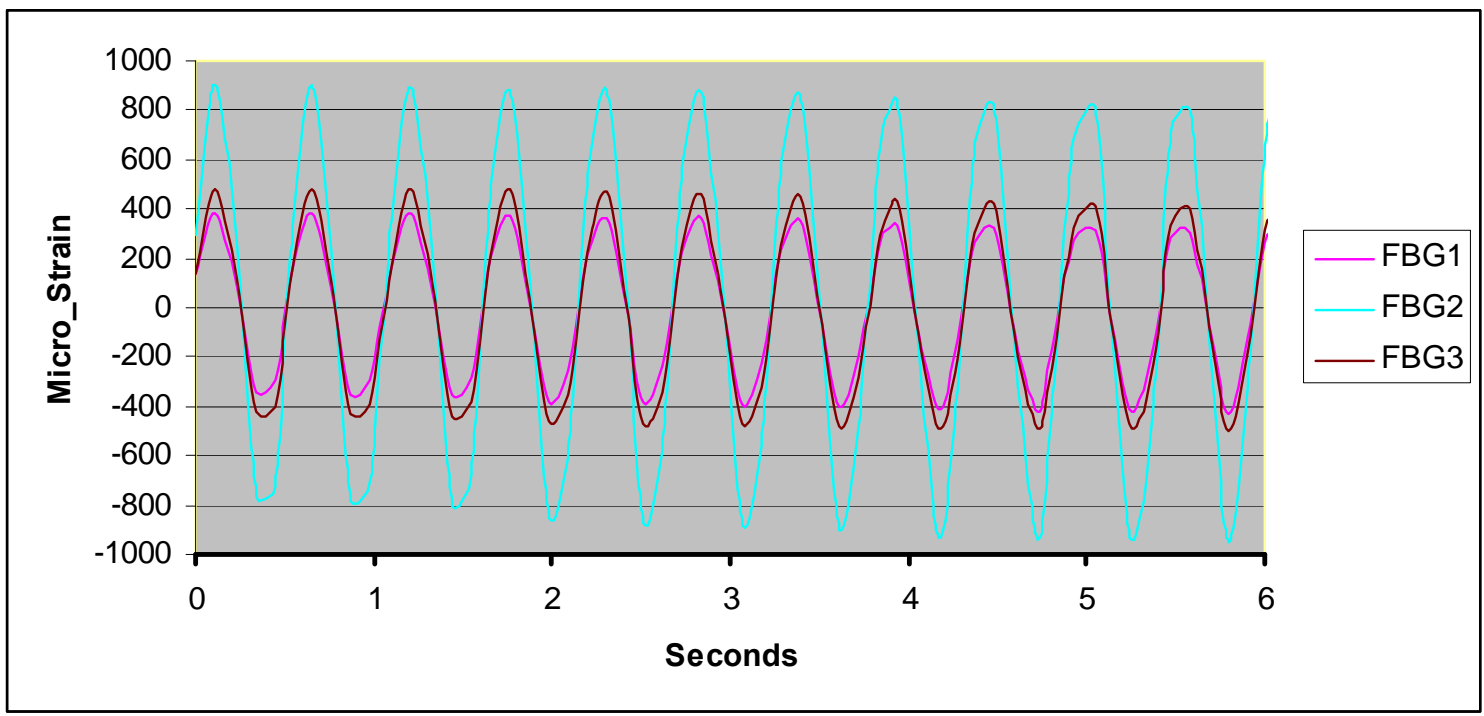

Figure 11: Fatigue test - Array 1 (Top) FBG Sensors Readings Time Histories

Table 3: Fatigue Test FBG Sensor Readings

\begin{tabular}{|c|c|c|c|}
\hline & & \multicolumn{2}{|c|}{ STRAIN $(\boldsymbol{\mu \varepsilon})$} \\
\hline Array \# & FBG \# & Maximal & Minimal \\
\hline \multirow{3}{*}{$\mathbf{1}$} & $\mathbf{1}$ & 269 & -187 \\
\cline { 2 - 4 } & $\mathbf{2}$ & 212 & -274 \\
\cline { 2 - 4 } & $\mathbf{3}$ & 383 & -366 \\
\hline \multirow{3}{*}{$\mathbf{2}$} & $\mathbf{1}$ & 385 & -427 \\
\cline { 2 - 4 } & $\mathbf{2}$ & 898 & -952 \\
\cline { 2 - 4 } & $\mathbf{3}$ & 485 & -499 \\
\hline
\end{tabular}

\subsubsection{Summary}

The two objectives of the test were 1) to measure strains occurring on the sensor blade's surface in response to static and dynamic loads and 2) to detect and measure potential acoustic emission events due to sensor blade's defects. The first objective was accomplished by using Mini I*Sense 48000 interrogator. Strains were measured and recorded for four different static load values and for dynamic loads produced by mechanical excitations of the blade (fatigue test). This data will be used by Auburn University for validation of the blade's finite element model. For the second objective, high-speed interrogator was used. This objective was partially accomplished by collecting FBG sensors' data at $500 \mathrm{kHz}$ for 16 seconds during the fatigue test. This data is currently being processed for identification and characterization of acoustic emission events, if any.

\subsubsection{Participants}

IFOS: Vahid Sotoudeh, Aleks Plavsic

Sandia National Lab: Mark Rumsey

NREL-NWTC: Scott Hughes, Mike Jenks, Jeroen Van Dam 
Intelligent Fiber Optic Sy stems Corp. (IFOS) DOE STTR GRANT No.: DE-

SC0001020

Interim Report \#4

\subsubsection{Equipment used}

- High speed wavelength interrogator $=I^{*}$ Sense $18000+500 \mathrm{kHz}$ DAQ

- 2 single fiber FBG arrays each with three FBGs

- Array \#1 was positioned on the top of the blade's base and array \# 2 was positioned on the bottom of the blade's base

- The FBGs were epoxied to composite skin of the blade after sanding off the paint at installation locations.

- The FBGs were separated by 15 to 20 inches.

\subsubsection{Procedure}

- Array \# 1 fiber was connected first to the front end of the high speed interrogator (I*Sense 1800o)

- The blade was mechanically excited by two hydraulic actuators which applied sinusoidal load to the blade at $1.8 \mathrm{~Hz}$ in nearly vertical direction

- Data was captured at $500 \mathrm{kHz}$ at 16 seconds intervals on all 16 channels (numbered o15)

- The strongest signals were observed on the DAQ channels 4, 9, and 14.

- The data collected on these three channels were post processed by using Matlab scripts

\subsubsection{Results}

NOTE1: Due to high processing times, high memory usage, and in order to have clearer plots, partial captured signals instead of full captured signals $(16 \mathrm{sec})$ are shown below. However, all the necessary information is contained in these partial plots.

NOTE2: Some plots of the original signals below do not show sinusoidal waveforms because the analog output of the High speed interrogator has not been linearized and calibrated.

\subsubsection{Array \#1 (Top)}

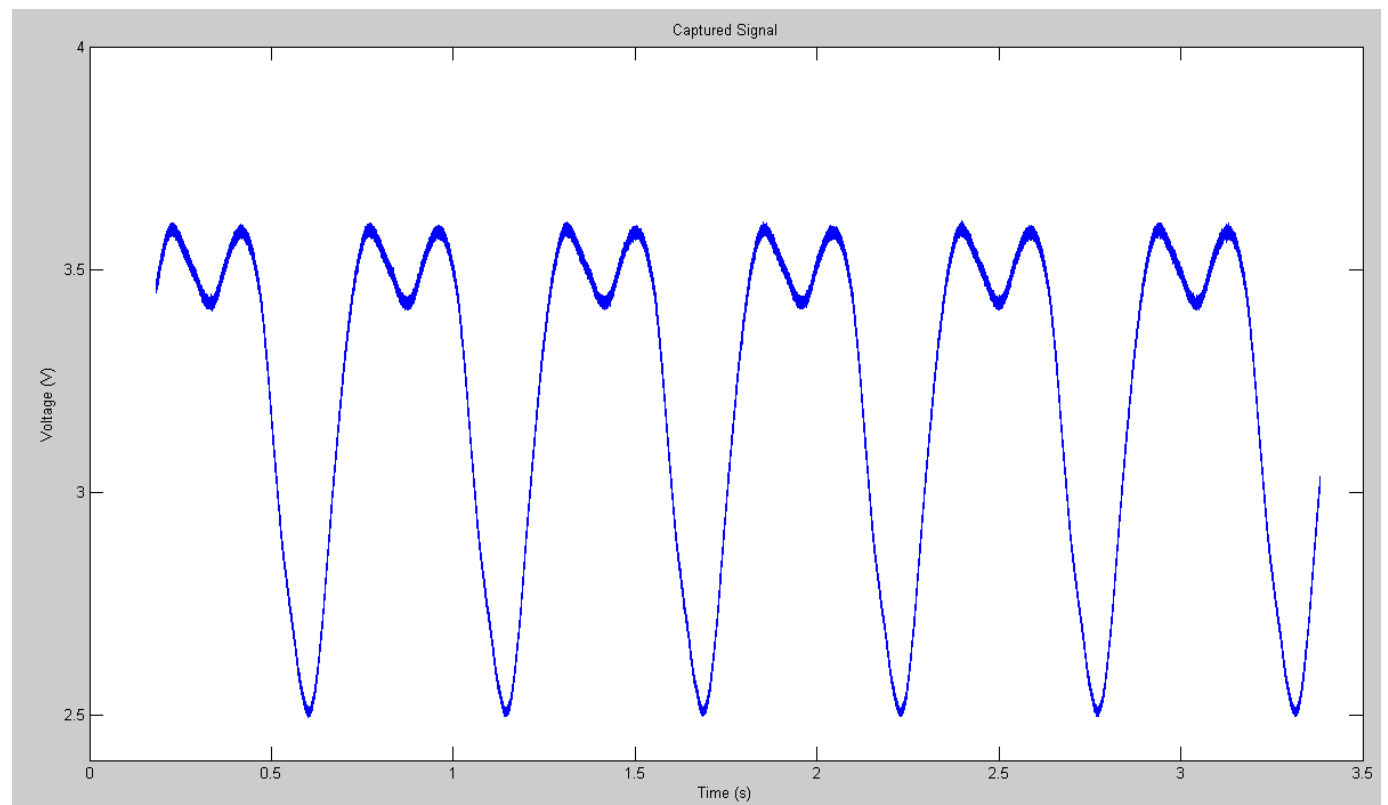

Figure 12: Array \#1 (top): Original signal from FBG \#1 (Ch. 9) 
Intelligent Fiber Optic Systems Corp. (IFOS) DOE STTR GRANT No.: DE-

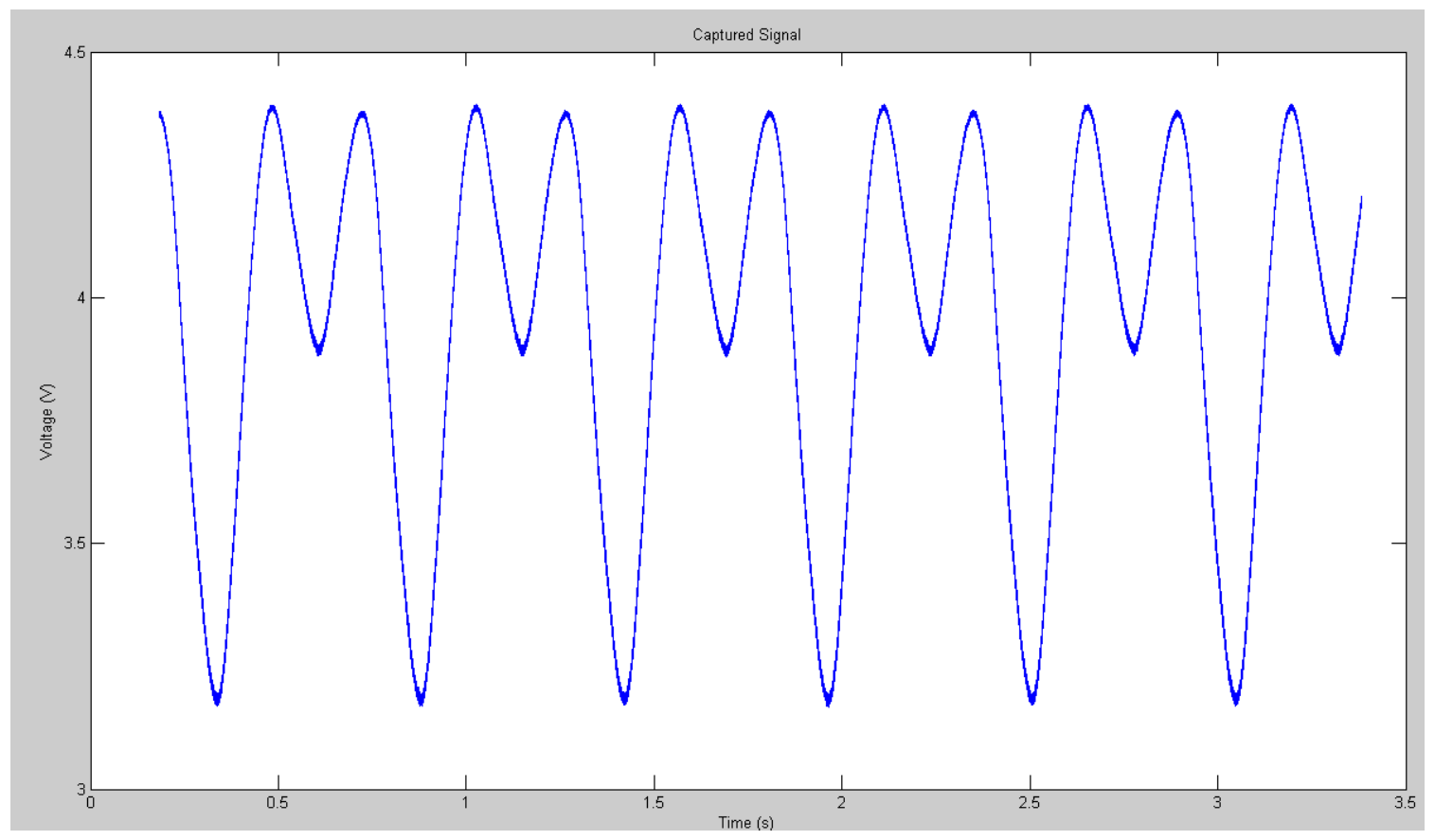

Figure 13: $\quad$ Array \#1 (top): Original signal from FBG \#2 (Ch. 4)

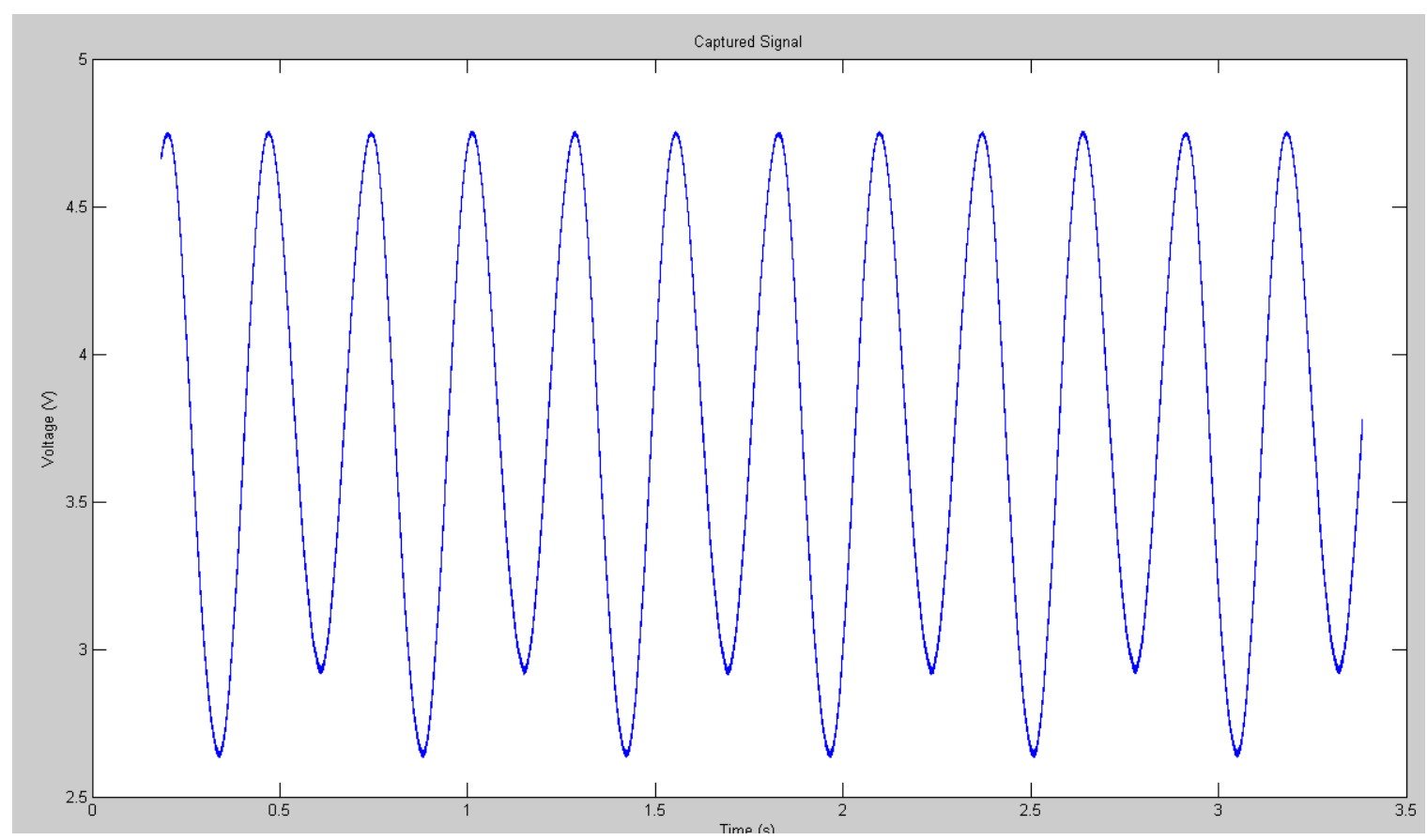

Figure 14: Array \#1 (top): Original signal from FBG \#3 (Ch. 14) 
Intelligent Fiber Optic Sy stems Corp. (IFOS) DOE STTR GRANT NO.: DE-

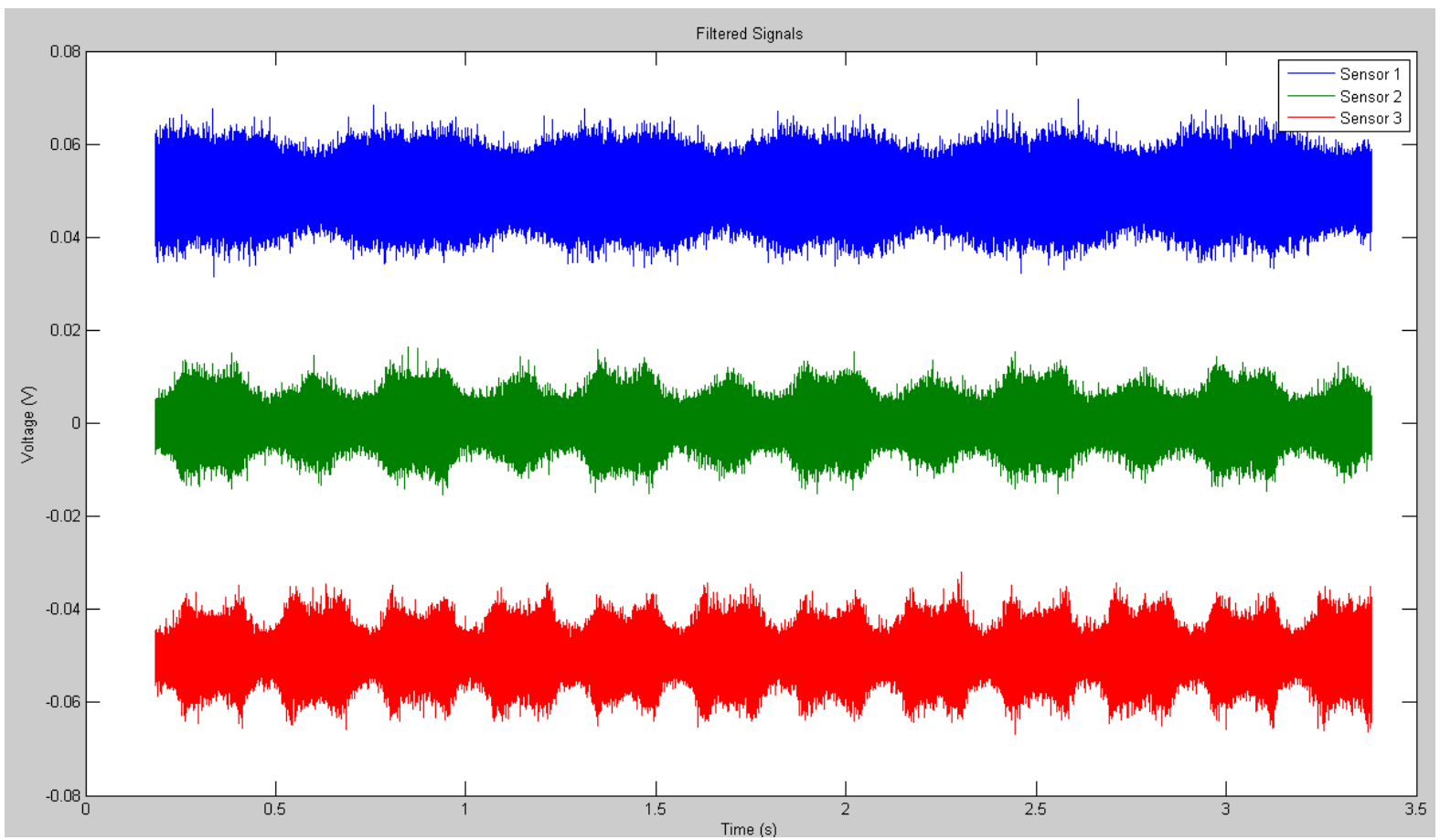

Figure 15: $\quad$ Array \#1 (top): Post processed signals (Ch 9 plot is offset by $0.05 \mathrm{~V}$ and $\mathrm{Ch}$ 14 plot is offset by $-0.05 \mathrm{~V}$ for a clearer view):

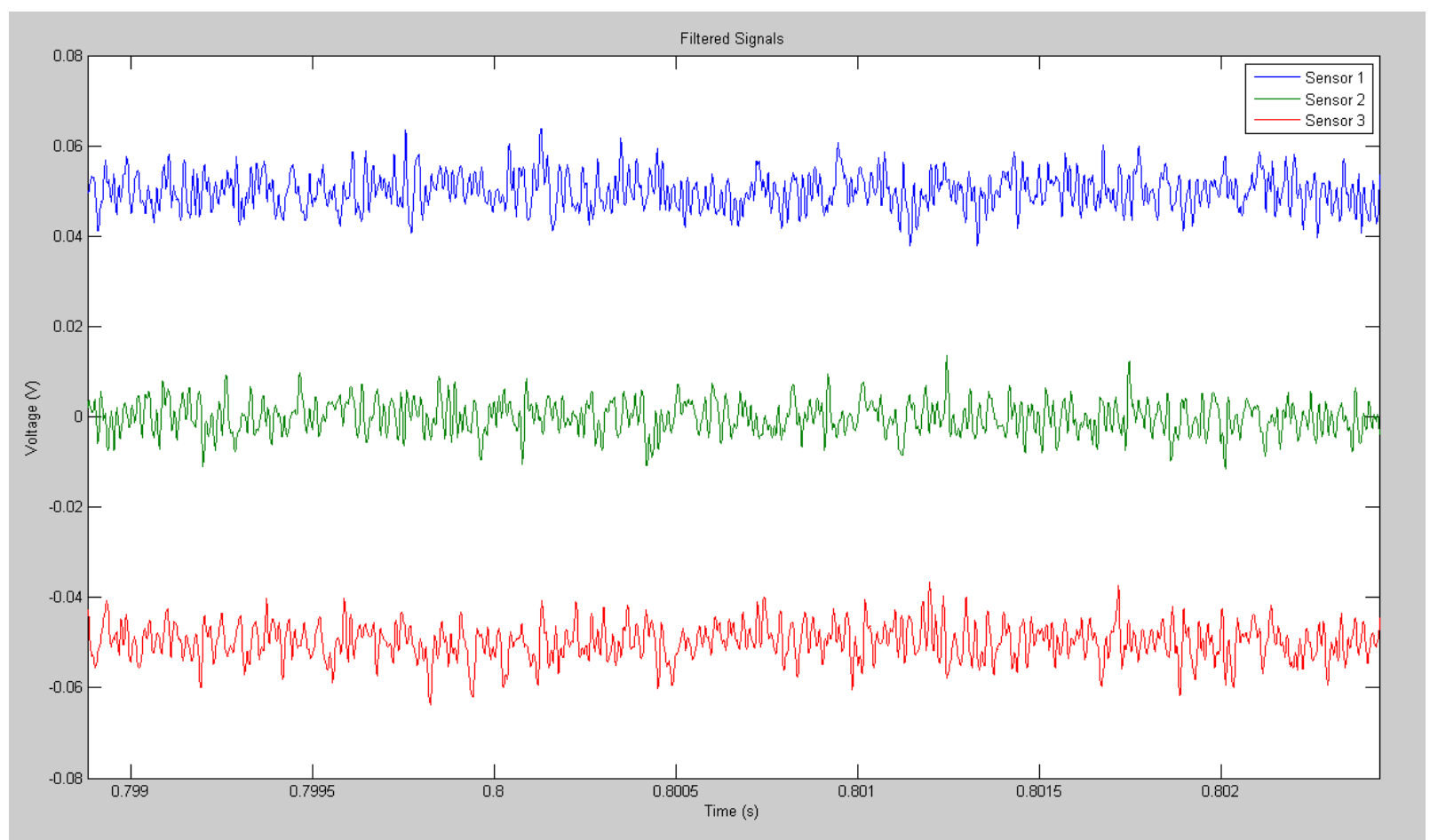

Figure 16: Array \#1 (top): Post processed signals - zoomed in view 
Intelligent Fiber Optic Sy stems Corp. (IFOS) DOE STTR GRANT No.: DE-

SC0001020

Interim Report \#4

\subsubsection{Array \#2 (Bottom)}

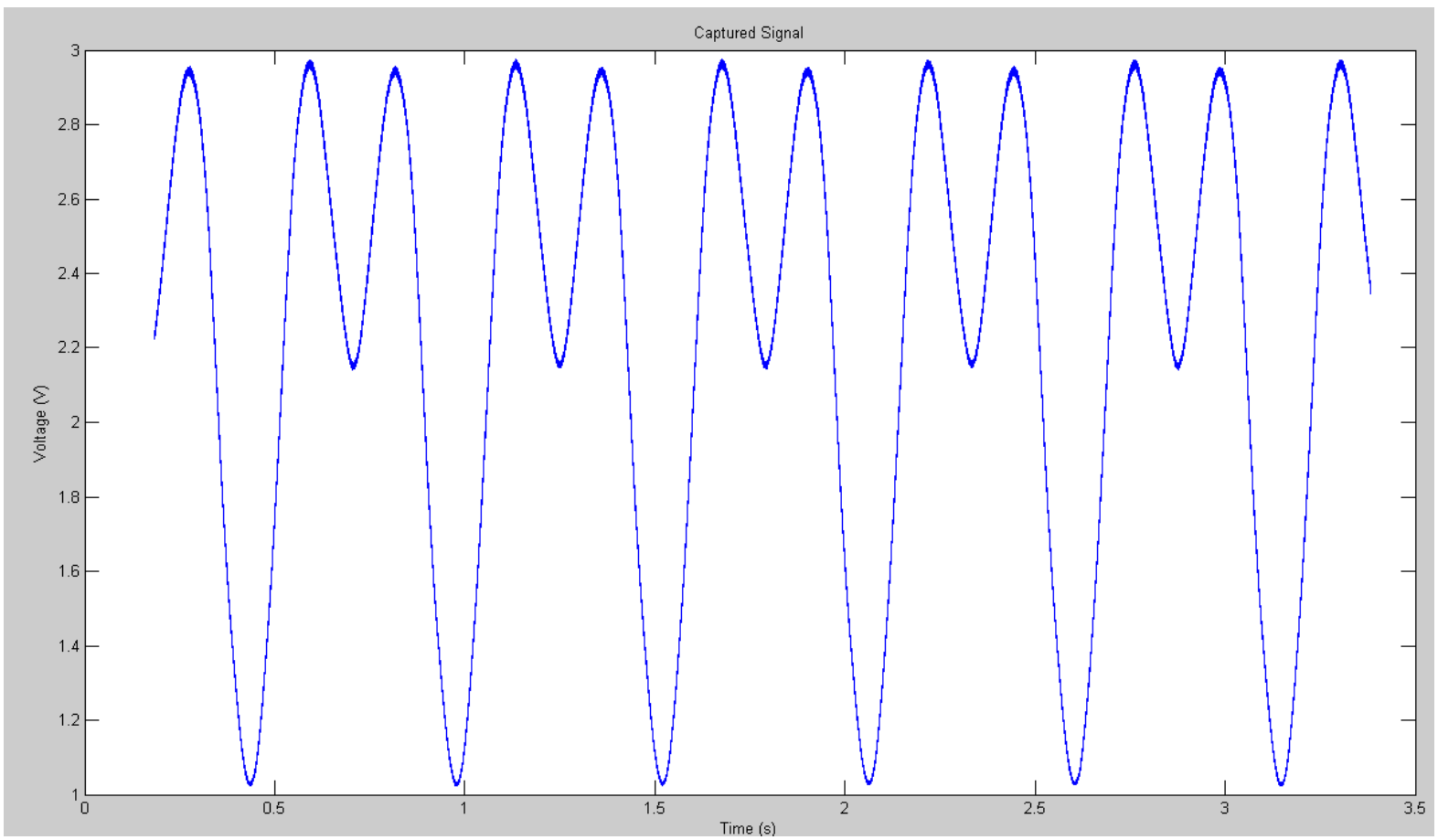

Figure 17: Array \#2 (Bottom): Original signal from FBG \#1 (Ch. 9)

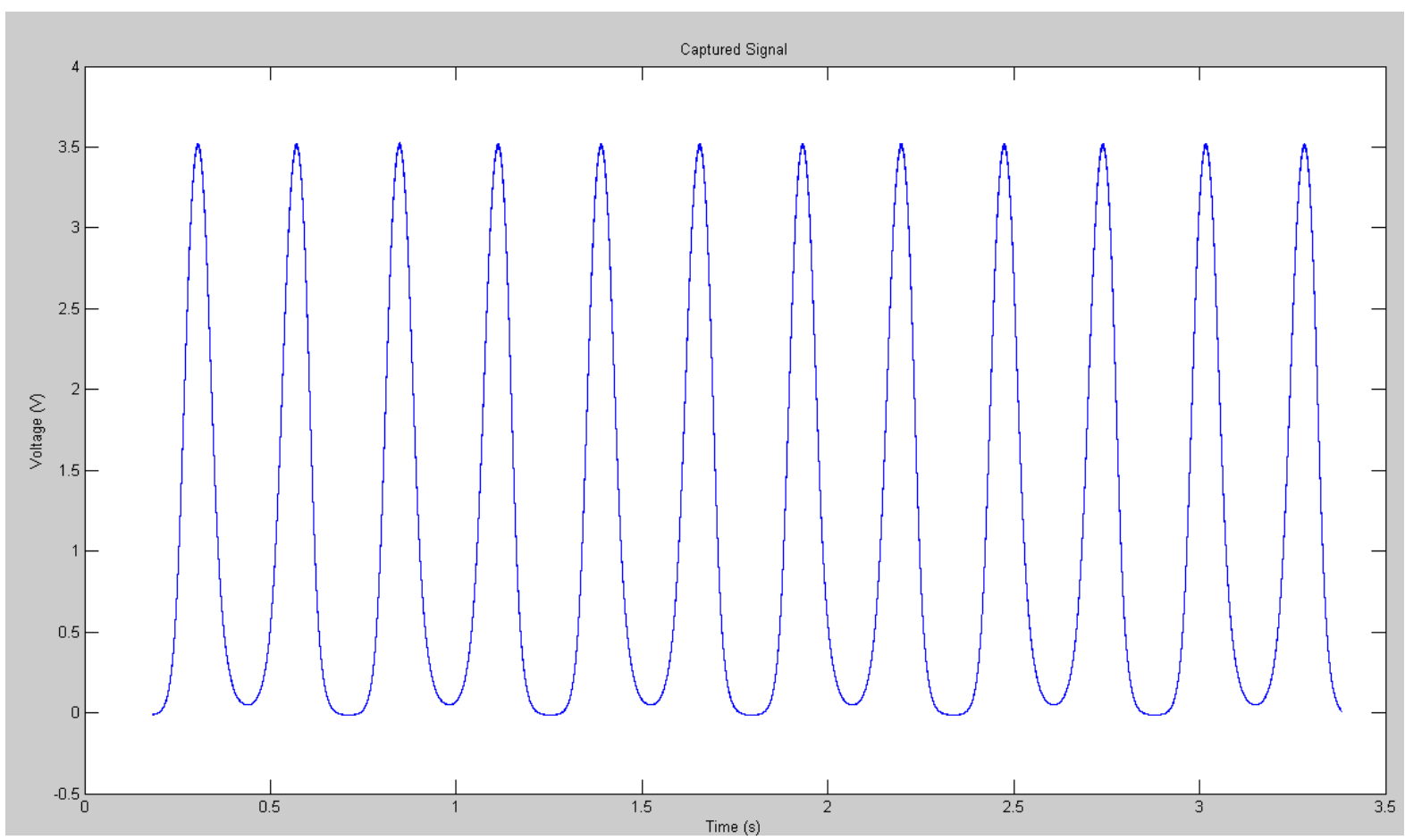

Figure 18: $\quad$ Array \#2 (Bottom): Original signal from FBG \#2 (Ch. 3) 
Intelligent Fiber Optic Sy stems Corp. (IFOS) DOE STTR GRANT No.: DE- 
Intelligent Fiber Optic Sy stems Corp. (IFOS) DOE STTR GRANT No.: DE-

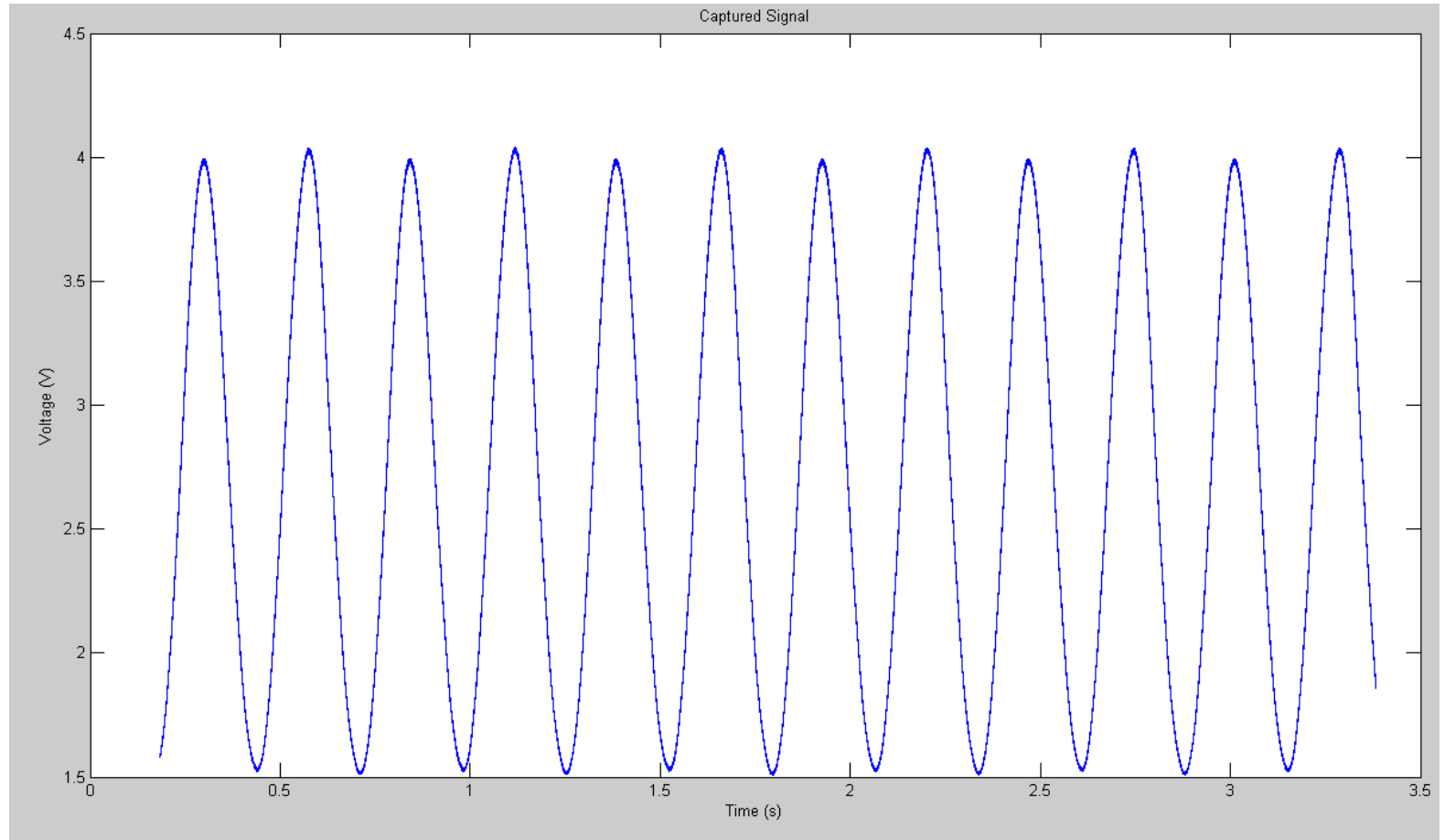

Figure 19: $\quad$ Array \#2 (Bottom): Original signal from FBG \#3 (Ch. 14)

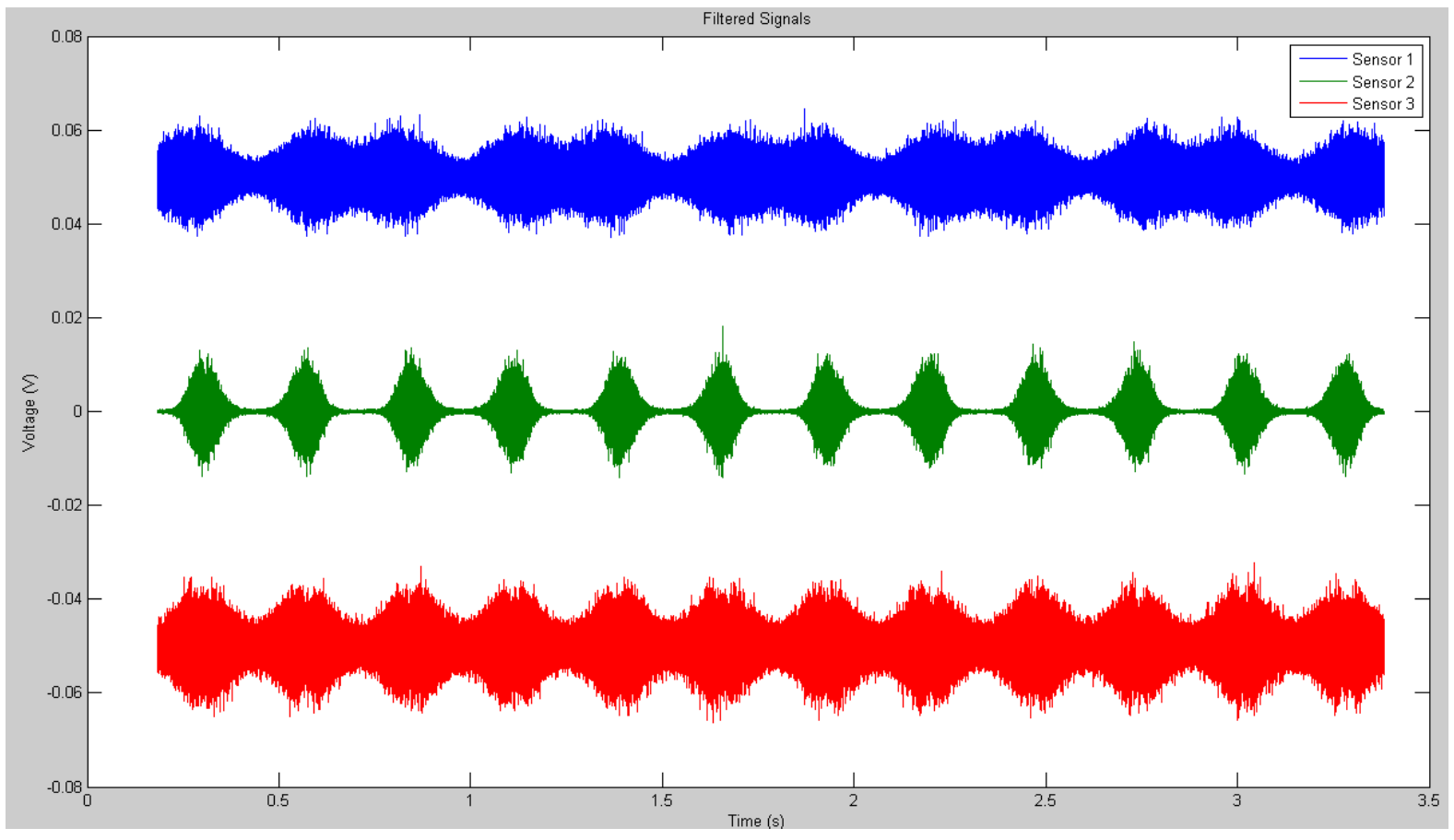

Figure 20: Array \#2 (Bottom): Post processed signals (Ch 9 plot is offset by $0.05 \mathrm{~V}$ and $\mathrm{Ch} 14$ plot is offset by $-0.05 \mathrm{~V}$ for a clearer view) 
Intelligent Fiber Optic Systems Corp. (IFOS) DOE STTR GRANT No.: DE-

$-:$

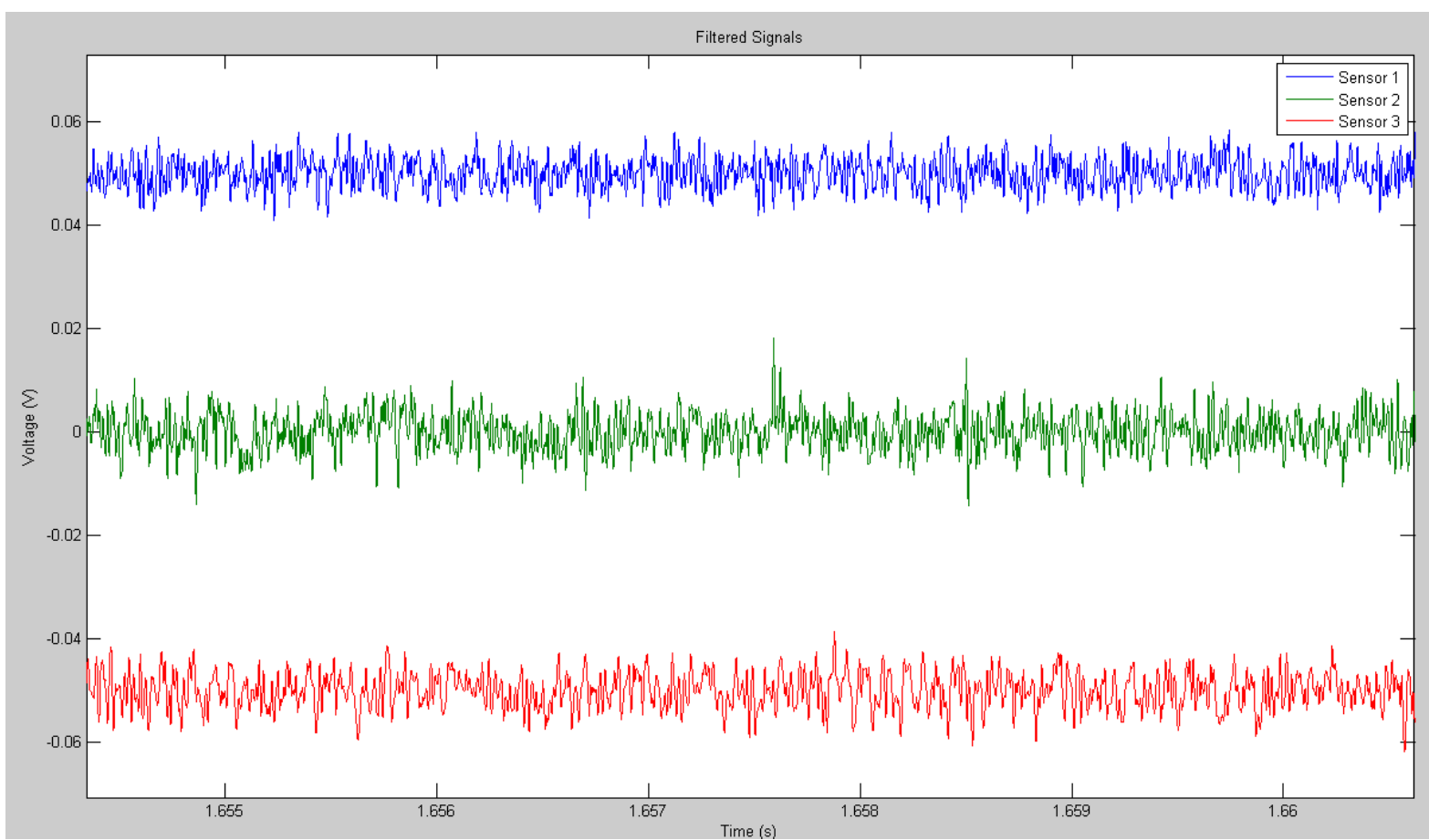

Figure 21: Array \#2 (Bottom): Post processed signals - zoomed in view

\subsubsection{Conclusion}

The results in terms of $\mathrm{AE}$ events after the data post processing are inconclusive and require further and more advanced post processing methods. Currently, we are in the process of exploring one of the more advanced data processing methods. 
Intelligent Fiber Optic Sy stems Corp. (IFOS) DOE STTR GRANT NO.: DE-

\section{AU Final Report for IFOS Project}

\section{Summary}

The experimental and modeling programs implemented by Auburn University during Phase I of this project are detailed in this report. Strain measurements monitored and recorded by Fiber Bragg Grating (FBG) sensors, which were mounted on composite coupons, during standard static and dynamic loading tests agreed with those obtained through other standard measuring devices. These experimental results were also confirmed through results of finite element models of the tested composite coupons. Effects of existing defects in the coupons on some fundamental structural properties, such as natural frequencies, were investigated. Also, a finite element model for a wind turbine blade was developed utilizing results of the experimental program for the purpose of suggesting optimum locations for the FBG sensors to be mounted or imbedded in the blade.

Results of both experimental and modeling work performed by AU provide confidence in the utilization of FBG sensors for remote monitoring of turbine wind blades and confirm the suitability of these sensors in tracing the behavior of the blades at different operating conditions and in detecting any defects or cracks within the blade structure.

\section{Objectives}

The first objective of the work conducted at AU in this project was to set and run an experimental program to validate the operation and accuracy of the FBG sensors mounted on composite coupons under standard cases of static and dynamic loadings. Static tests included tensile and four-point bending tests to determine elastic properties of the composite coupons and monitor their elastic and flexural responses to the applied loads. Dynamic tests included impact and vibration tests to evaluate the mode shapes and natural frequencies of the composite coupons.

The second objective was aimed at identifying the location and extent of the defects or damage in the blade structure through a model based approach. A baseline model will be developed and validated with experimental results from vibration testing. This includes correlating both natural frequencies and modal vibration amplitudes. Vibration data from intentionally damaged samples will be collected and compared to predicted results from the baseline model in order to assess sensitivity to the magnitude and location of defects.

The third objective was to develop finite element model of a wind turbine blade and examine the model under standard cases of static and dynamic loading for the purpose of locating optimum placement locations for the FBG sensors based on maps of the stress-strain distributions for the model. Main material properties utilized in this model (e.g., Young's modulus, Poison's ratio, and damping factor) were first verified through a group of finite element runs on models of the composite coupons similar to those used in the experimental program. 
Intelligent Fiber Optic Sy stems Corp. (IFOS) DOE STTR GRANT No.: DE-

\section{SC0001020}

Interim Report \#4

\section{Experimental Program \\ 3.1 Materials and Manufacturing}

The composite material chosen for the experimental program was T-30o carbon fibers and epoxy matrix, composed of EPON 828 resin and EPICURE 3140 hardener with the ratio of 100:75 by weight. These are typical materials commonly used by the composite industry. During manufacturing process, six layers of fabric, in the form of a broken twill, were coated with matrix and manually placed in a mold. The mold was compressed with 0.5 ton pressure and heated at temperature of $120^{\circ} \mathrm{C}$ for 2 hours using a Hydraulic compressor, Genesis Series 15, G30 H 15B, Wabash MPI. Post curing was conducted by placing the composite in an oven at $60^{\circ} \mathrm{C}$ for 24 hours. Two flat square plates with nominal dimensions of $250 \times 250 \times 3 \mathrm{~mm}$ were manufactured. Plate 1 was manufactured as a typical composite while plate 2 was manufactured such that half of it contained planned defects of delaminations and holes while the other half was free of defects. The plates were cut into coupons and edges were polished to remove the effect of cutting and ensure that no stress concentration would impact the results of the tests. One FBG sensor was mounted (by IFOS) on the surface of each coupon at its mid-length, as shown in Figure AU-1.

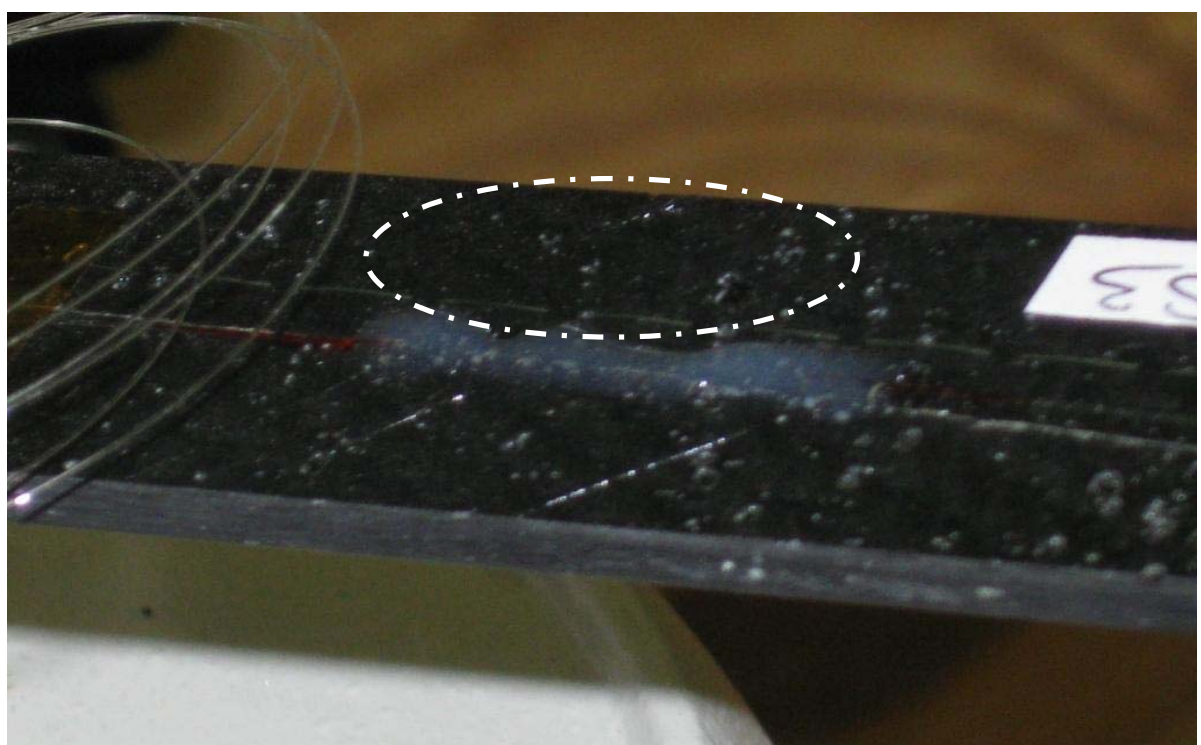

\subsection{Test Plan}

The testing program consisted of two sets of testing a) static tests which includes a tensile test and a four-point bending test to monitor the stress-strain and flexural responses of the composite coupons, and b) dynamic tests which include impact and vibration tests to study the mode shapes and natural frequencies of the composite coupons. Dimensions of the coupons are listed in Table AU-1. 
Intelligent Fiber Optic Sy stems Corp. (IFOS) DOE STTR GRANT No.: DE-

SC0001020

Interim Report \#4

Table AU-1: Dimensions of composite coupons of Plate 1

\begin{tabular}{|l|c|c|c|c|c|c|c|c|c|}
\hline Test & \multicolumn{3}{|c}{ Tensile } & \multicolumn{3}{c|}{$\begin{array}{c}\text { Four-point } \\
\text { bending }\end{array}$} & \multicolumn{3}{c|}{ Dynamic } \\
\hline $\begin{array}{l}\text { Coupon } \\
\text { ID }\end{array}$ & $1 \mathrm{~T} 1$ & $1 \mathrm{~T} 2$ & $1 \mathrm{~T} 3$ & $1 \mathrm{~F} 1$ & $1 \mathrm{~F} 2$ & $1 \mathrm{~F} 3$ & $1 \mathrm{D} 1$ & $1 \mathrm{D} 2$ & $1 \mathrm{D} 3$ \\
\hline $\begin{array}{l}\text { Length } \\
\text { (mm) }\end{array}$ & 128.78 & 128.78 & 128.78 & 91.64 & 91.64 & 91.64 & 220 & 220 & 220 \\
\hline $\begin{array}{l}\text { Width } \\
\text { (mm) }\end{array}$ & 12.08 & 12.27 & 12.78 & 12.07 & 13.03 & 12.32 & 25.25 & 25.12 & 24.33 \\
\hline $\begin{array}{l}\text { Thick } \\
\text { (mm) }\end{array}$ & 3.07 & 3.29 & 3.3 & 3.33 & 3.02 & 3.02 & 3.21 & 3.18 & 3.23 \\
\hline
\end{tabular}

\subsubsection{Tensile Tests}

Tests were conducted on "Instron 5565", as shown in Figure AU-2. Each coupon was subjected to three sets of linearly increasing tensile loads of maximum values 1000 , 2000, and 3000 N. Deformation was simultaneously monitored and recorded from the FBG sensor through the "I-sense 48000 Multi Array interrogator" unit with the "Mini I-sense" software (supplied by IFOS) and the Instron video extensometer (at AU labs). During the operation of the video extensometer, a digital camera and a real-time image processing software measure the distance between two dots marked on the coupon and track the change in this distance as the coupon extends. The initial distance between the two dots is considered as the gauge length while the change is considered the extension. At the same time the load cell measures the instantaneous load. Both load and real extension from the video extensometer are used to construct the stress-strain diagrams. Three coupons 1T1, 1T2, and 1T3 were tested. Results are presented in Section 4.1.1. 
Intelligent Fiber Optic Sy stems Corp. (IFOS) DOE STTR GRANT No.: DE-

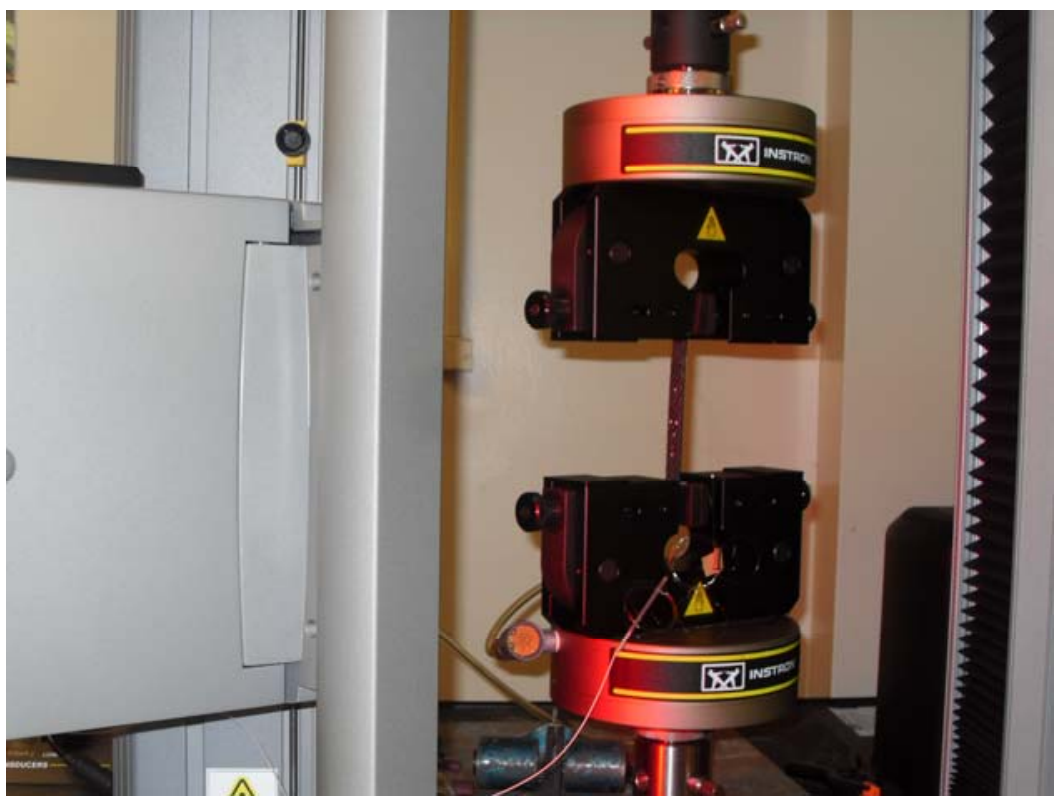

Figure AU-2: Tensile test of a composite coupon marked with two dots

\subsubsection{Four-point bending Tests}

Tests were conducted on an "Instron 5565" equipped with a flexure test fixture, as shown in Figure AU-3. Each coupon was subjected to three sets of linearly increasing flexural loads up to 50,100, and $150 \mathrm{~N}$. The span between supports was taken to be $530 \mathrm{~mm}$ with load-to-support span ratio of 0.5, as shown in Figure AU-4. Deformation of each coupon was simultaneously monitored through the interrogator unit and the Instron output. The three coupons $1 \mathrm{~F} 1,1 \mathrm{~F} 2$, and $1 \mathrm{~F}_{3}$ were tested. Results are presented in Section 4.1.2. 
Intelligent Fiber Optic Sy stems Corp. (IFOS) DOE STTR GRANT No.: DESC0001020

Interim Report \#4

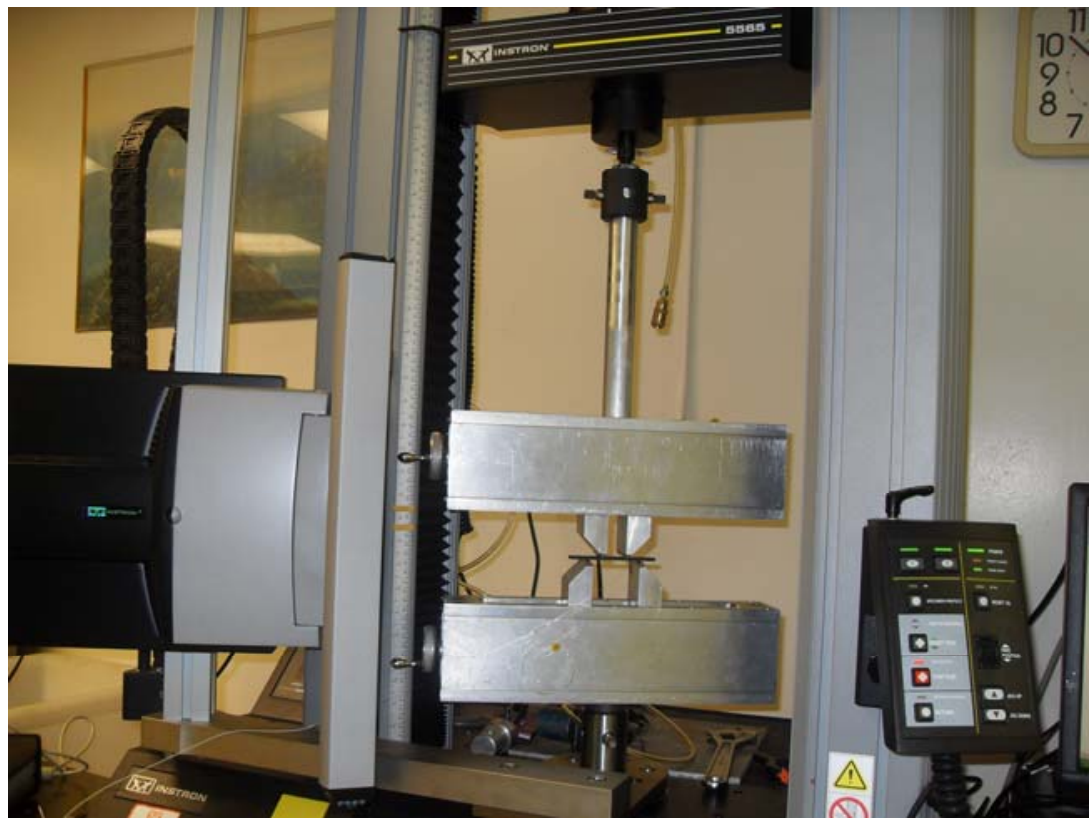

Figure AU-3: Four-point bending of a composite coupon.

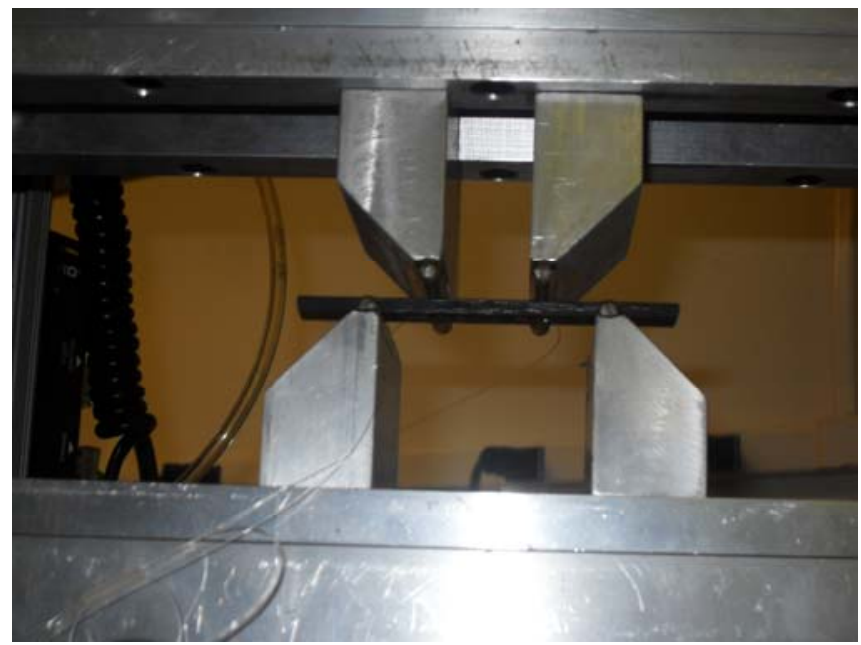

Figure AU-4: Load-to-support span ratio of 0.50 in four-point bending tests.

\subsubsection{Dynamic tests}

Tests were conducted using the following equipment:

- Shaker:

LDS V408

- Amplifier:

LDS PA500L

- Sensor head:

Polytec OFV 353

- Controller:

Polytec OFV 2610

- Analyzer:

HP 35665A 
Intelligent Fiber Optic Sy stems Corp. (IFOS) DOE STTR GRANT No.: DE-

SC0001020

Interim Report \#4

Figure AU-5 is a schematic of the equipment set up. The clamp which is used for holding the coupon is attached to the moving platform of the shaker. The shaker vibrates vertically based on the input from the amplifier, which amplifies the source signal generated by the analyzer. Apart from generating source signal (the type, amplitude and frequency range of which can be specified), the analyzer receives measured data from the vibrometer controllers, processes them and display desired results. The input and output vibrometer signals are fed to channels 1 and 2 of the analyzer, respectively. Two laser vibrometers are used, one for the input signal, that is the rate at which the shaker moving platform and the clamp vibrates, and one for the output signal, that is the rate at which the test sample vibrates. Each vibrometer consist of a sensor head and a controller. The controller provides power to the sensor head and decodes the measured signal from the sensor head. Pictures of the above set up are shown in figures AU-6 to AU-9.

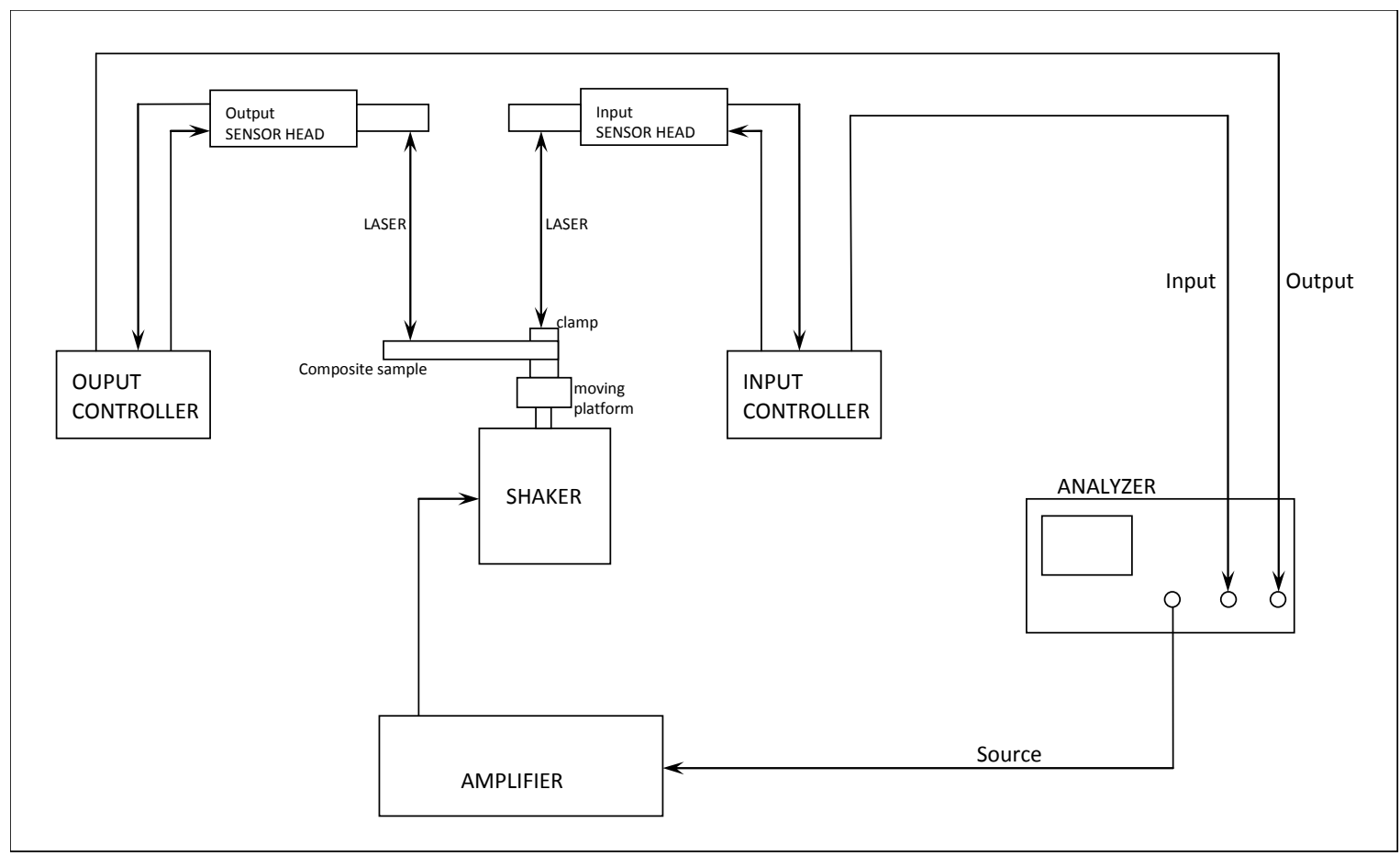

Figure AU-5: Schematic of equipment set up. 
Intelligent Fiber Optic Sy stems Corp. (IFOS) DOE STTR GRANT No.: DESC0001020

Interim Report \#4

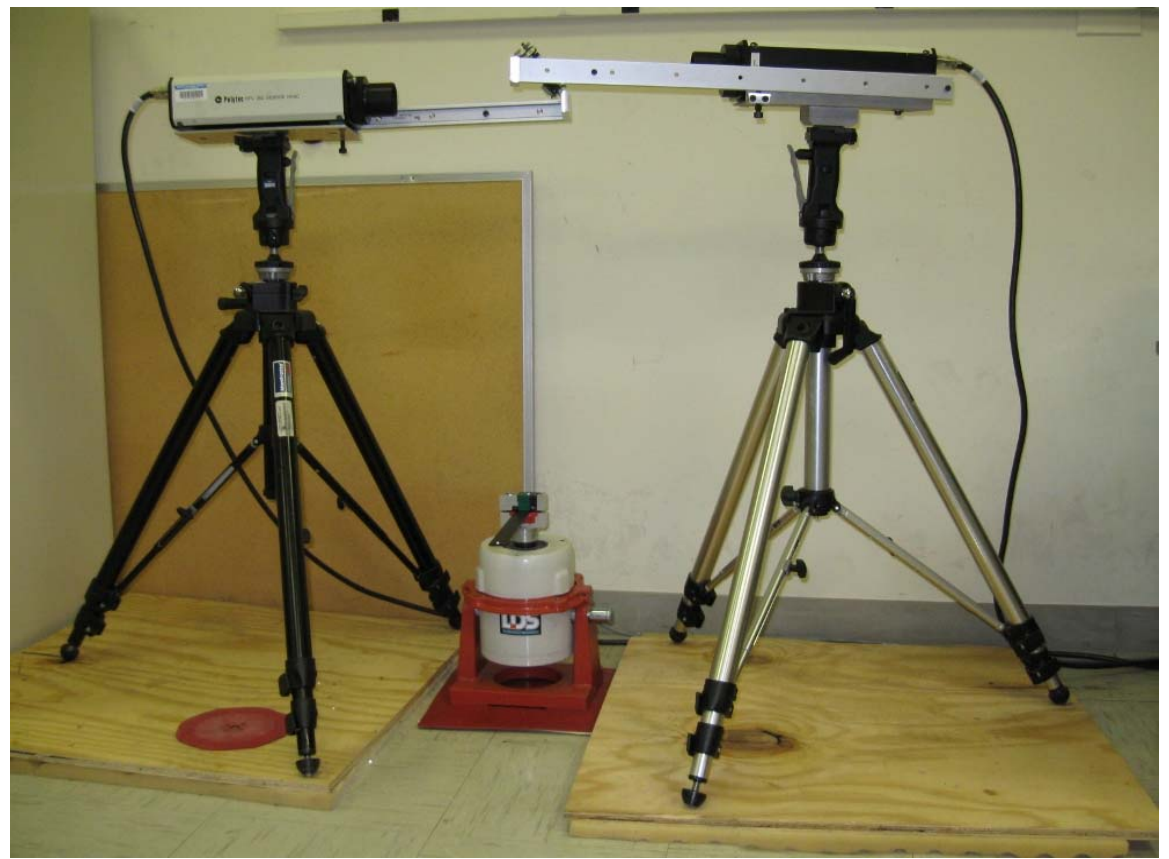

Figure AU-6: Two laser sensor heads with the shaker in between.

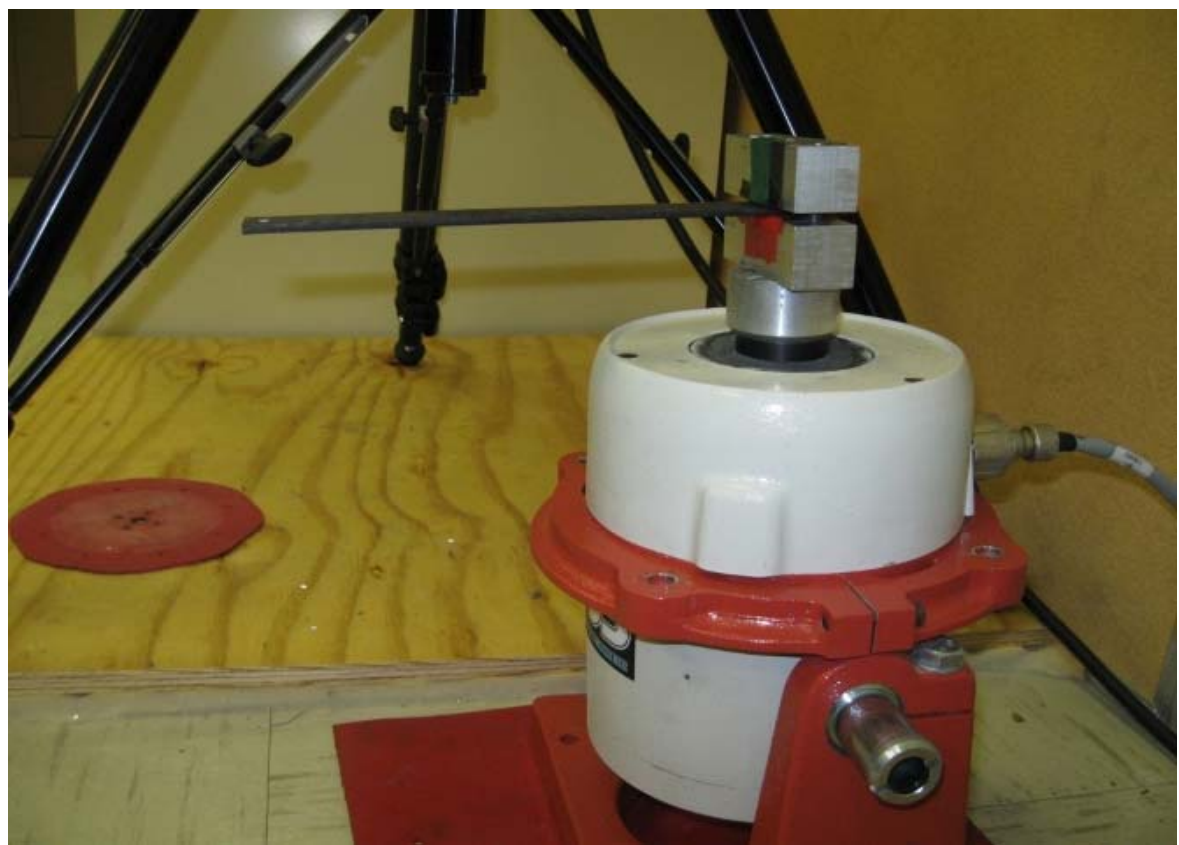

Figure AU-7: A test piece in between clamp, which is attached to moving platform of the shaker. 
Intelligent Fiber Optic Sy stems Corp. (IFOS) DOE STTR GRANT No.: DE-

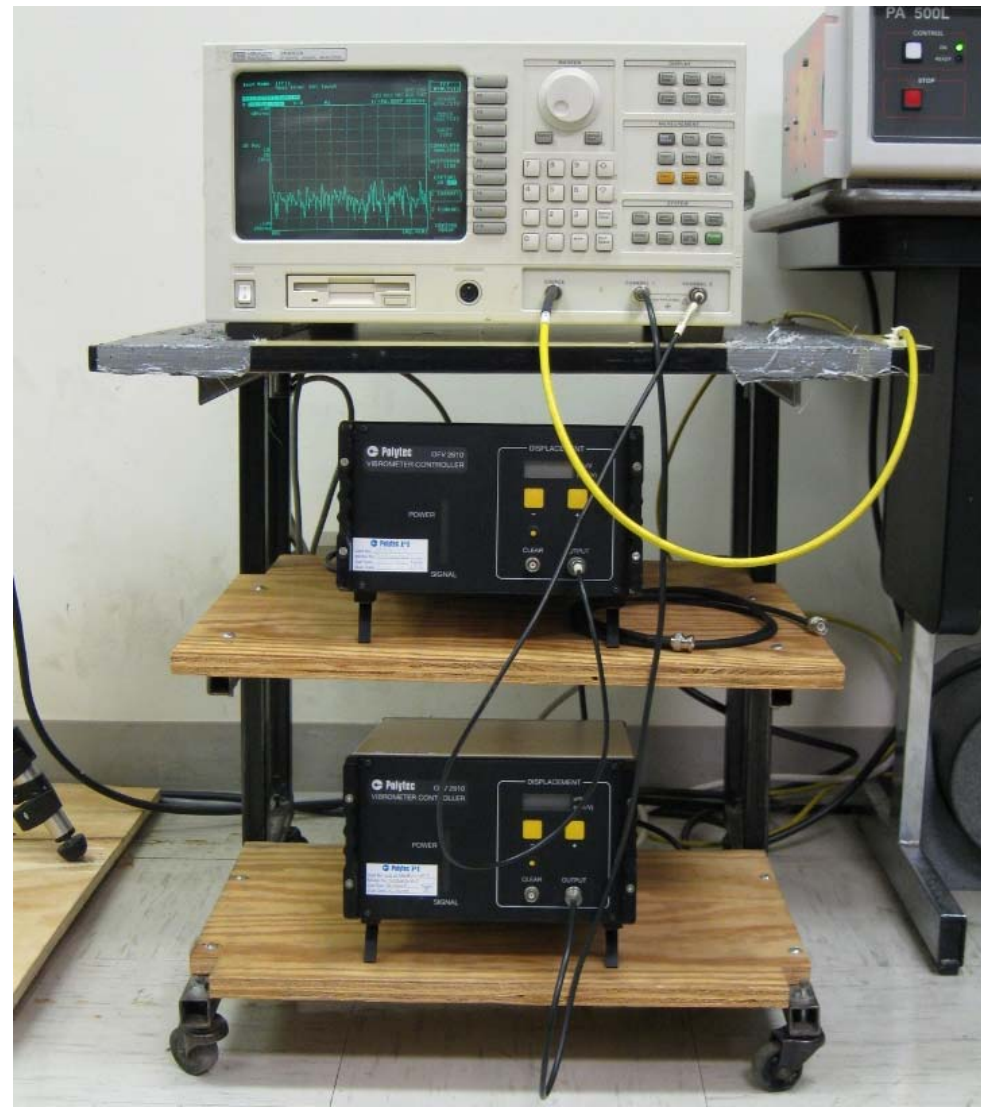

Figure AU-8: The analyzer connected to the vibrometer controllers and the yellow cable for the source signal.

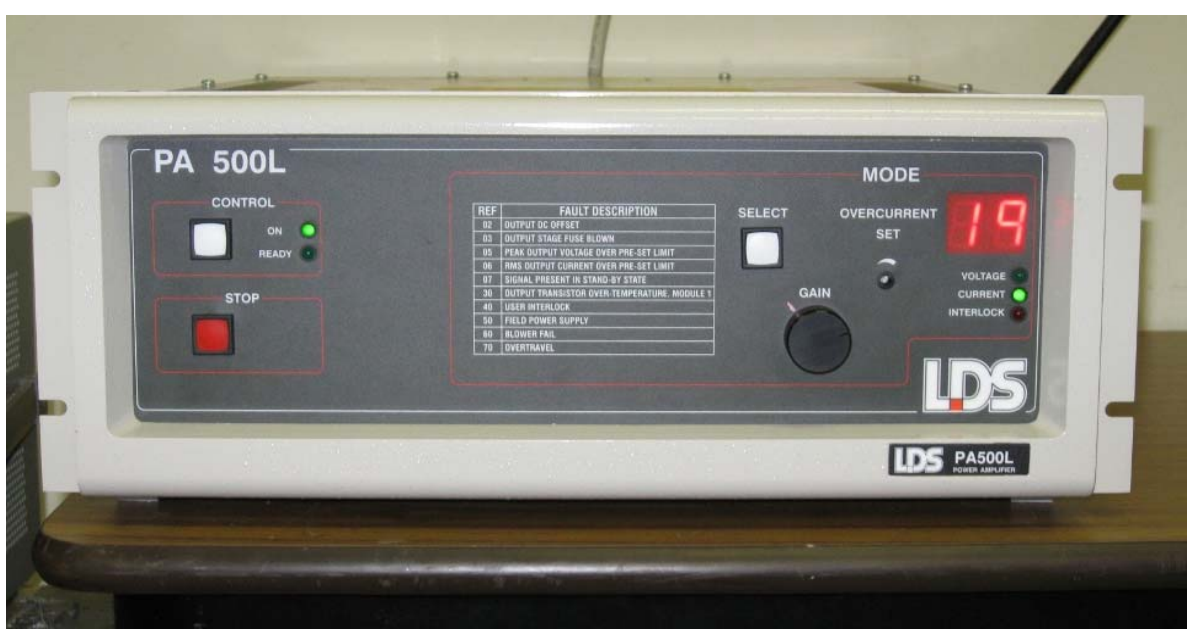

Figure AU-9: The amplifier.

The sensor head emits a laser beam which is reflected from the surface being measured. For this purpose a reflective sticker was placed on the clamp and the composite coupon. The coupon was clamped to the moving platform and the laser beams were focused at the center on their respective sticker so as to minimize noise in 
Intelligent Fiber Optic Sy stems Corp. (IFOS) DOE STTR GRANT NO.: DE-

SC0001020

Interim Report \#4

the signal. The controller setting was set at a displacement of $320 \mathrm{um} / \mathrm{volt}$. On the analyzer, a pink noise which consists of all the frequencies in the selected range acting simultaneously was selected as a source type with an amplitude of $50 \mathrm{mV}$ RMS and a frequency range of $0-3200 \mathrm{~Hz}$. This signal was amplified and fed to the shaker and the transfer function of the output/input was displayed on the analyzer. To reduce the effect of noise, 20 averages of the transfer function were conducted.

Three different tests were performed on coupons 1D1 and 1D3 as follows:

- $\quad$ Impact test: Where a small spanner was used to give an impact to the clamp and the transient response recorded.

- Vibration test: where the sample was made to vibrate at the above mentioned source signal and the vibration response recorded.

- $\quad$ End Displacement test: the free end was displaced vertically, and the transient response of the coupon was recorded. Then using only the output vibrometer connected to channel 1 of the analyzer, time trace was recorded for 2 seconds to allow the displacement to end.

Results of the tests are presented in Section 4.1.3.

\subsection{Finite element modeling}

Experimental results of the static and dynamic tests were examined numerically with a finite element analysis. Models were built, using ANSYS, for tensile, four-point bending, and dynamic test coupons. pcGINA ${ }^{\odot}$ software was used to determine the elastic properties of the composite. The orthotropic material properties, taken from experimental data as well as pcGINA runs, were input in ANSYS and "SOLID95" elements were used to create the mesh of the model. Results of ANSYS runs for the coupon models are documented in Section 4.1.

The NuMAD software (from Sandia National Labs) was used to build a wind turbine blade model. Geometrical aspects of the blade model were taken from literature while the composite elastic properties were taken similar to those of the coupon models. The model was incorporated into ANSYS and meshed using the shell element "SHELL99". Several FEA runs were performed on the model under different cases of static flexural, lateral, and torsional loadings. Results of these runs are presented in Section 4.1.4.

\section{Results and Discussion}

4.1 Results

\subsubsection{Results of tensile tests and FEA model}

Tensile tests were performed with the FBG sensors mounted on two coupons from plate 1: 1T1 and 1T2. Tensile loads on the Instron were increased up to 1000, 2000, and $3000 \mathrm{~N}$ in three sets of tests. Samples of the strain results recorded on both the I-sense interrogator and Instron video extensometer for coupon 1T2 are shown in Figures AU10 and AU-11, respectively. 
Intelligent Fiber Optic Sy stems Corp. (IFOS) DOE STTR GRANT No.: DE-

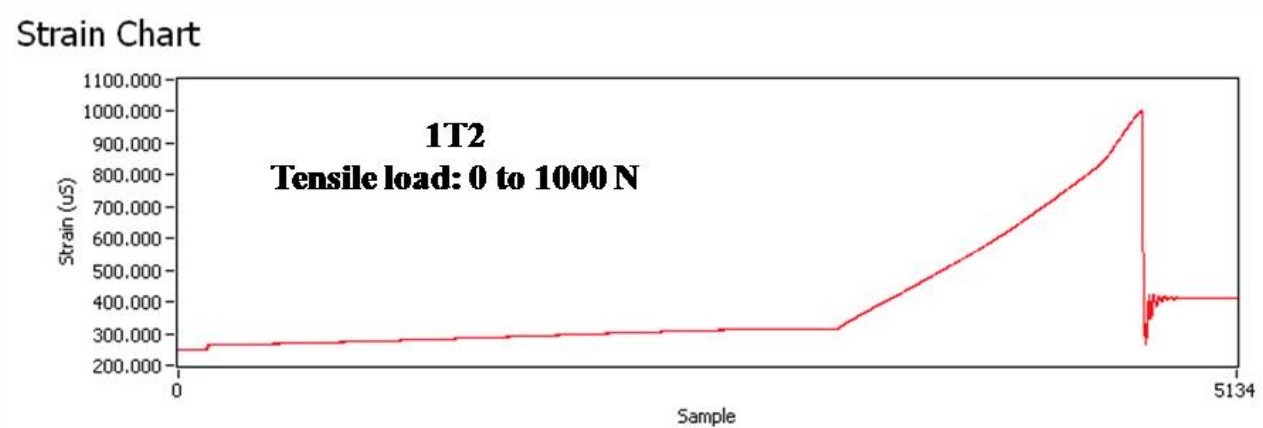

Strain Chart

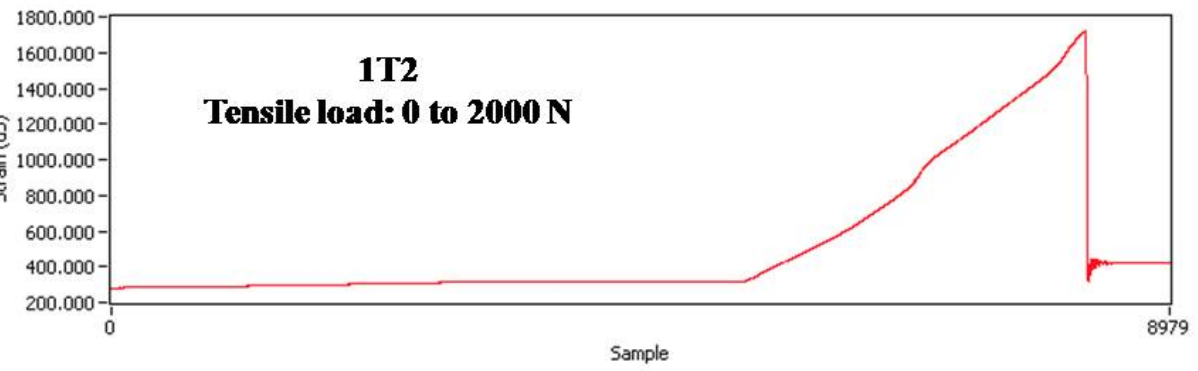

Strain Chart

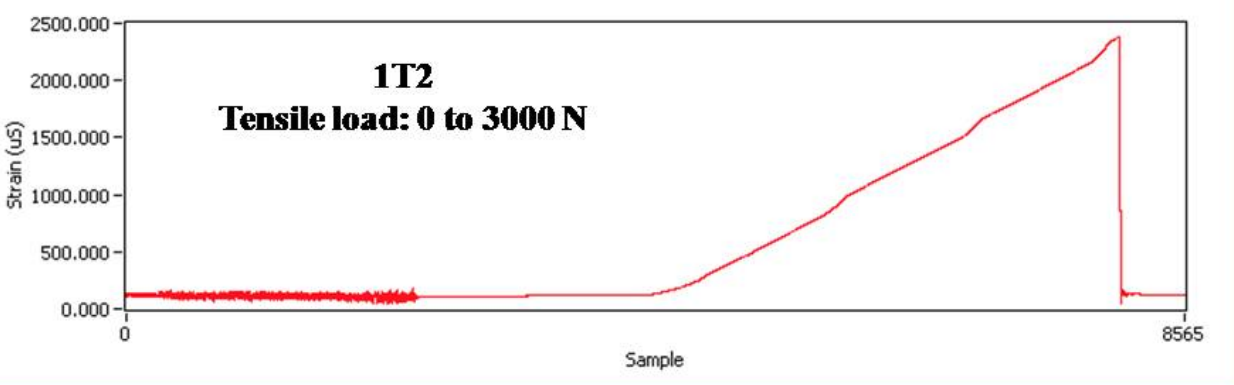

Figure AU-10: Strain results recorded by FBG sensors on the I-sense interrogator and plotted by the Mini I-sense software for the tensile tests performed on coupon 1T2. 
Intelligent Fiber Optic Sy stems Corp. (IFOS) DOE STTR GRANT No.: DE-

Interim Report \#4
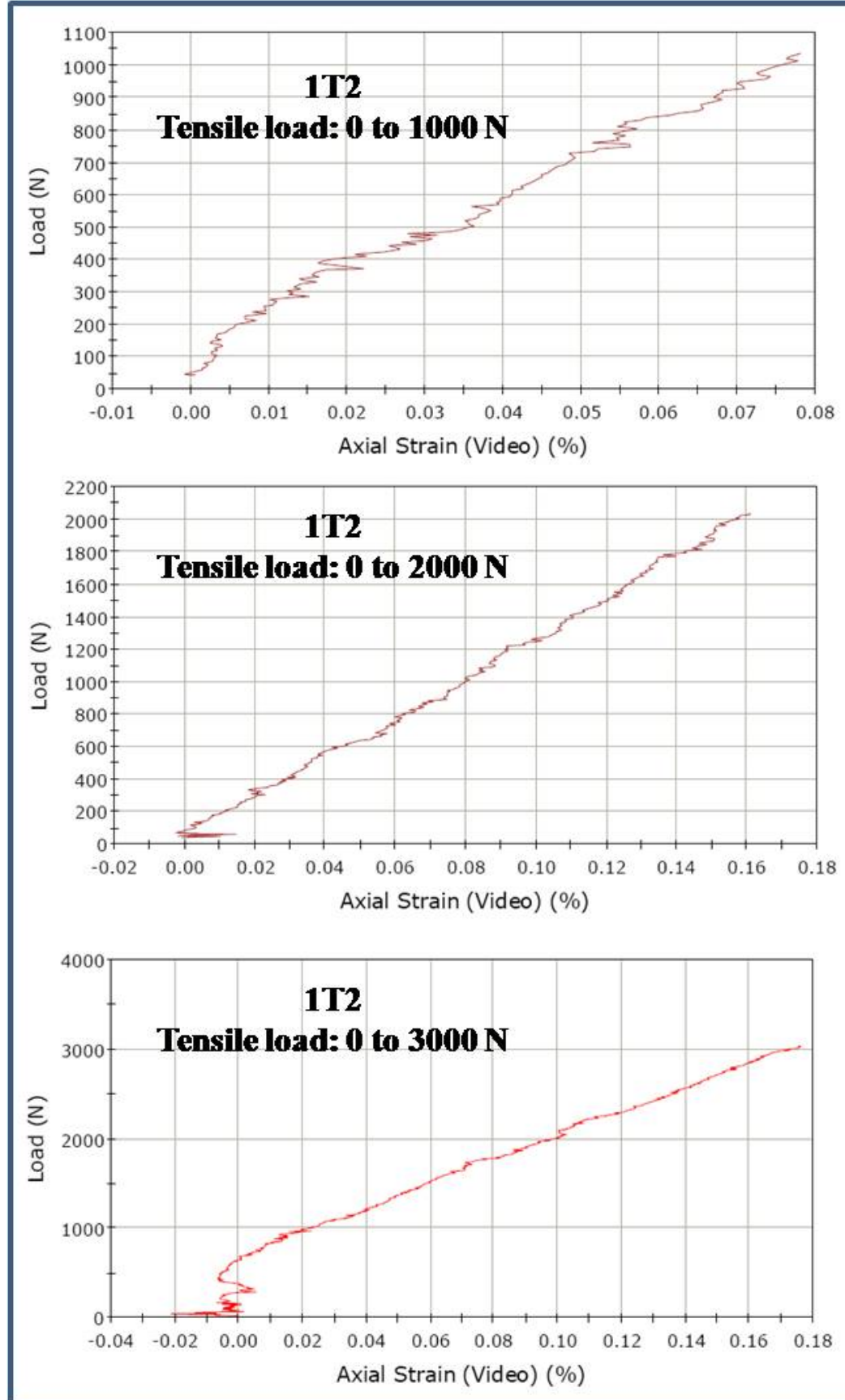

Figure AU-11: Strain results recorded on the Instron video extensometer for the tensile tests performed on coupon $1 T 2$.

A finite element model was built using ANSYS for coupon 1T3, as seen in Figure AU-12. The software pcGina $^{\circledR}$ was used to determine the elastic properties of the model based on a fiber volume fraction of $\mathbf{2 7 . 5 \%}$ which was calculated during the manufacture process of plate 1. These properties are listed in Table AU-2. 


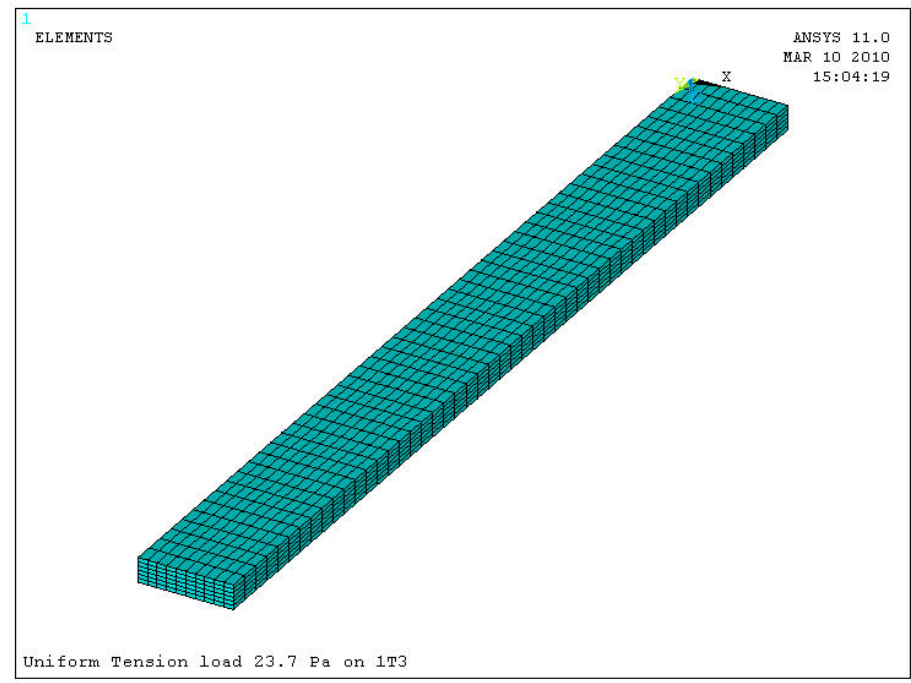

Figure AU-12: FEA model for coupon 1T3.

Table AU-2: Elastic properties of the FEA model for coupons of plate 1.

\begin{tabular}{|l|c|c|c|c|c|c|c|c|c|}
\hline Property & $\mathrm{E}_{\mathrm{x}}$ & $\mathrm{E}_{\mathrm{y}}$ & $\mathrm{E}_{\mathrm{z}}$ & $\mathrm{G}_{\mathrm{xy}}$ & $\mathrm{G}_{\mathrm{xz}}$ & $\mathrm{G}_{\mathrm{yz}}$ & $v_{\mathrm{xy}}$ & $v_{\mathrm{xz}}$ & $v_{\mathrm{yz}}$ \\
\hline Units & $\mathrm{G} \mathrm{Pa}$ & $\mathrm{G} \mathrm{Pa}$ & $\mathrm{G} \mathrm{Pa}$ & $\mathrm{G} \mathrm{Pa}$ & $\mathrm{G} \mathrm{Pa}$ & $\mathrm{G} \mathrm{Pa}$ & & & \\
\hline Value & 34.934 & 34.934 & 6.61 & 1.9867 & 1.916 & 1.916 & 0.0658 & 0.4815 & 0.4815 \\
\hline
\end{tabular}

The model was meshed into 3000 elements using the "SOLID 95" element type. The model was meshed into six layers of elements to accurately represent the six perform layers of the actual manufactured plate. The model was subjected to three different sets of tensile loads $(1000,2000$, and $3000 \mathrm{~N})$ with boundary conditions of a clamped-free bar.

Linearized stress-strain curves are plotted in Figure AU-13 to compare between strain measurements taken by both the Instron video extensometer and the FBG sensors together with the FEA model results. 
Intelligent Fiber Optic Sy stems Corp. (IFOS) DOE STTR GRANT No.: DE-

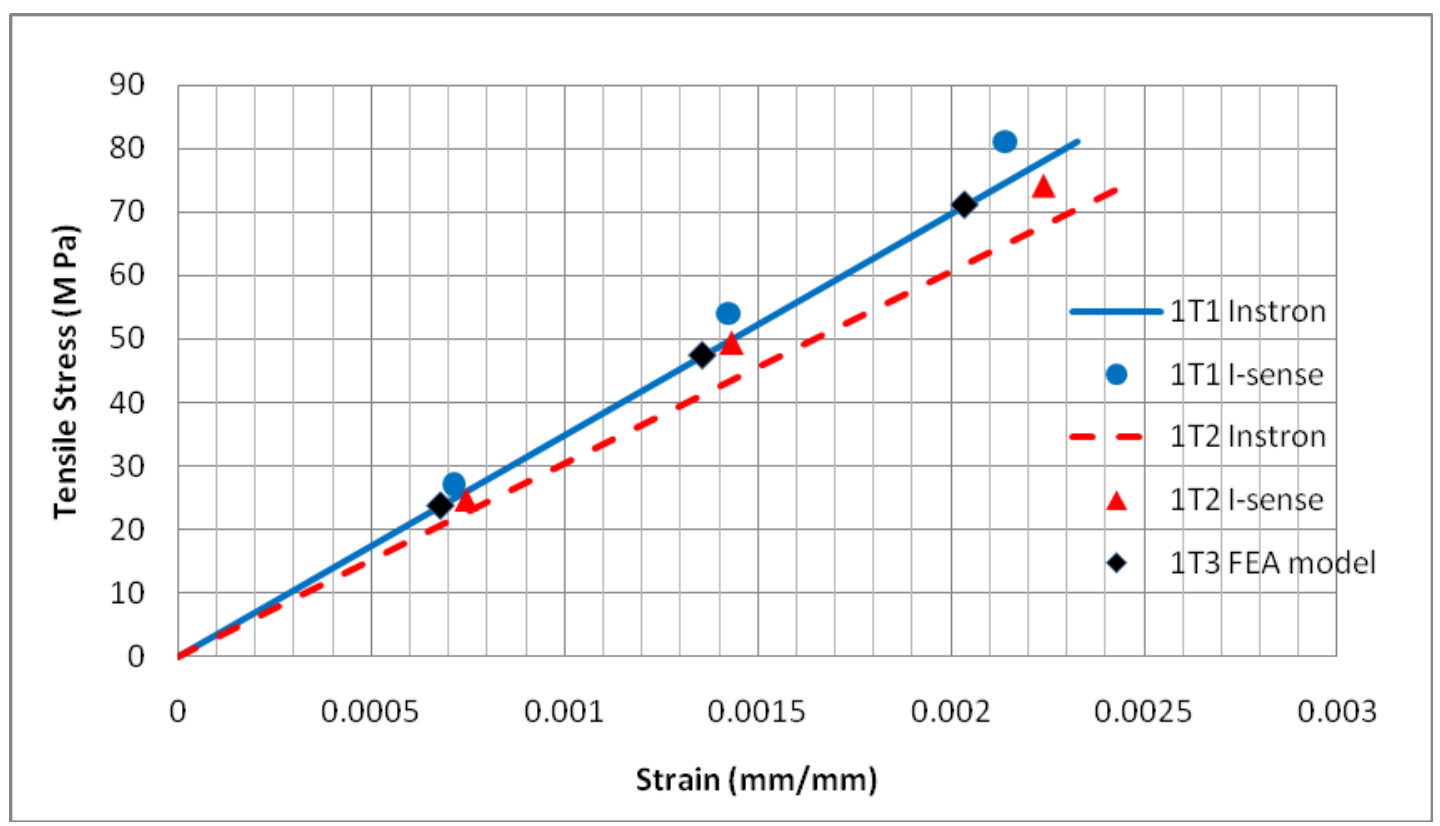

\section{Figure AU-13: Linearized stress-strain curves based on results of Instron video extensometer, FBG sensors, and FEA model in the tensile tests.}

As can be seen in the figure, all experimental and numerical results reasonably matched at such low strain values. This provides confidence in the accuracy of the FBG sensors and also in the FEA modeling procedures.

\subsubsection{Results of four-point bending tests and FEA model}

Four-point bending tests were performed with the FBG sensors mounted on three coupons from plate $1: 1 F_{1}, 1 F_{2}$, and $1 F_{3}$. The FBG sensor was placed on the other side of the two load noses at a location that is at the center between their points of application. Taking the Beam Theory as a reasonable approximation for such load scenario, the strain on the outer surface has a constant value anywhere between the two applied loads. If we take the support span to be $\mathrm{L}$ and the load span to be $\mathrm{X}$, as shown in Figure AU-14, then for the case of $\mathrm{X} / \mathrm{L}=0.5$ this value of constant strain, $\varepsilon_{0}$, would be given as:

$$
\varepsilon_{0}=\left(6 t / L^{2}\right) * d_{p}
$$

Where, $t$ is the thickness of coupon, $L$ is the support span, and $d_{p}$ is the vertical deflection of the coupon at the location of load P. It should be noted that $d_{p}$ is measured by the Instron output and may also be given by:

$$
\mathrm{d}_{\mathrm{p}}=\left(\mathrm{PL}^{3}\right) /(48 \mathrm{EI})
$$

Where, $\mathrm{P}$ is the applied load, $\mathrm{E}$ is Young's modulus, and I is the flexural inertia of the coupon. 
Intelligent Fiber Optic Sy stems Corp. (IFOS) DOE STTR GRANT No.: DE-

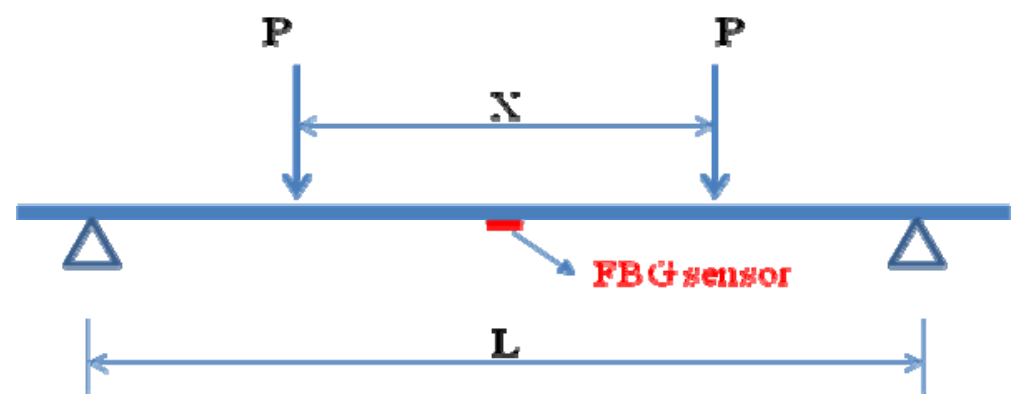

Figure AU-14: Schematic diagram for the four-point bending tests.

Flexural loads on the Instron were 50, 100, and $150 \mathrm{~N}$ in three sets of tests. Samples of the strain results recorded on both the I-sense interrogator and Instron output for coupon $1 \mathrm{~F} 2$ are shown in Figures AU-15 and AU-16, respectively.

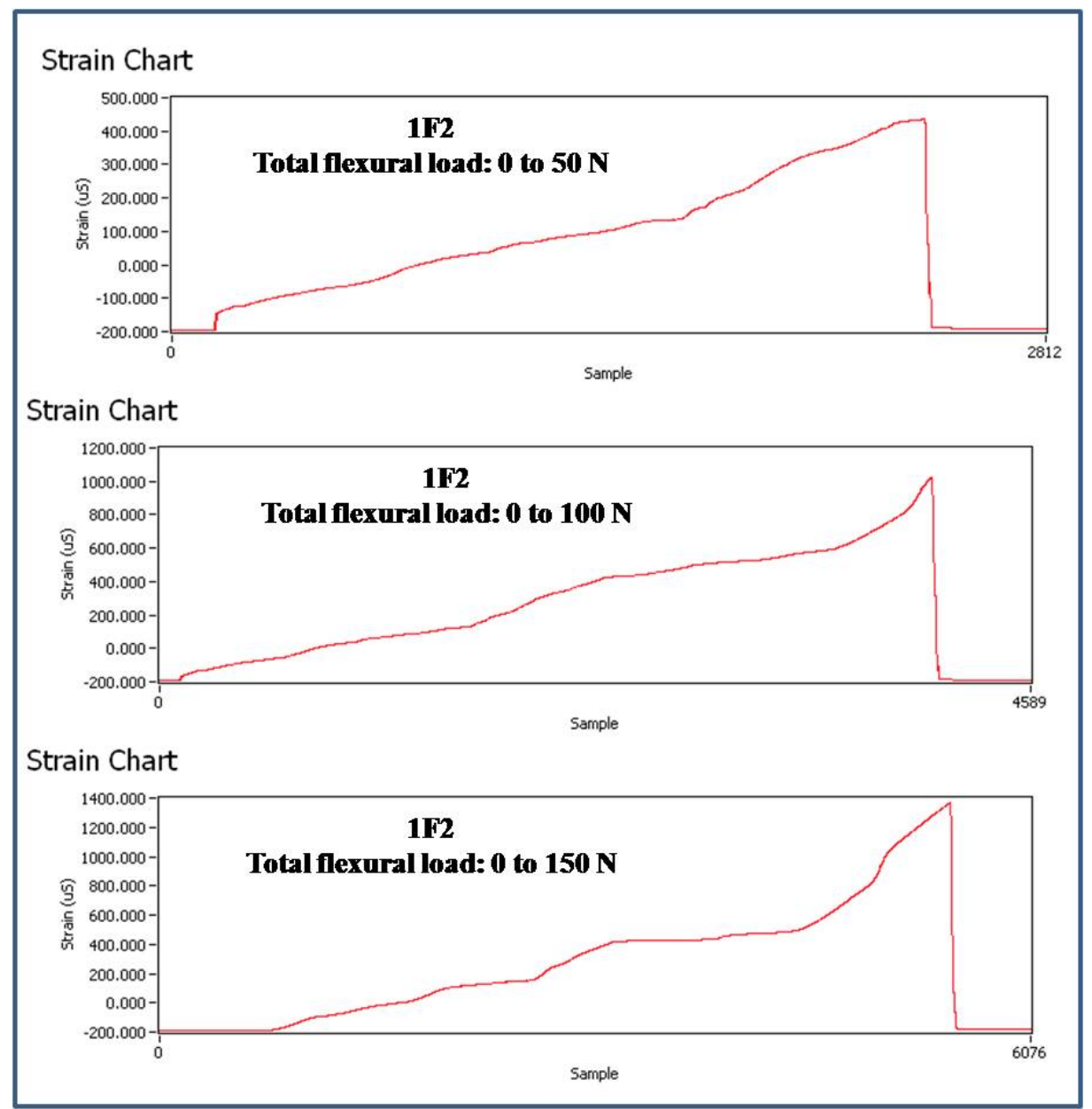

Figure AU-15: Strain results recorded by FBG sensors on the I-sense interrogator and plotted by the Mini I-sense software for the four-point bending tests performed on coupon $1 F 2$. 
Intelligent Fiber Optic Sy stems Corp. (IFOS) DOE STTR GRANT No.: DE-
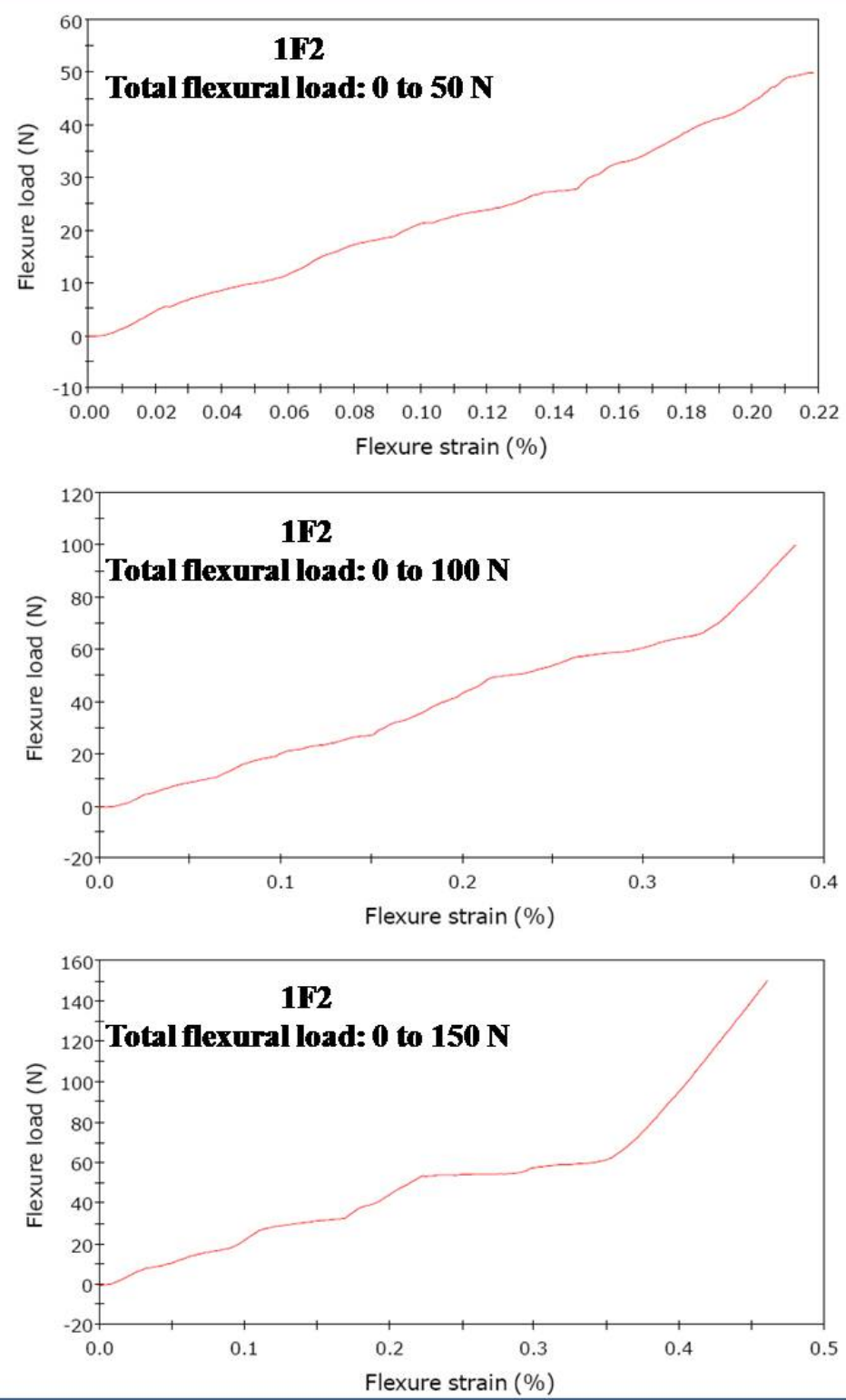

Figure AU-16: Strain results recorded on the Instron output for the fourpoint bending tests performed on coupon 1 F2.

A finite element model was built using ANSYS for coupon $1 \mathrm{~F}_{3}$, as seen in Figure AU-17. Elastic properties of the model were the same as in Table AU-2. The model was meshed into 3600 elements using the "SOLID 95" element type. The model was subjected to three different sets of flexural loads $(50,100$, and $150 \mathrm{~N})$ with boundary conditions of a simply supported bar, as shown in Figure AU-18. 
Intelligent Fiber Optic Sy stems Corp. (IFOS) DOE STTR GRANT No.: DE-

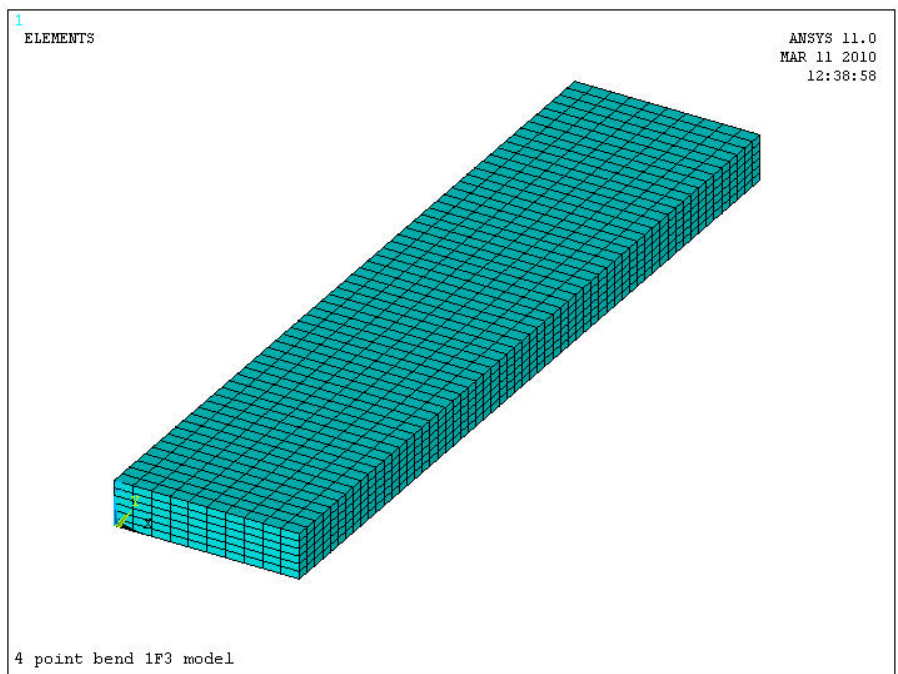

Figure AU-17: FEA model for coupon 1F3.

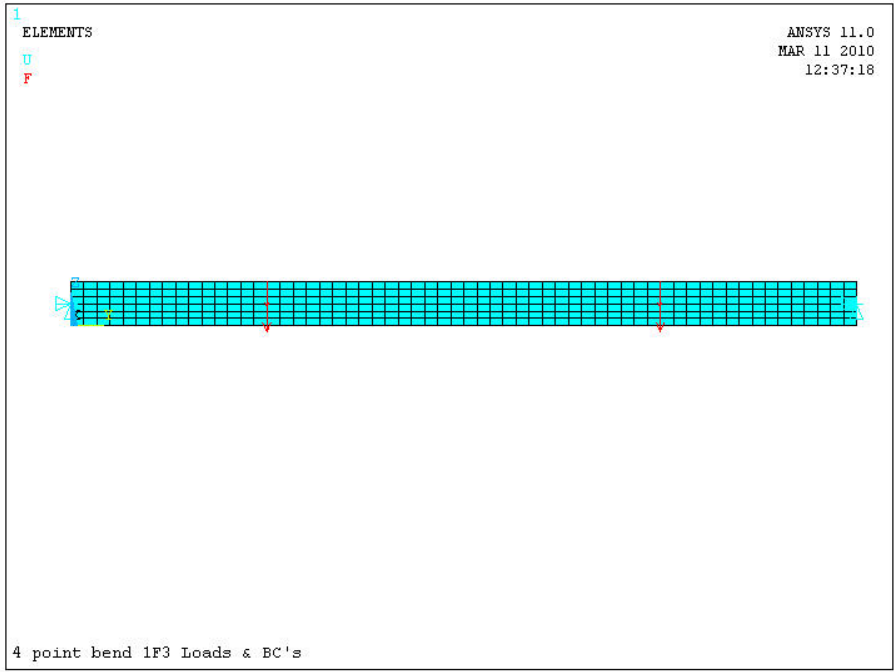

Figure AU-18: Loads and boundary conditions in the FEA model for coupon 1F3.

A sample of the stress distribution in the model for the case of a total flexural load of $50 \mathrm{~N}$ is shown in Figure AU-19. Also, stress and strain distributions at a section in the middle of the support span are shown in the figure to illustrate their values at the outer layer of the cross section and compare to the FBG sensor measurements. 
Intelligent Fiber Optic Sy stems Corp. (IFOS) DOE STTR GRANT No.: DE-

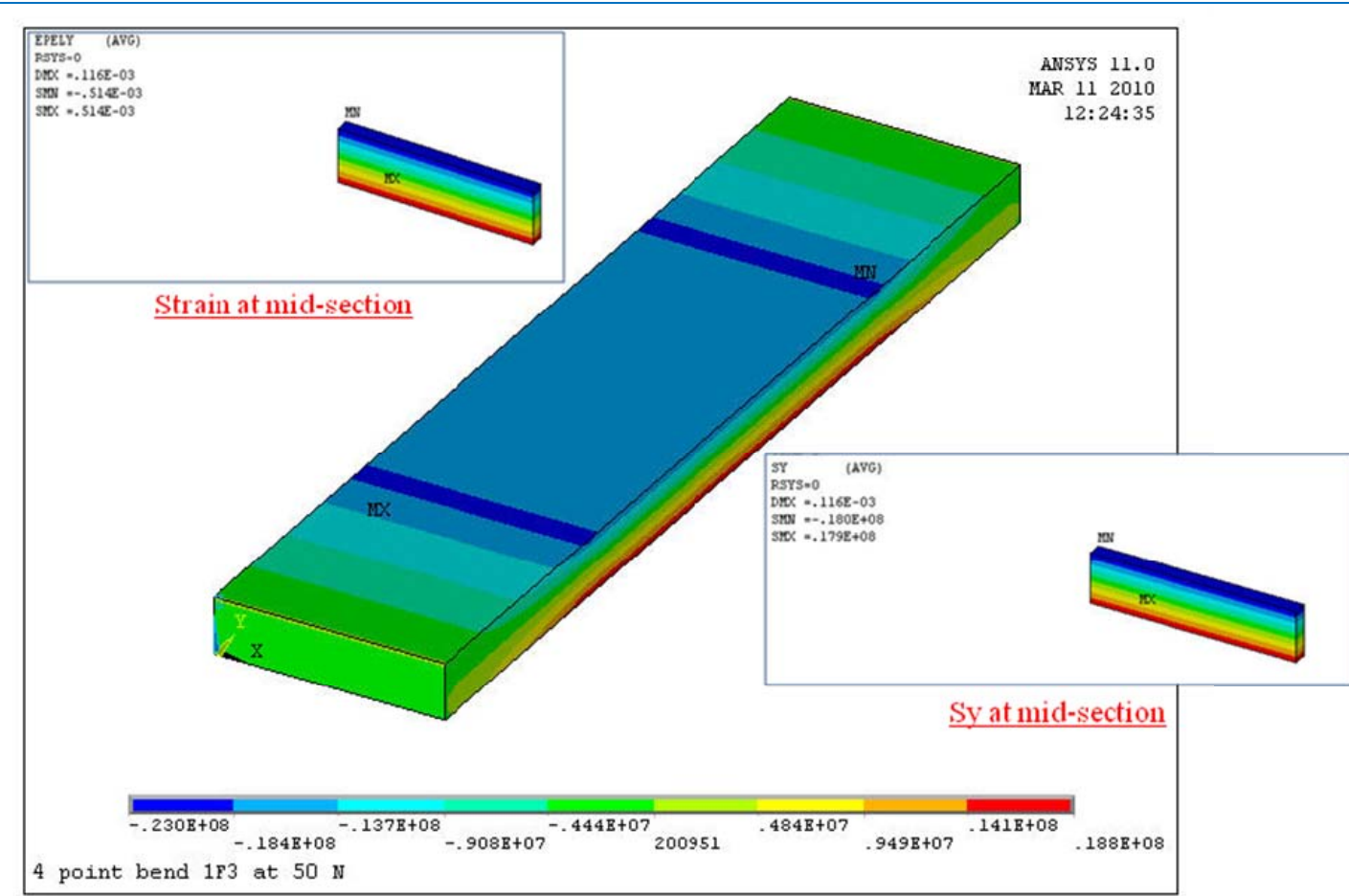

Figure AU-19: Distribution of stress component $S_{\mathrm{y}}$ for the case of a total flexural load of $50 \mathrm{~N}$ in the FEA model for coupon 1 F3.

Figure AU-20 shows strain values measured by both FBG sensors (I-Sense) and Instron output for the outer layer of the coupons at mid-span plotted against the total applied flexural loads. Also, the Figure shows results for the FEA model for coupon $1 \mathrm{~F} 3$.

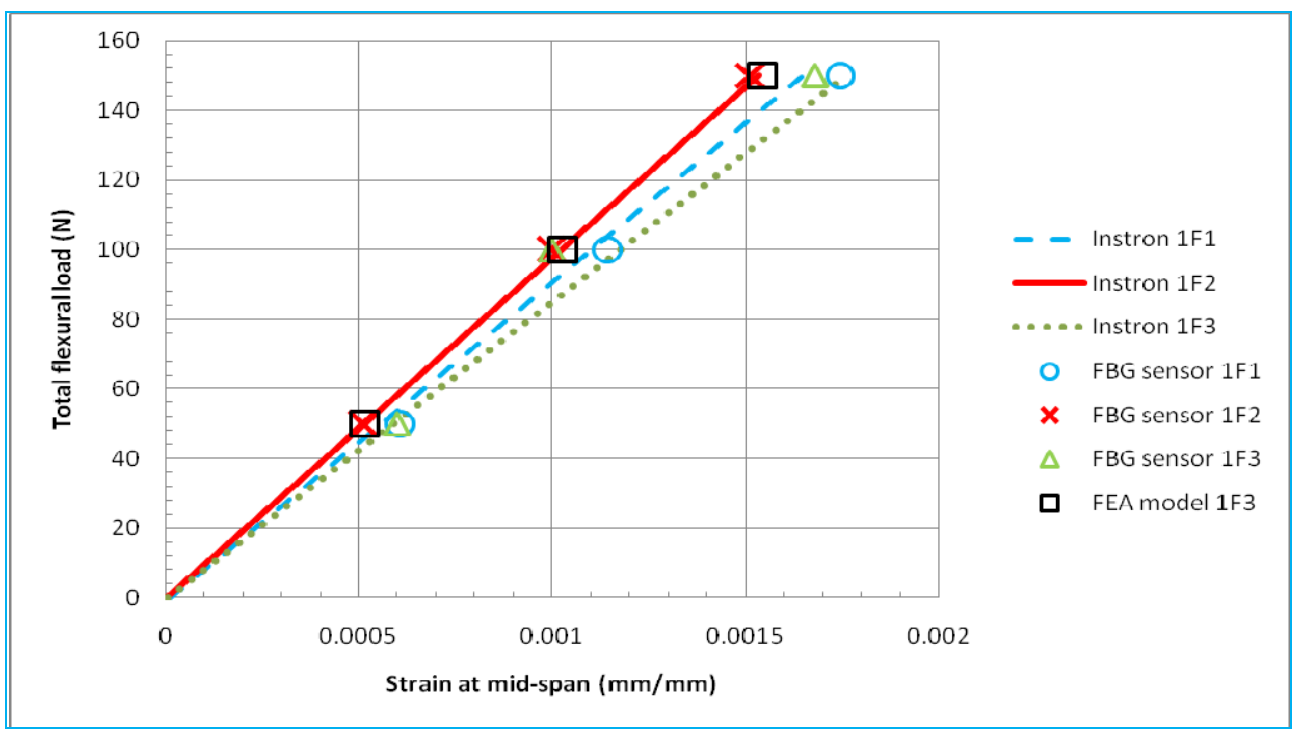

Figure AU-20: Strain at mid-span vs. applied flexural loads for the fourpoint bending tests. 
Intelligent Fiber Optic Sy stems Corp. (IFOS) DOE STTR GRANT No.: DE-

SC0001020

Interim Report \#4

Again, as can be seen in the Figure, experimental and FEA model results reasonably matched at such a low strain level.

\subsubsection{Results of dynamic tests}

4.1.3.1 Impact, vibration, and end-displacement tests

For each test performed, the amplitude and the phase of the transfer function was recorded, see Figures AU-21 to AU-34. From these figures, the frequencies at which peaks occurred were noted and recorded in table AU-3. Since the same frequency could be noted from different figures, the number of occurrence of each frequency was also recorded.

Table AU-3: Frequency at peaks

\begin{tabular}{|c|c|}
\hline Frequency & No. of occurrence \\
\hline $\mathbf{2 4}$ & $\mathbf{1}$ \\
\hline 32 & $\mathbf{1}$ \\
\hline 48 & $\mathbf{2}$ \\
\hline $\mathbf{5 6}$ & $\mathbf{5}$ \\
\hline 72 & 5 \\
\hline 80 & 4 \\
\hline 352 & 3 \\
\hline 376 & 4 \\
\hline 384 & $\mathbf{1}$ \\
\hline 392 & $\mathbf{1}$ \\
\hline 1032 & $\mathbf{1}$ \\
\hline 1040 & $\mathbf{1}$ \\
\hline 1056 & 3 \\
\hline
\end{tabular}

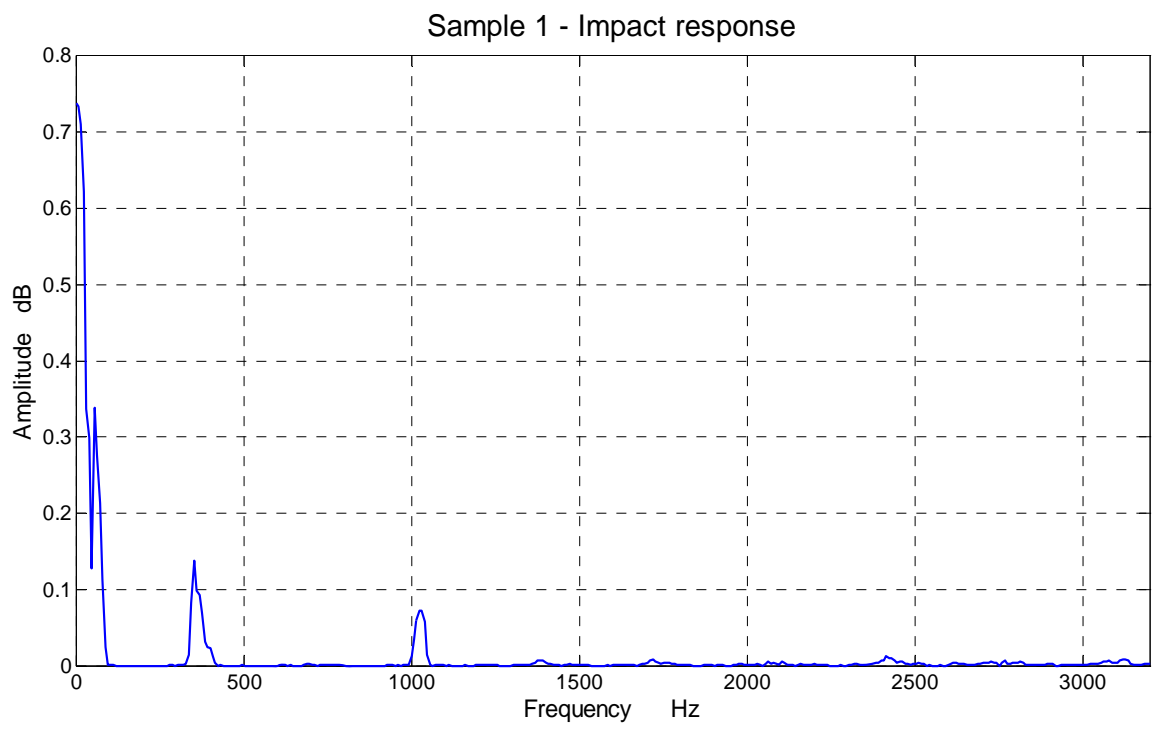

Figure AU-21: Sample 1D1 impact amplitude against frequency 
Intelligent Fiber Optic Sy stems Corp. (IFOS) DOE STTR GRANT No.: DESC0001020

Interim Report \#4

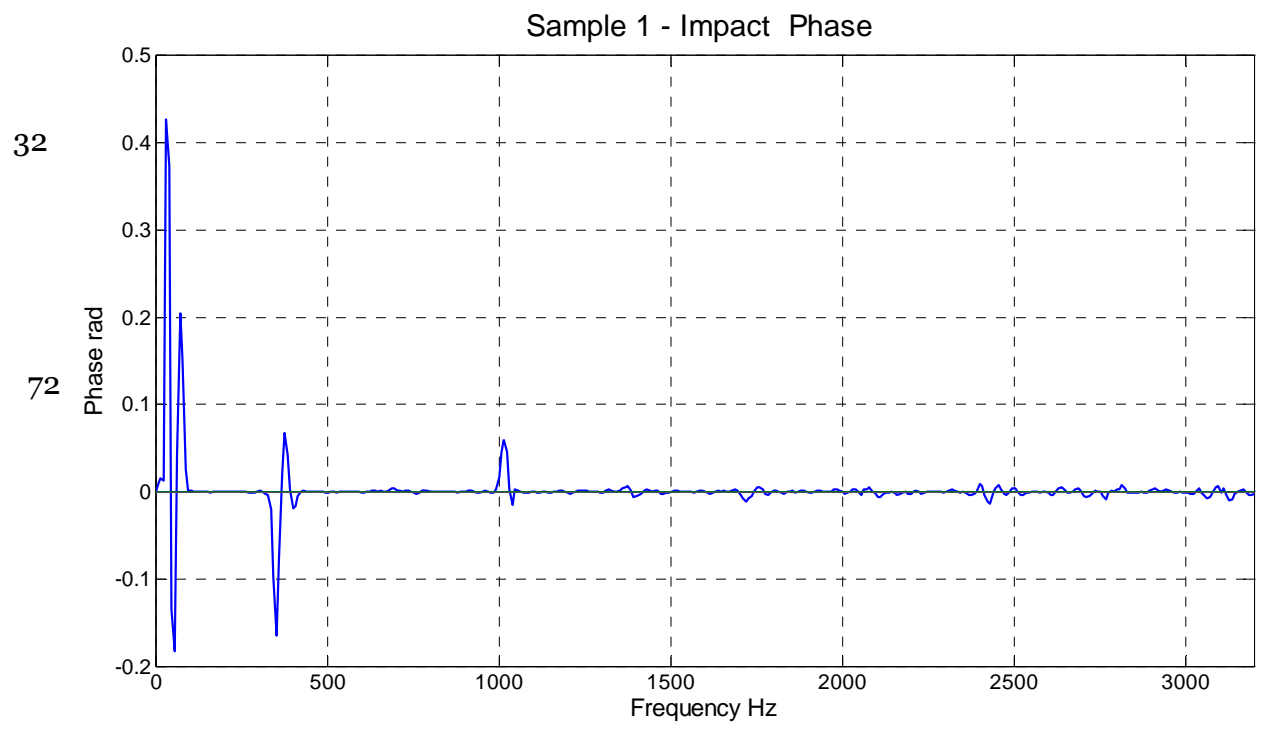

Figure AU-22: Sample 1D1 impact phase against frequency

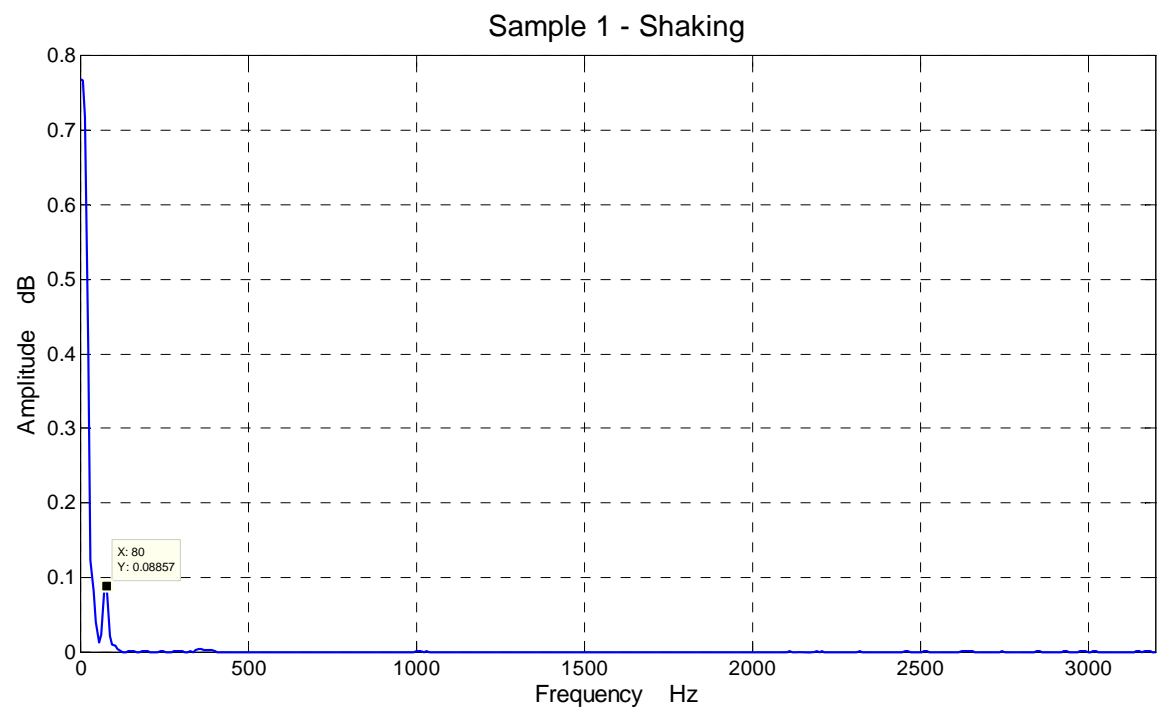

Figure AU-23: Sample 1D1 vibration amplitude against frequency 
Intelligent Fiber Optic Sy stems Corp. (IFOS) DOE STTR GRANT No.: DESC0001020

Interim Report \#4

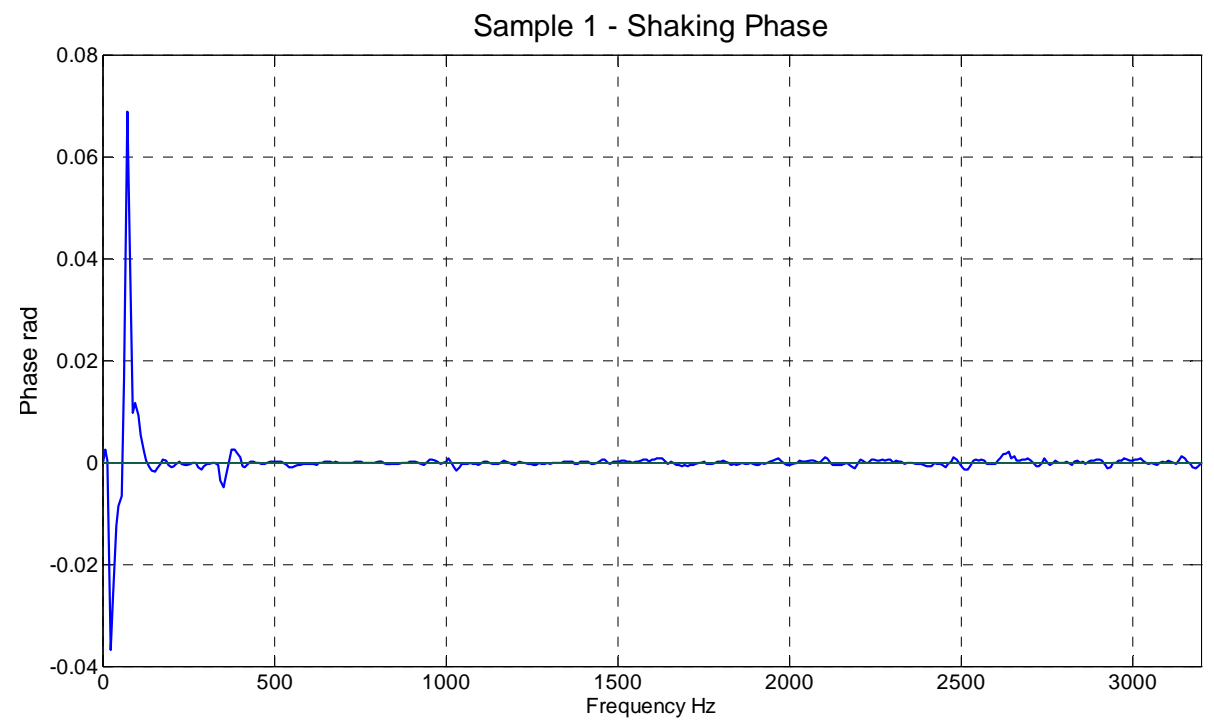

Figure AU-24: Sample 1D1 vibration phase against frequency

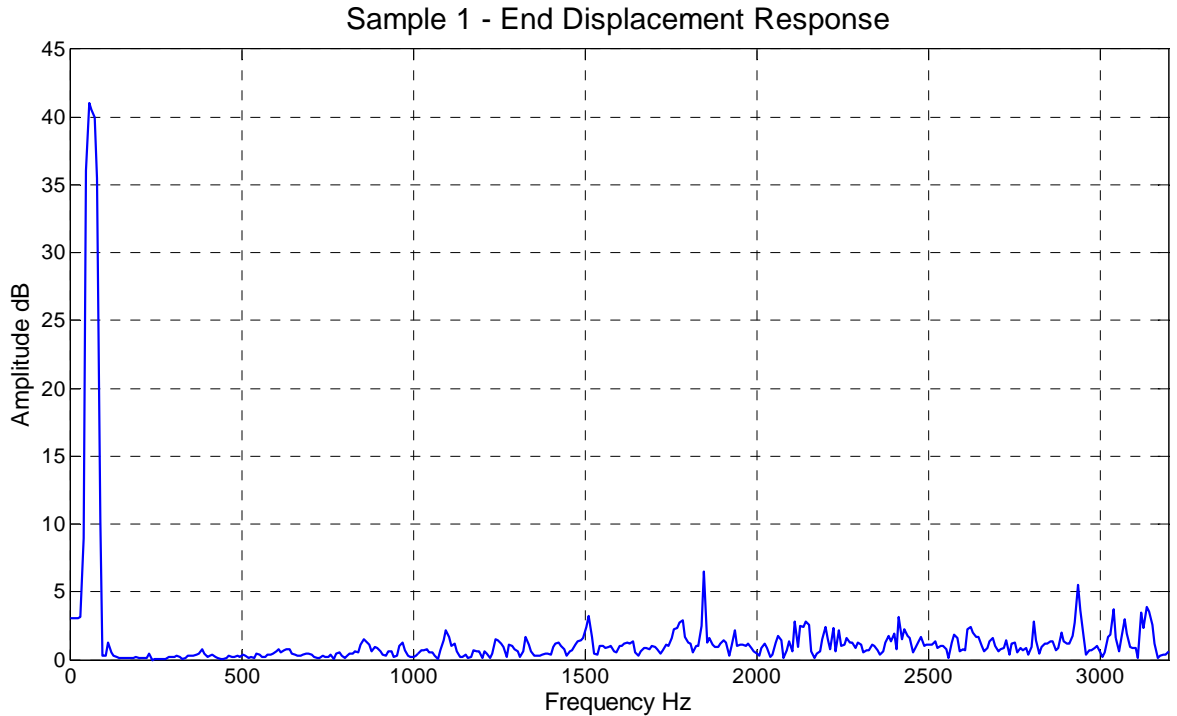

Figure AU-25: Sample 1D1 end displacement amplitude against frequency 
Intelligent Fiber Optic Sy stems Corp. (IFOS) DOE STTR GRANT No.: DE-

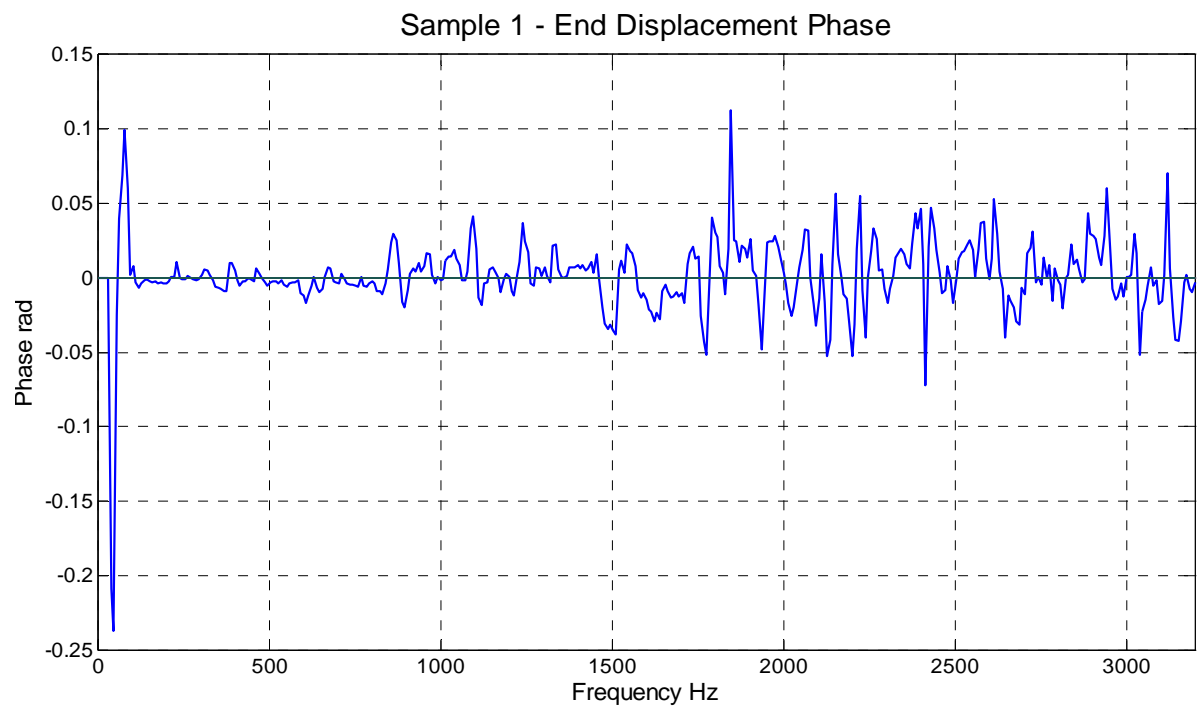

Figure AU-26: Sample 1D1 end displacement phase against frequency

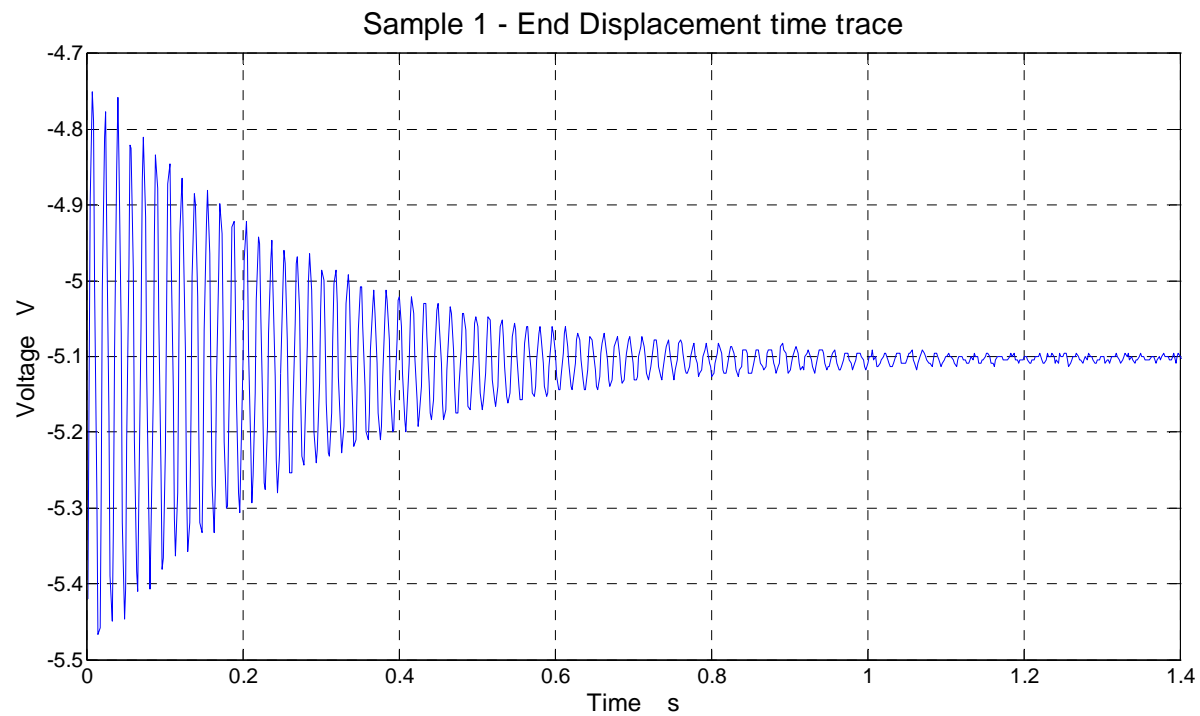

Figure AU-27: Sample 1D1 end displacement time trace 
Intelligent Fiber Optic Sy stems Corp. (IFOS) DOE STTR GRANT No.: DESC0001020

Interim Report \#4

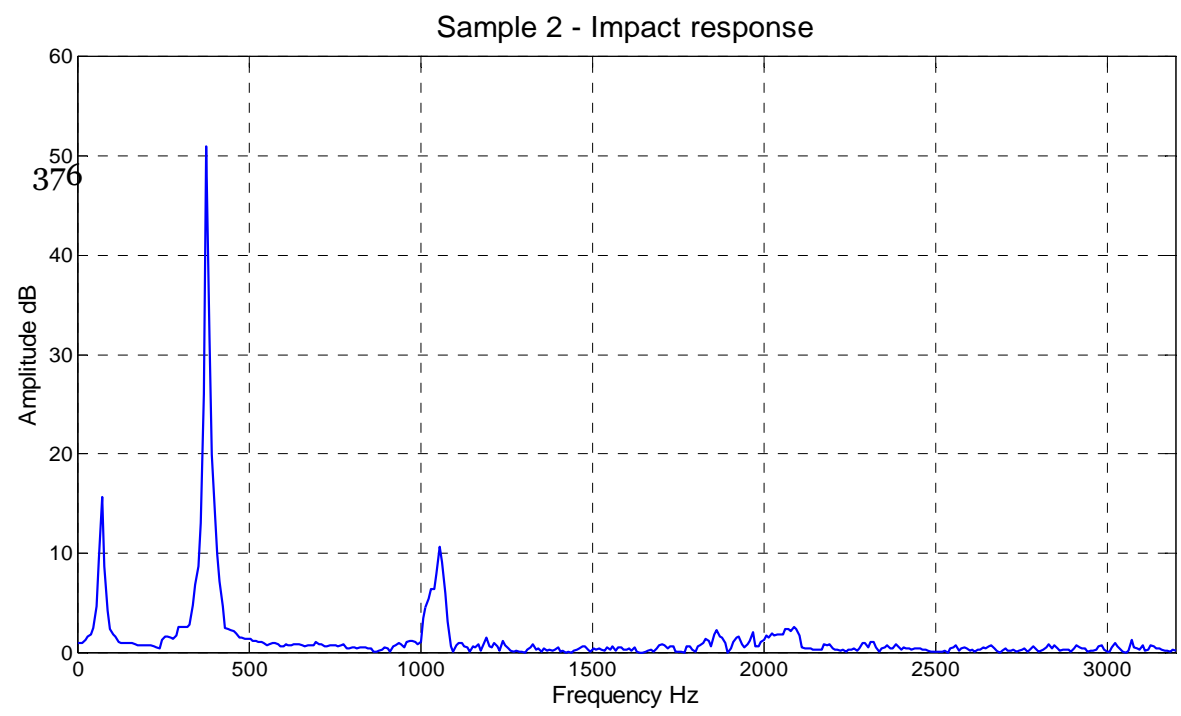

Figure AU-28: Sample 1D3 impact amplitude against frequency

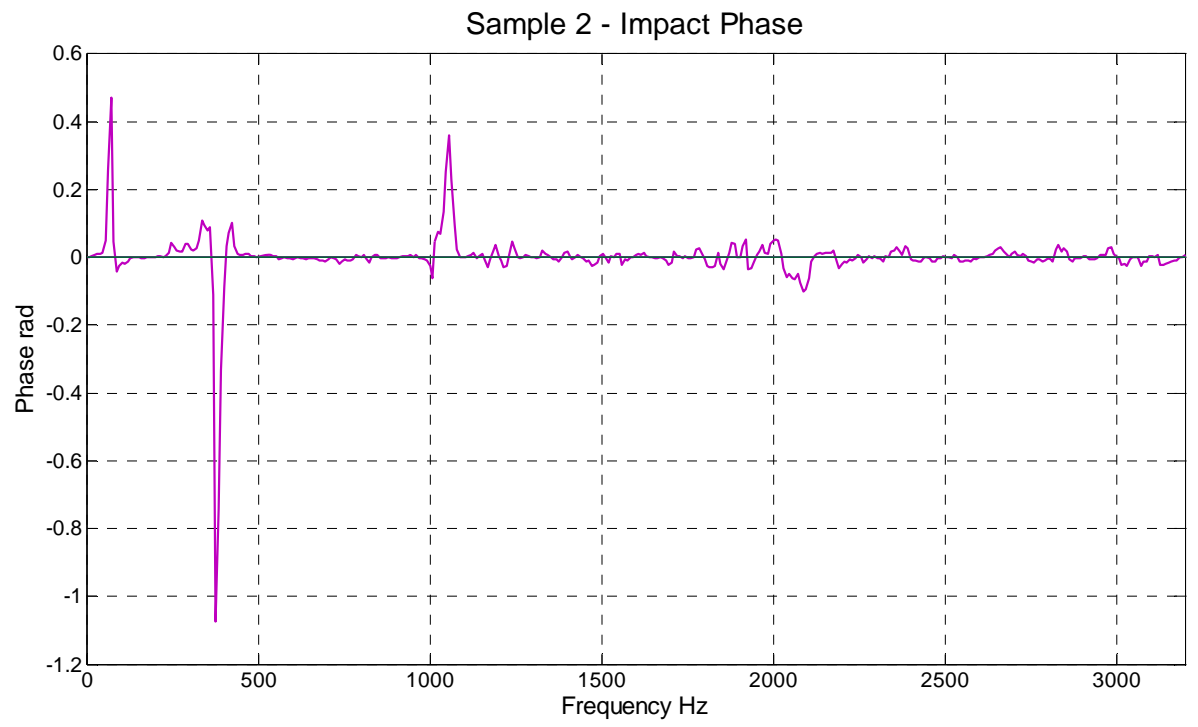

Figure AU-29: Sample 1D3 impact phase against frequency 
Intelligent Fiber Optic Sy stems Corp. (IFOS) DOE STTR GRANT No.: DE-

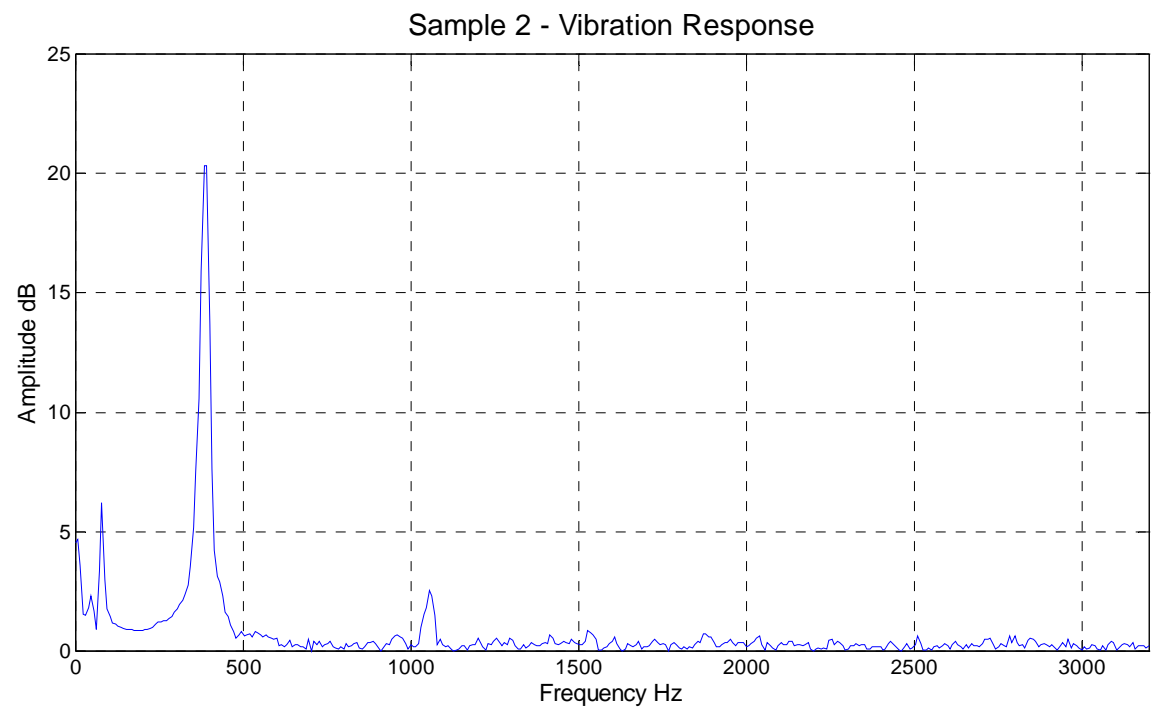

Figure AU-30: Sample 1D3 vibration amplitude against frequency

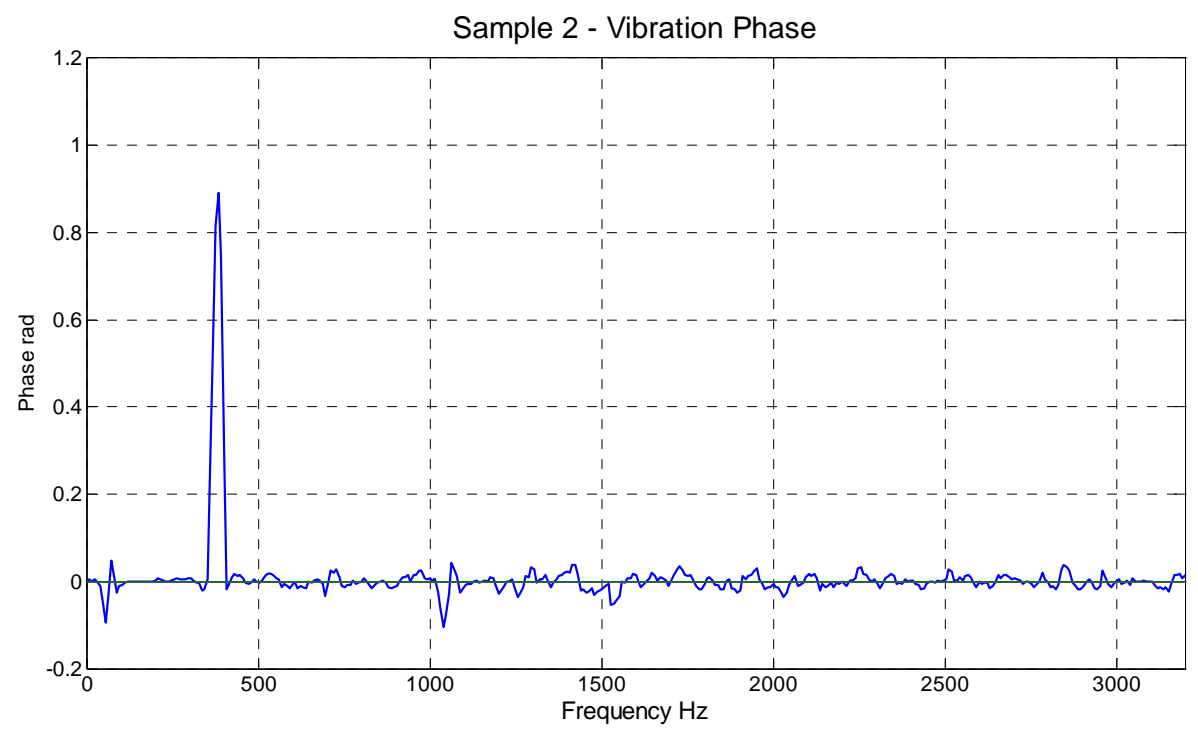

Figure AU-31: Sample 1D3 vibration phase against frequency 
Intelligent Fiber Optic Sy stems Corp. (IFOS) DOE STTR GRANT No.: DESC0001020

Interim Report \#4

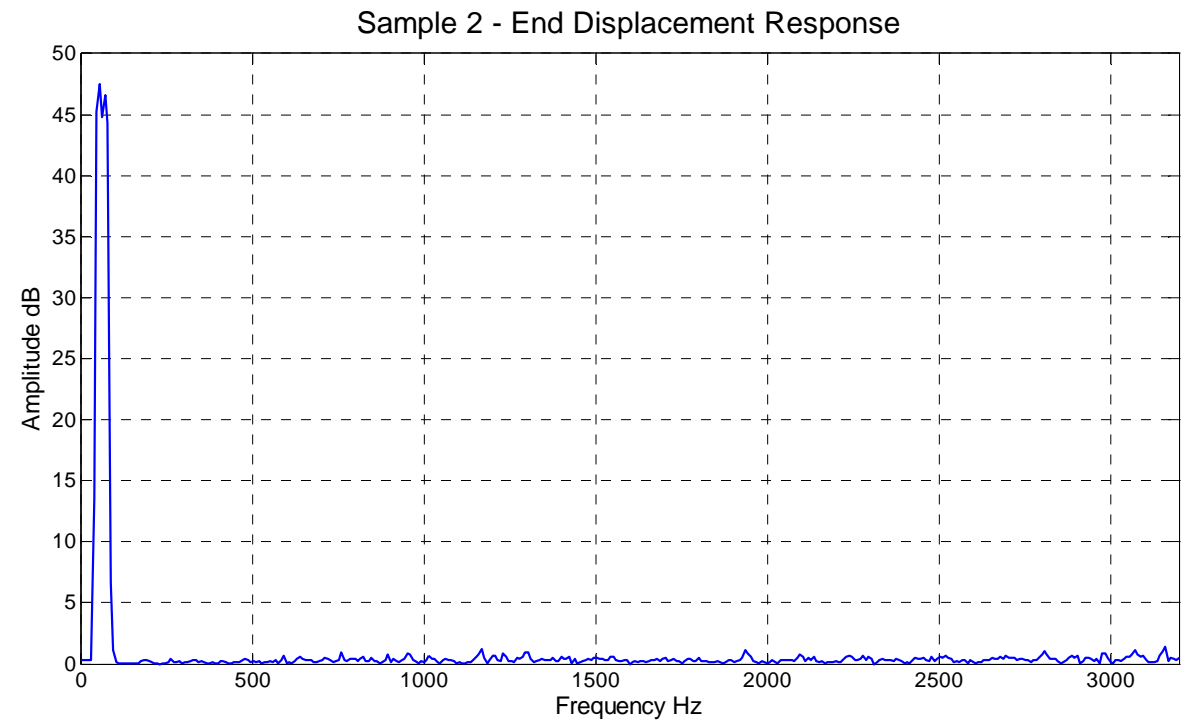

Figure AU-32: Sample 1D3 end displacement amplitude against frequency

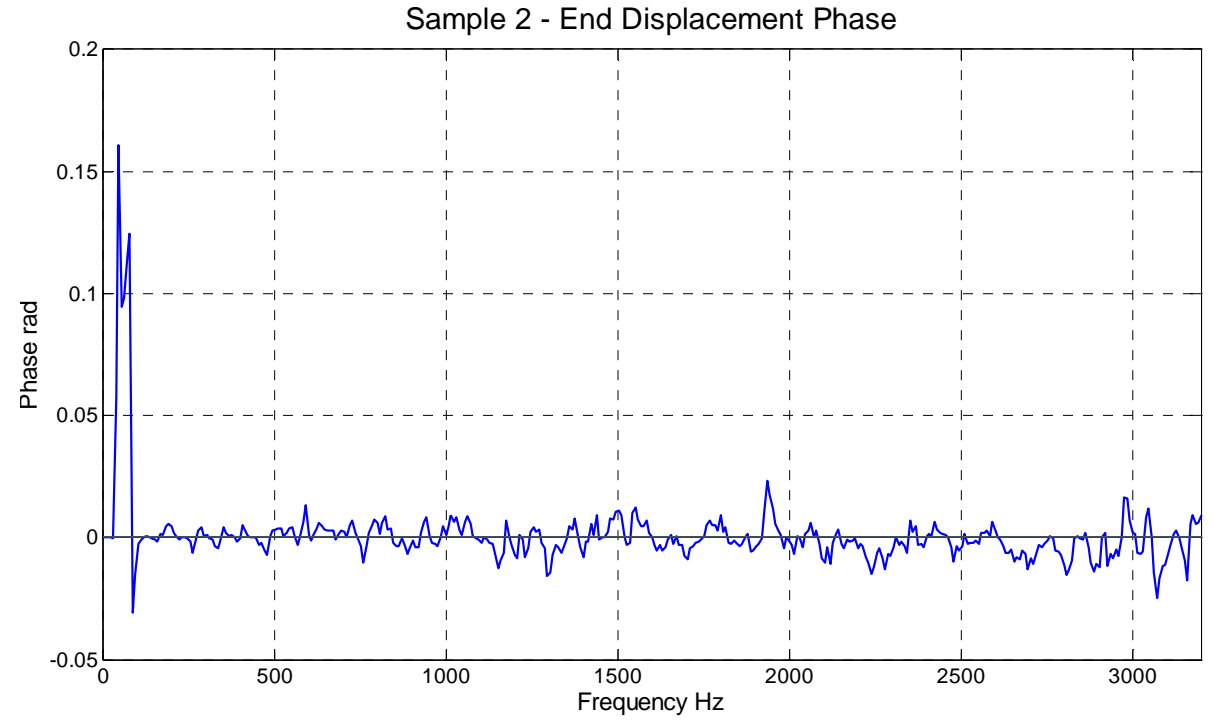

Figure AU-33: Sample 1D3 end displacement phase against frequency 
Intelligent Fiber Optic Sy stems Corp. (IFOS) DOE STTR GRANT No.: DE-

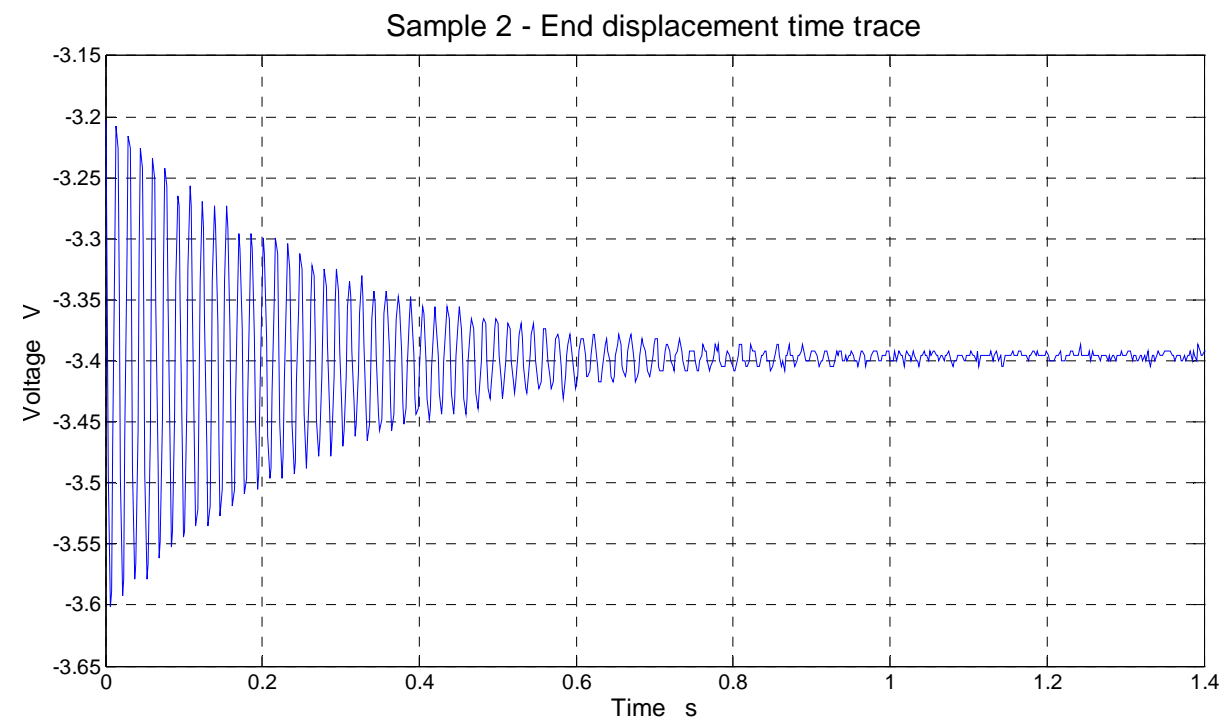

Figure AU-34: Sample 1D3 end displacement time trace

\subsubsection{Modal analysis for FEA model}

A finite element model was built using ANSYS for coupon 1D1. Elastic properties of the model were the same as those in Table AU-2. The model was meshed into 3600 elements using the "SOLID 95" element type. Boundary conditions were taken as those of a clamped-free bar. A modal analysis was performed to determine the mode shapes and natural frequencies of the model. Figure AU-35 shows the first five mode shapes of the model. Natural frequencies of the model are listed in Table AU-4. 
Intelligent Fiber Optic Sy stems Corp. (IFOS) DOE STTR GRANT No.: DE-

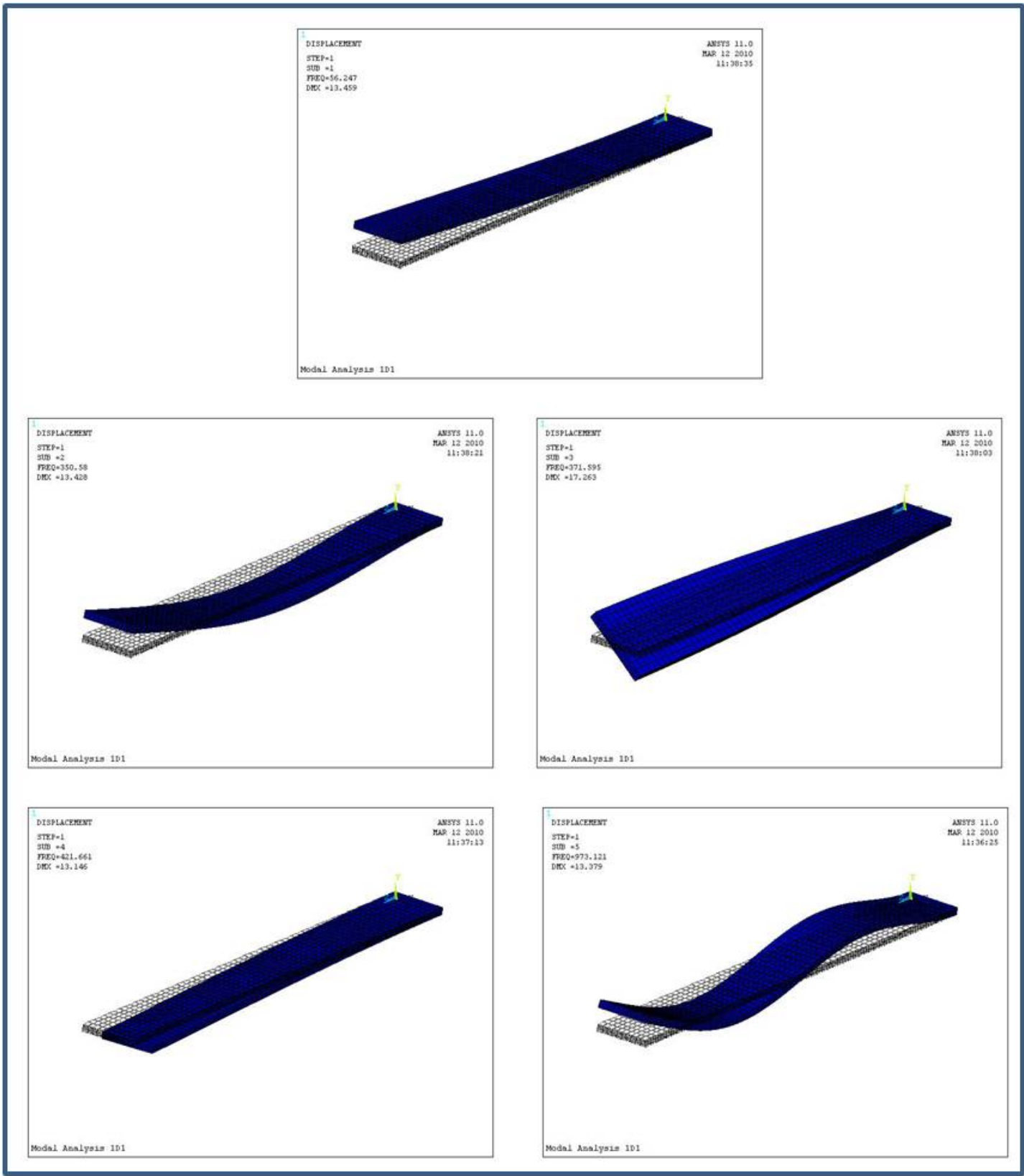

Figure AU-35: Mode shapes for FEA model of coupon $1 \mathrm{D} 1$.

Table AU-4: Natural frequencies for FEA model of coupon $1 D 1$

\begin{tabular}{|c|c|}
\hline Mode & $\begin{array}{c}\text { Natural frequency } \\
(\mathrm{Hz})\end{array}$ \\
\hline 1 & 56.247 \\
\hline 2 & 350.58 \\
\hline 3 & 371.59 \\
\hline 4 & 421.66 \\
\hline 5 & 973.12 \\
\hline
\end{tabular}


Intelligent Fiber Optic Sy stems Corp. (IFOS) DOE STTR GRANT No.: DE-

Results of the modal analysis show agreement with those of the dynamic tests as can be seen from the matching values of natural frequencies listed in Tables AU-3 and AU-4.

\subsubsection{Strain measurements in end-displacement tests}

Three sets of end-displacement tests were performed on coupons 1D1 and 1D3. In each of these tests, the free end of the clamped coupon was subjected to an initial vertical displacement, as shown in Figure AU-36, and then released to vibrate freely. The transient response of the coupon was recorded from both the FBG sensor using the Mini I-sense software and the output vibrometer connected to the analyzer.

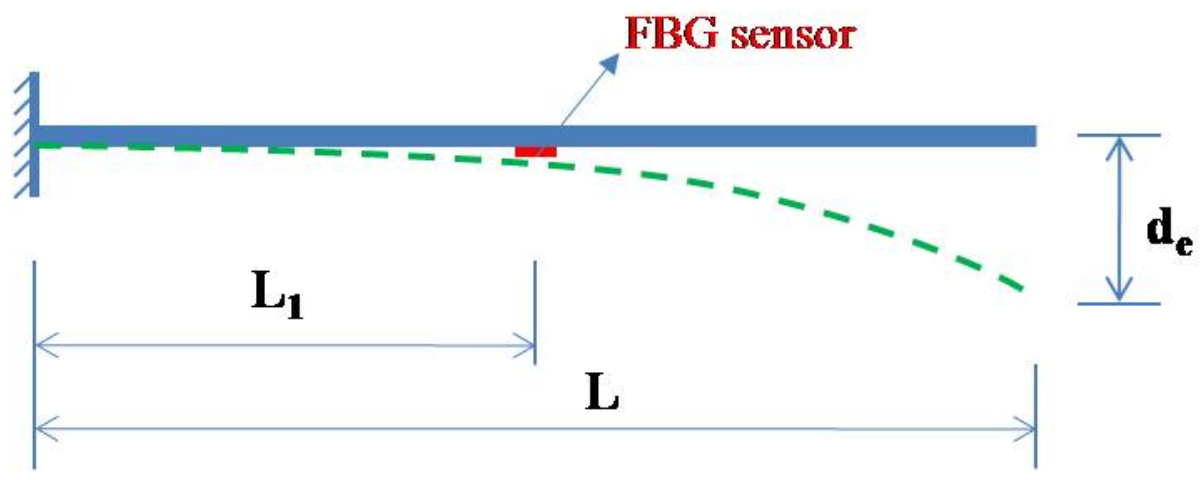

Figure AU-36: Schematic diagram for the end-displacement tests.

Measurements recorded using the Mini I-sense software for the corresponding strain values at the position of the mounted FBG sensors are shown for coupons 1D1 and 1D3 in Figures AU-37 and AU-38, respectively. 
Intelligent Fiber Optic Sy stems Corp. (IFOS) DOE STTR GRANT No.: DE-

Interim Report \#4

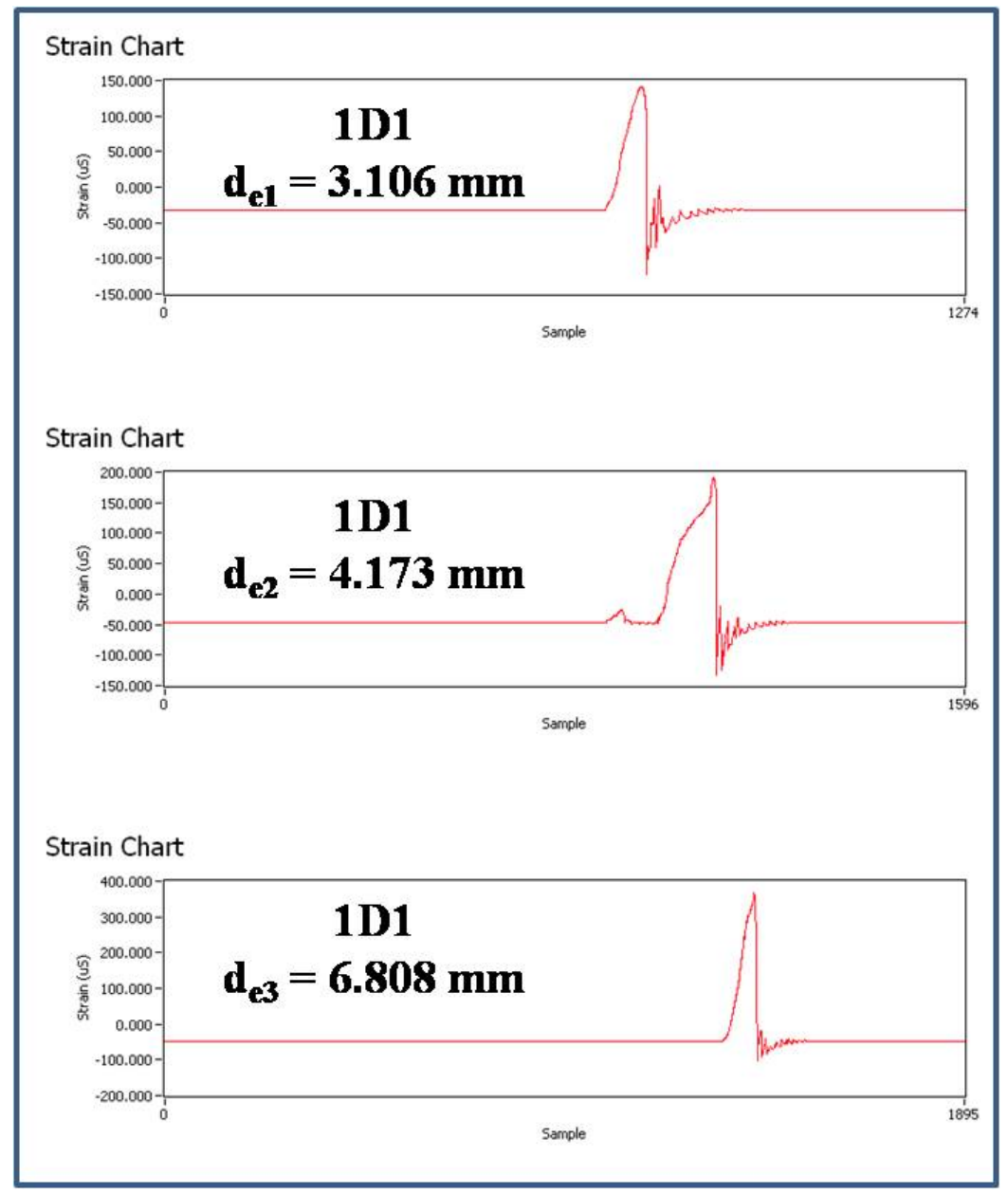

Figure AU-37: Strain measurements at FBG sensor position in the end displacement tests of coupon $1 D 1$. 
Intelligent Fiber Optic Sy stems Corp. (IFOS) DOE STTR GRANT No.: DE-

Interim Report \#4

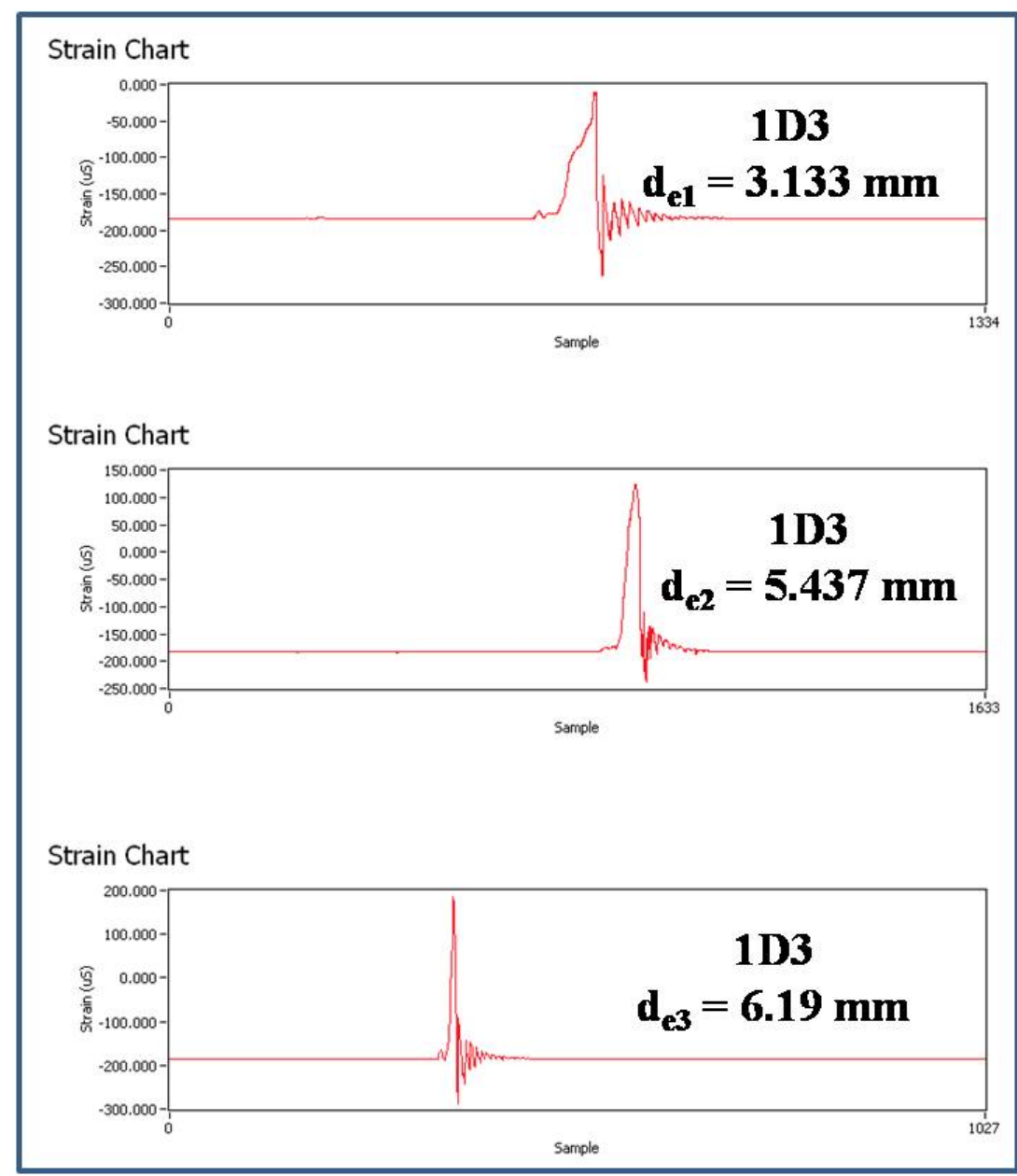

Figure AU-38: Strain measurements at FBG sensor position in the end displacement tests of coupon $1 D 3$.

Also, time trace recorded for the end displacements of the coupons by the output vibrometer are shown in Figures AU-39 and AU-40 for coupons 1D1 and 1D3, respectively. Maximum values of recorded downward and upward end displacements were obtained from the time trace data and listed in Table AU-5 for both coupons. 
Intelligent Fiber Optic Sy stems Corp. (IFOS) DOE STTR GRANT No.: DE-

Interim Report \#4
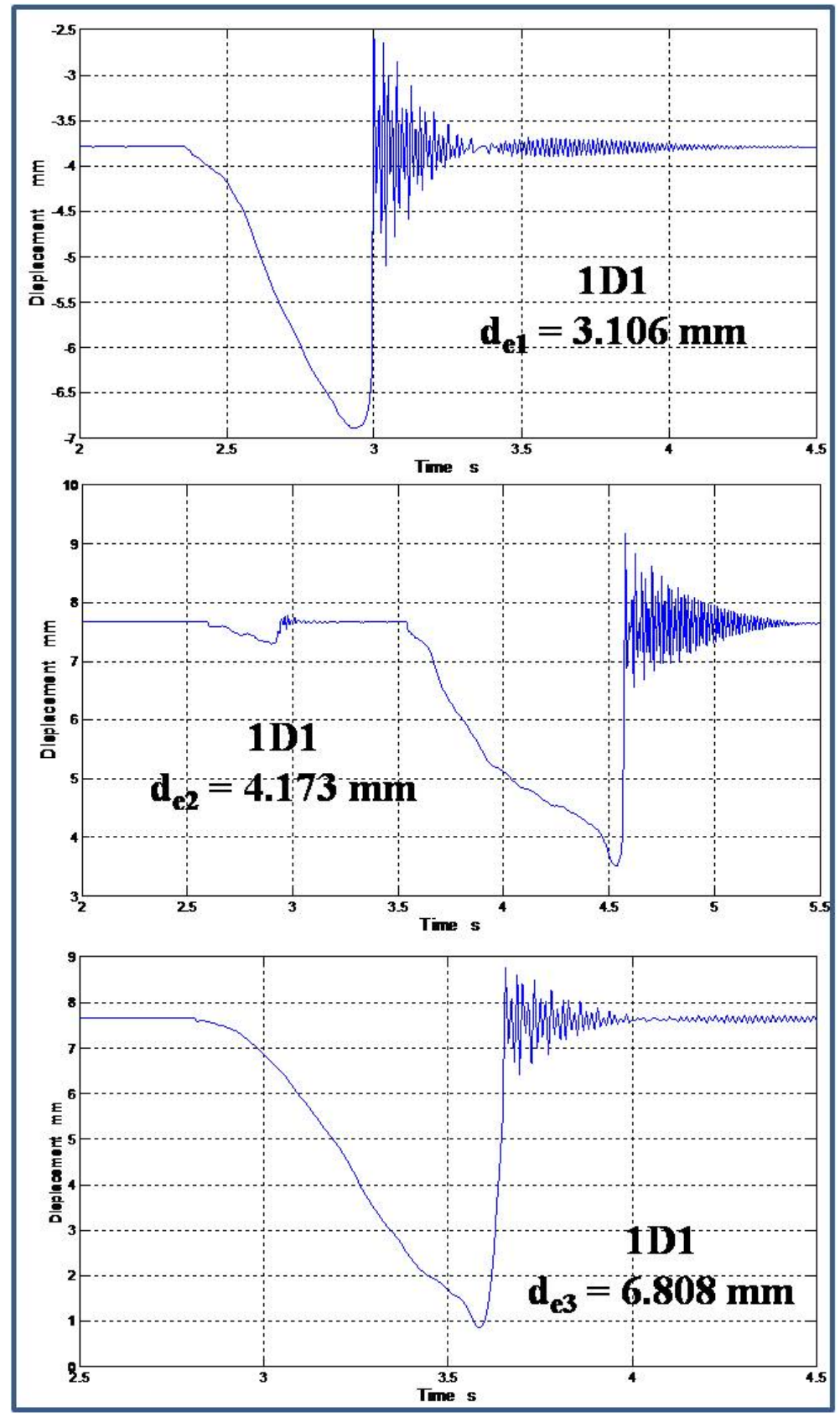

Figure AU-39: Time trace for end displacement tests of coupon $1 D 1$. 
Intelligent Fiber Optic Sy stems Corp. (IFOS) DOE STTR GRANT No.: DESC0001020

Interim Report \#4

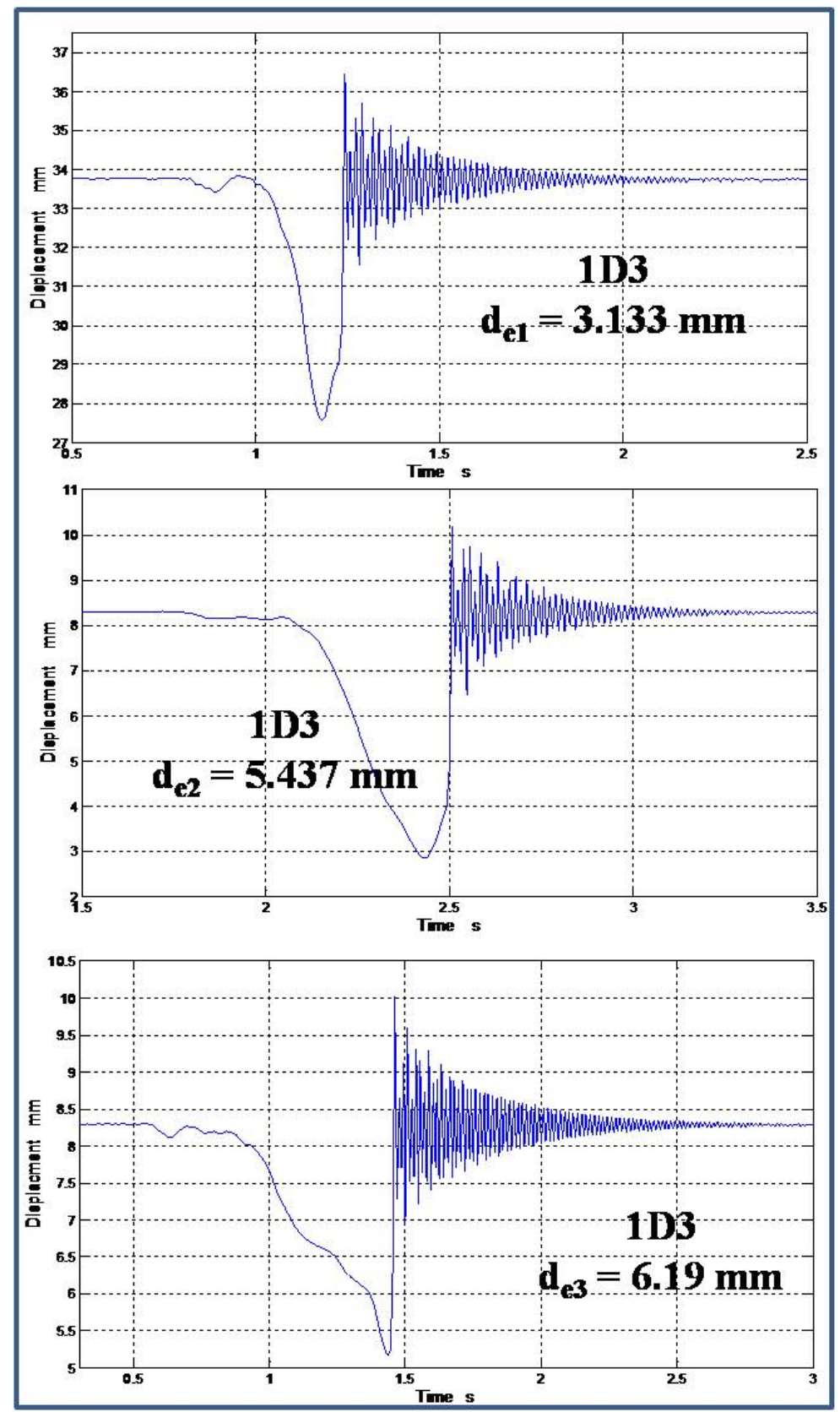

Figure AU-40: Time trace for end displacement tests of coupon $1 D 3$.

Table AU-5: Maximum recorded end displacements at free end.

\begin{tabular}{|c|c|c|c|c|}
\hline \multirow[t]{2}{*}{ Test } & \multicolumn{2}{|c|}{ Coupon 1D1 } & \multicolumn{2}{|c|}{ Coupon $1 D 3$} \\
\hline & $\begin{array}{c}\text { Max. } \\
\text { disp down }\end{array}$ & $\begin{array}{c}\text { Max. } \\
\text { disp up }\end{array}$ & $\begin{array}{c}\text { Max. } \\
\text { disp down }\end{array}$ & $\begin{array}{l}\text { Max. } \\
\text { disp up }\end{array}$ \\
\hline$d_{\text {e1 }}$ & 3.106 & -1.179 & 3.133 & -1.715 \\
\hline $\mathbf{d e}_{\mathrm{e} 2}$ & 4.173 & -1.498 & 5.437 & -1.889 \\
\hline$d_{\text {e3 }}$ & 6.808 & -1.118 & 6.19 & -2.7 \\
\hline
\end{tabular}


Intelligent Fiber Optic Sy stems Corp. (IFOS) DOE STTR GRANT No.: DE-

Using the principles of the Beam Theory as a reasonable approximation, the relation between the end-displacement and the corresponding strain at the position of the mounted FBG sensor can be analytically derived to be:

$$
\mathrm{S}_{\mathrm{L} 1}=1.5 * \mathrm{t} * \mathrm{~d}_{\mathrm{e}} *\left(\mathrm{~L}-\mathrm{L}_{1}\right) / \mathrm{L}^{3}
$$

Where $\mathbf{S}_{\mathbf{L} 1}$ is the strain at position of FBG sensor corresponding to an end displacement $\mathbf{d}_{\mathbf{e}}, \mathbf{L}$ is the span of the clamped coupon, $\mathbf{L}_{\mathbf{1}}$ is the distance of the FBG sensor from the clamped end, and $\mathbf{t}$ is the thickness of the coupon.

Strain values corresponding to the maximum downward and upward end displacements, listed above in Table AU-5, were calculated from the above equation. To validate strain measurements of the FBG sensors in these tests, the calculated strain values were compared to those measured and recorded by the FBG sensors as shown in Figures AU-41 and AU-42.

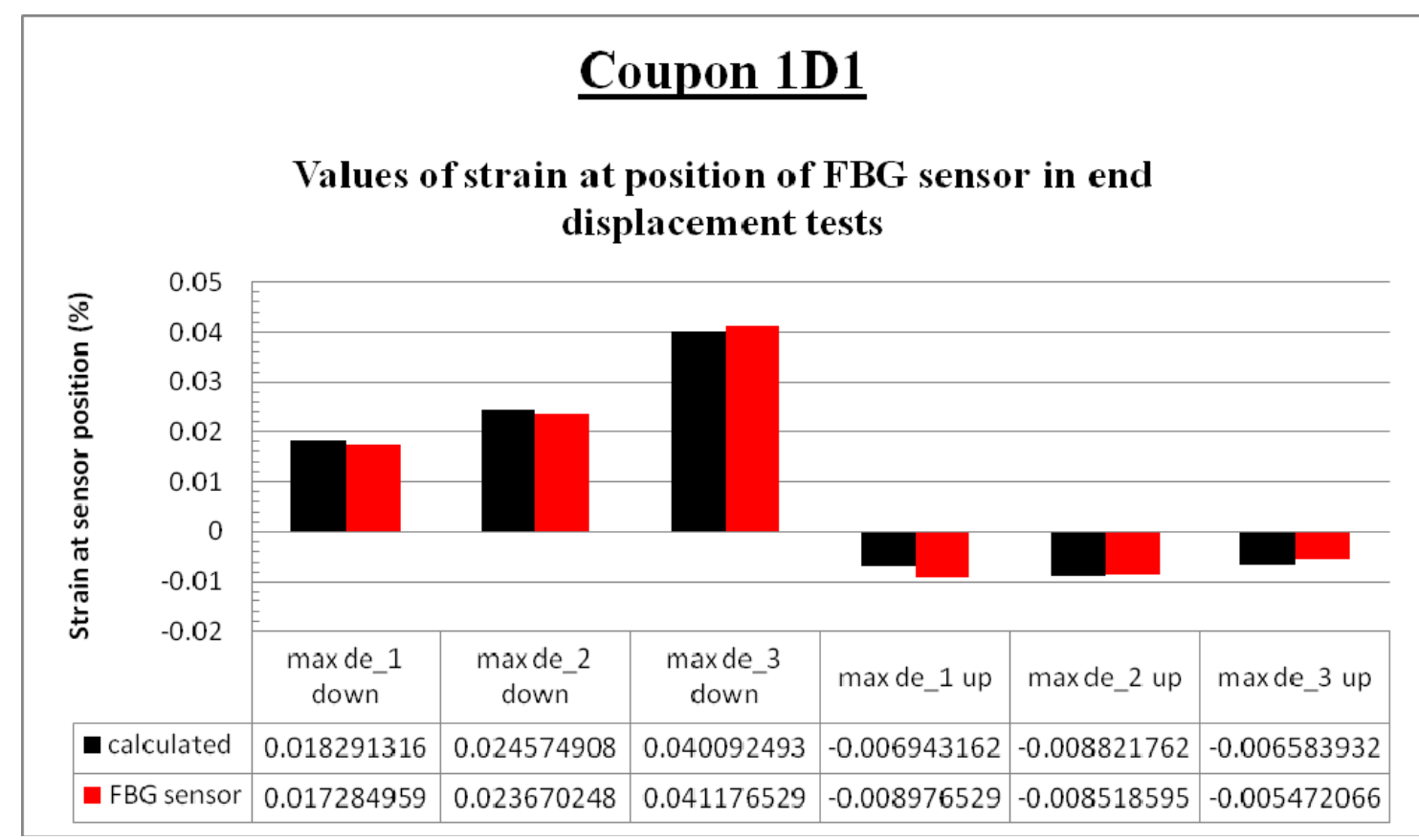

Figure AU-41: Comparison between calculated and measured strain values for end displacement tests of coupon $1 \mathrm{D} 1$. 


\section{Coupon 1D3}

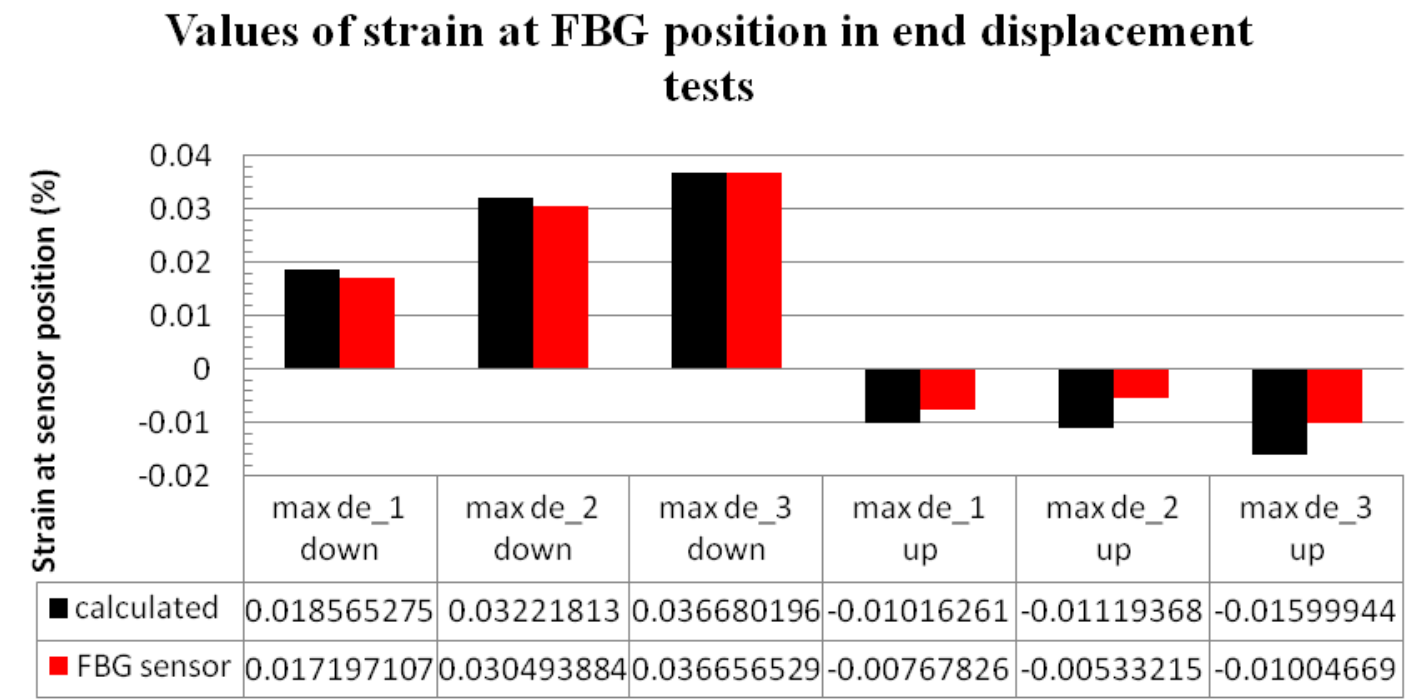

Figure AU-42: Comparison between calculated and measured strain values for end displacement tests of coupon $1 D 3$.

As can be seen in these Figures, accuracy of the FBG sensors is found to be reasonably high for such high strain values.

\subsubsection{Calculation of damping coefficient}

Several runs in ANSYS were conducted on the FEA model for coupon 1D1 to estimate the value of the damping coefficient of the composite. Results of the time trace for the case of the end displacement test, shown in Figure AU-39, were taken as a reference behavior that needs to be matched in these runs. This reference time trace was taken with a starting point at the first maximum upward displacement (which is $1.179 \mathrm{~mm}$, as listed in Table AU- 5). Transient analyses for the model were conducted with the initial condition of $1.179 \mathrm{~mm}$ end displacement with different values of damping coefficients. It was concluded from these runs that the value of 0.00005 for the damping coefficient produced a reasonable match to the reference time trace, as seen in Figure AU-43. 


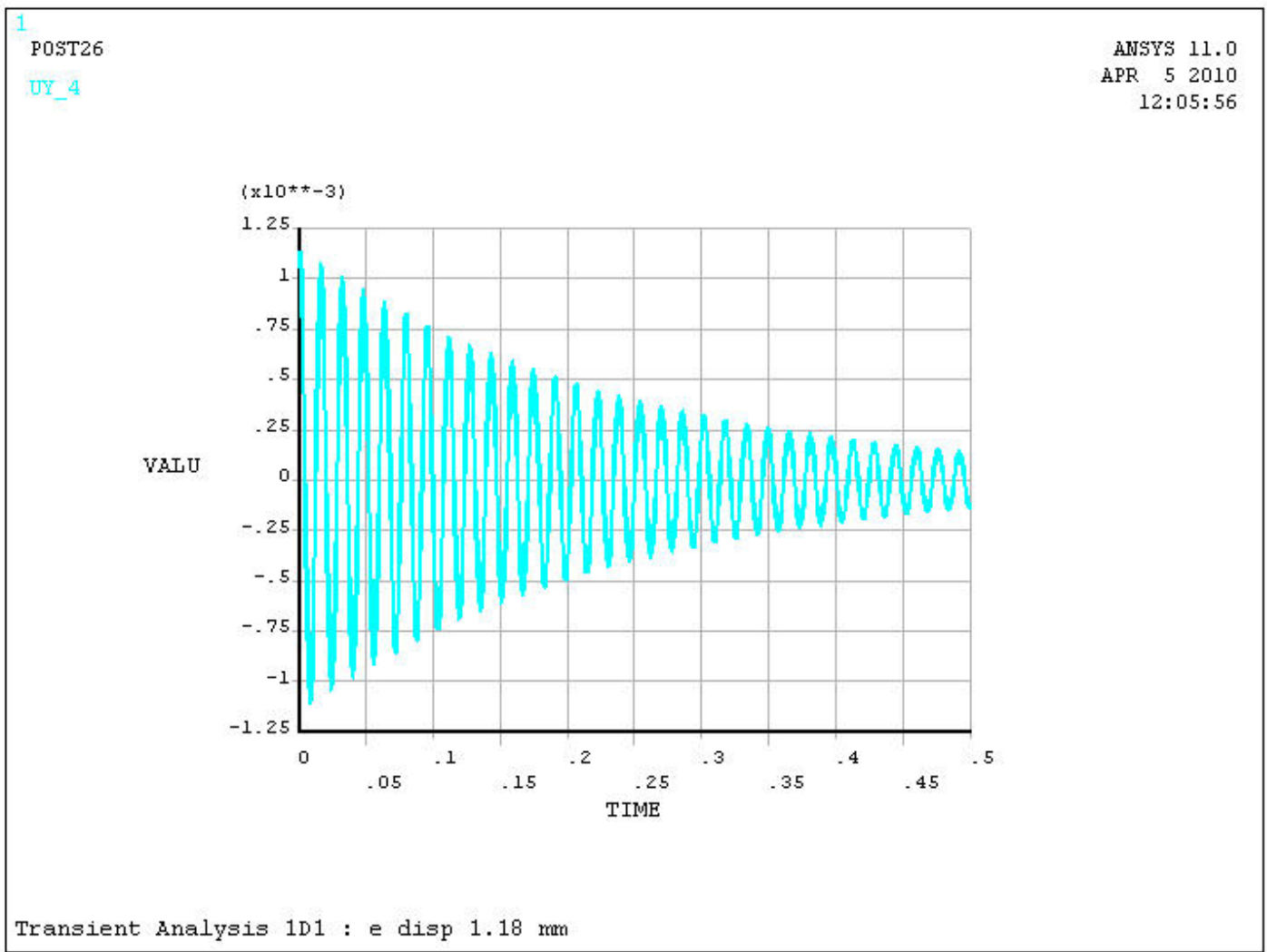

Figure AU-43: Transient response of the vertical displacement $u_{y}$ of a node at the free end of the FEA model of coupon $1 D 1$ with the initial condition of $1.179 \mathrm{~mm}$ upward displacement.

\subsubsection{Results for the effect of defect on natural frequency}

In order to examine the effect of an existing defect on the natural frequency of a composite structure, two coupons from plate 2 were tested. The first coupon was free of defects while the second had two $3 \mathrm{~mm}$ diameter holes with their centers at $35 \mathrm{~mm}$ from each end of the coupon. Also, the second coupon had a delamination between two of its six fabric layers.

Dynamic testing of each of the two coupons was then carried out to determine their natural frequencies, as recorded in Figures AU-44 and AU-45. A shift in the natural frequency is evident between coupons of the two plats validating the hypothesis that strain measurement can be used to detect defects in composite structures. 
Intelligent Fiber Optic Sy stems Corp. (IFOS) DOE STTR GRANT No.: DE-

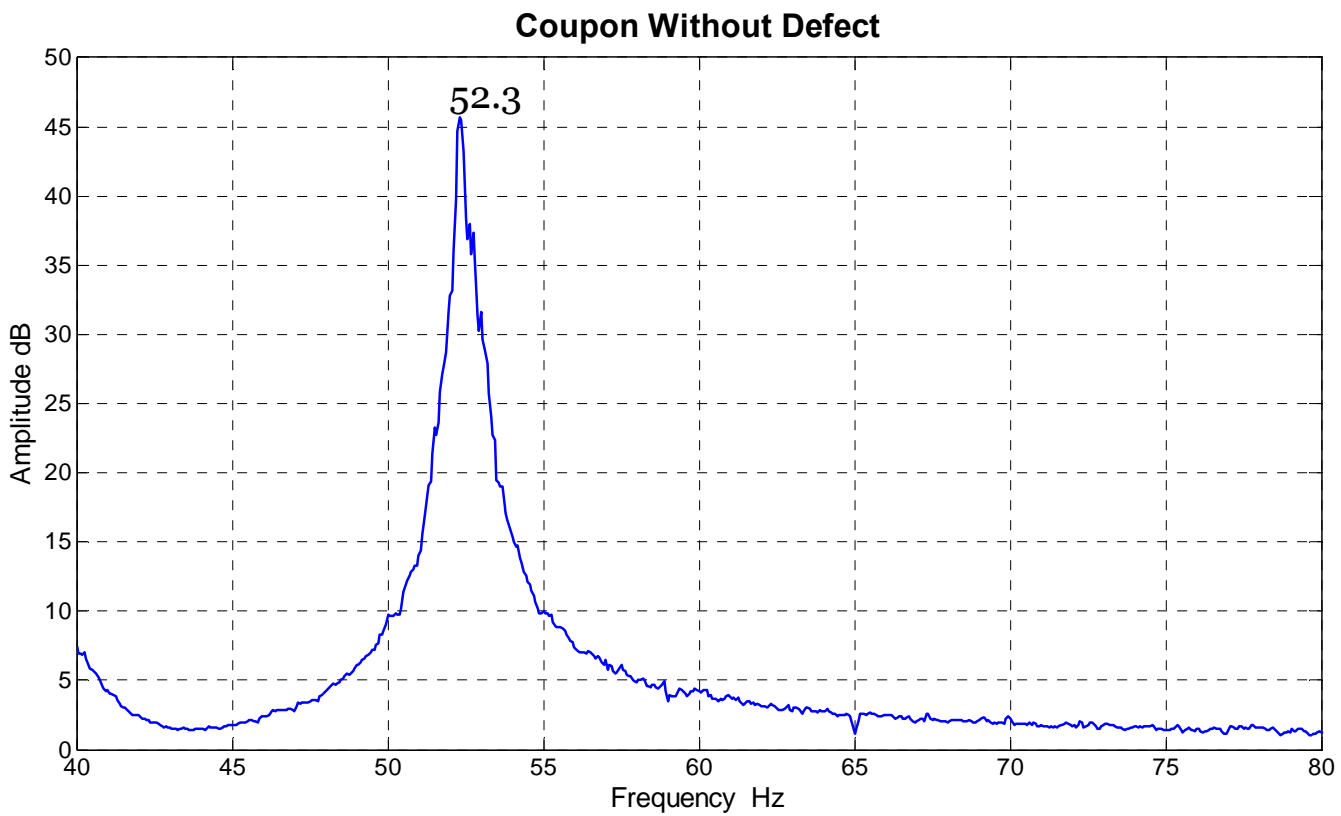

Figure AU-44: Frequency response of composite coupon without defect

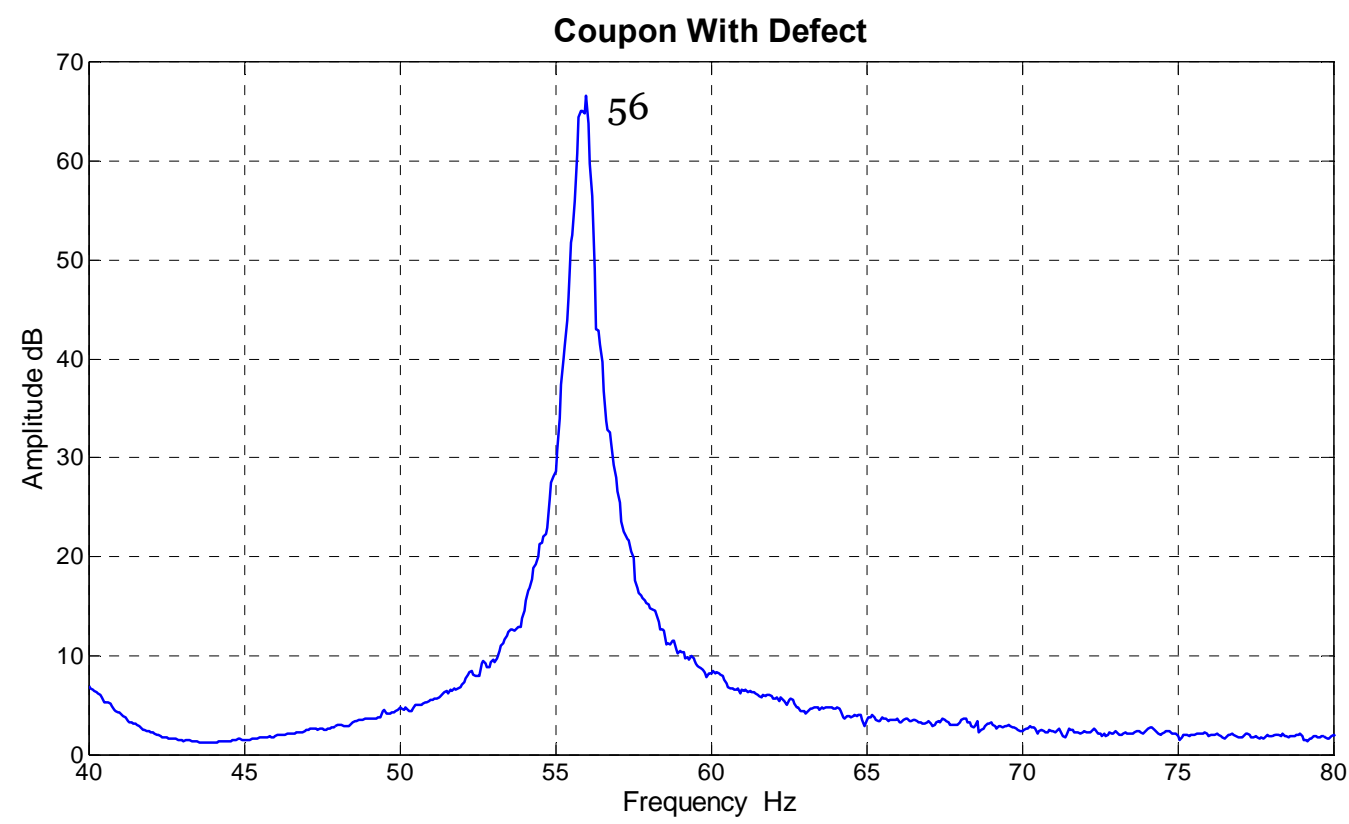

Figure AU-45: Frequency response of composite coupon with defect

\subsubsection{Results of FEA blade model}

A FEA model was built in ANSYS for a wind turbine blade. The NuMAD software was utilized to create the geometric model of the blade. The geometry was chosen from one of the tutorial models in the NuMAD User's Manual. In this model, the blade had a 
Intelligent Fiber Optic Sy stems Corp. (IFOS) DOE STTR GRANT No.: DE-

SC0001020

Interim Report \#4

nominal length of $7.92 \mathrm{~m}$ and its profile was composed of 8 stations (2 with circular cross sections and 6 with airfoil sections with different chord lengths) as shown in Figure AU-46.

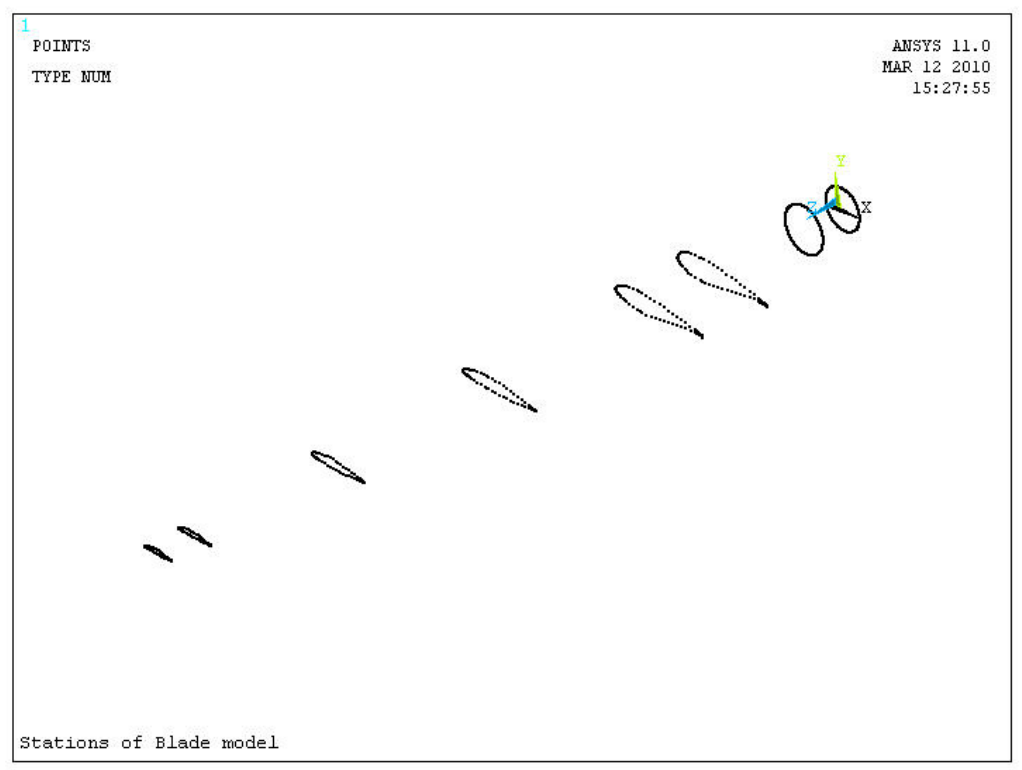

Figure AU-46: Stations of the blade geometry.

The geometric model was incorporated in ANSYS and meshed with 1011 shell elements of type "SHELL99" as shown in Figure AU-47. Mechanical properties of the model were taken as those listed in Table AU-2. Boundary conditions were taken as a clamped-free bar with all fixation constraints applied to the circular cross section at the edge.

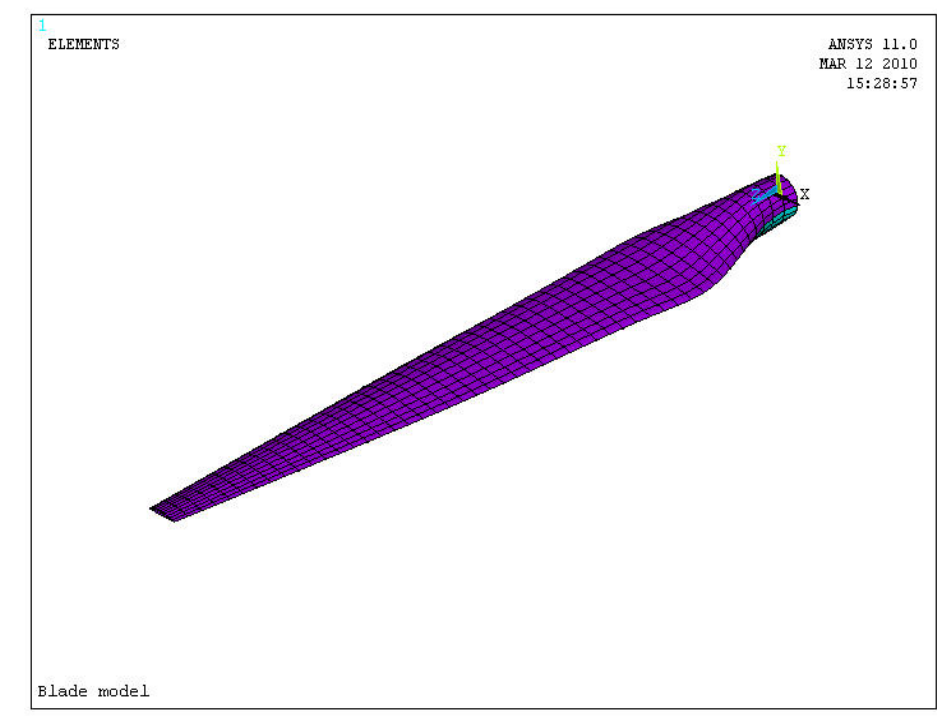

Figure AU-47: Blade model meshed with SHELL99 element. 
Intelligent Fiber Optic Sy stems Corp. (IFOS) DOE STTR GRANT No.: DE-

The model was examined under three standard cases of static loads: flexural, lateral, and torsional loads. Samples of the stress distribution in the global z-direction, $\mathrm{S}_{\mathrm{z}}$, are shown in Figures AU-48 to AU-50 for each case of loading. Three cases of loading were considered:

1. Two flexural loads at the tip of the blade in the global y-direction as shown in Figure AU-48.

2. Two lateral loads at the tip of the blade in the global $\mathrm{x}$-direction as shown in Figure $\mathrm{AU}-49$.

3. Two loads at the tip of the blade in opposing direction creating a torsional couple around the global z-direction as shown in Figure AU-50.

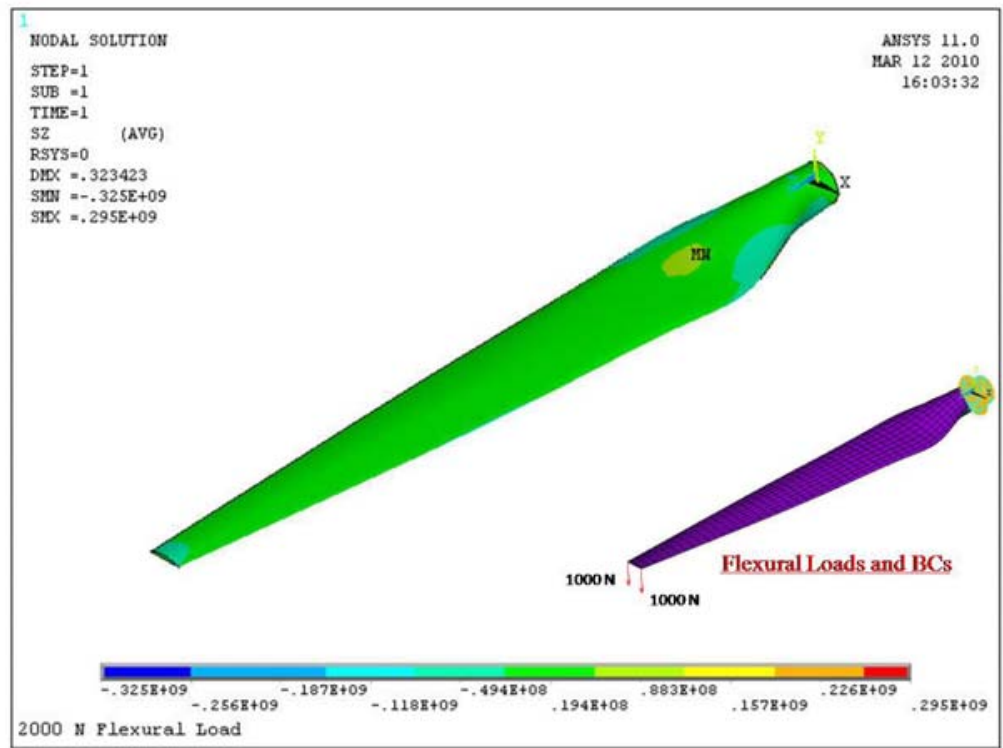

Figure AU-48: Stress distribution $S_{z}$ for the case of $2000 \mathrm{~N}$ flexural loads.

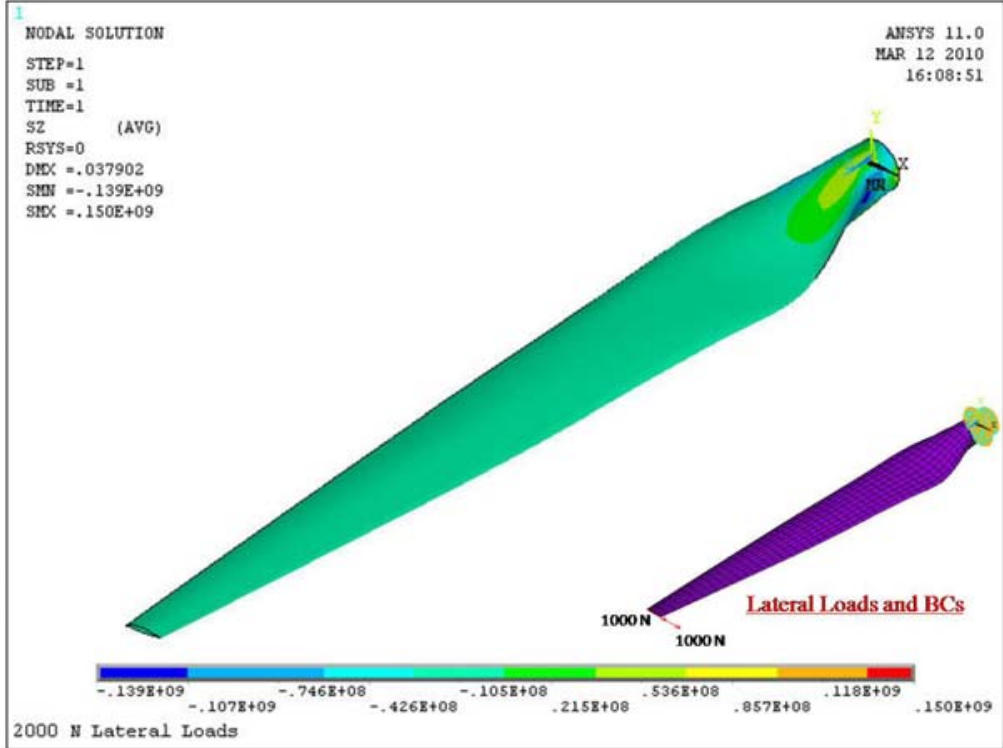


Intelligent Fiber Optic Sy stems Corp. (IFOS) DOE STTR GRANT No.: DE-

Figure AU-49: Stress distribution $S_{z}$ for the case of $2000 \mathrm{~N}$ lateral loads.

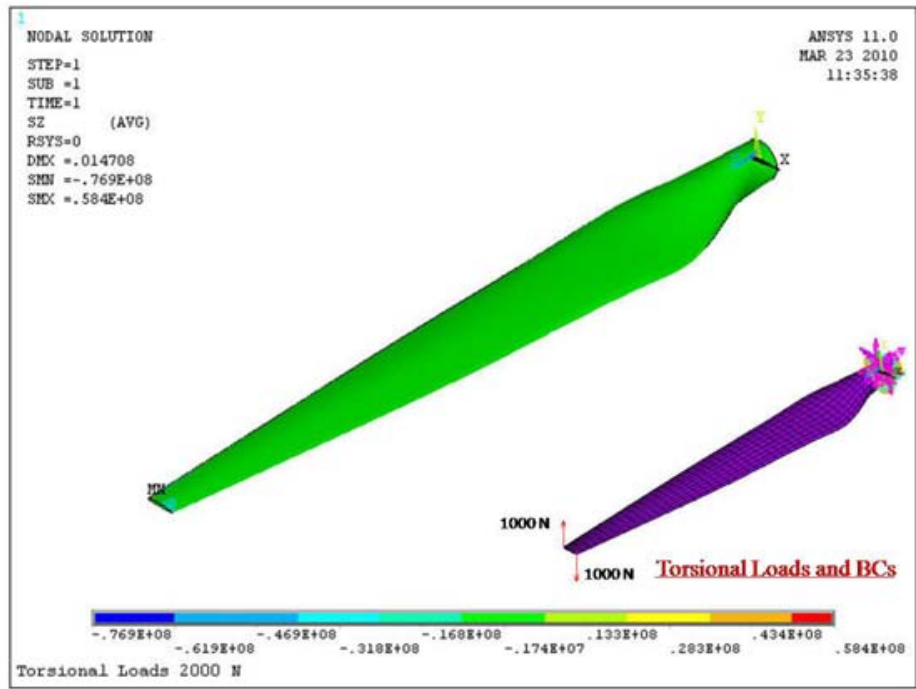

Figure AU-50: Stress distribution $\mathrm{S}_{\mathrm{z}}$ for the case of $2000 \mathrm{~N}$ torsional loads.

In order to locate best zones on the blade surface suitable for mounting or embedding the FBG sensors, maps for the distribution of main strain components were plotted for each case of loading. These components are: axial strain in the longitudinal and lateral directions $\left(\varepsilon_{z}\right.$ and $\varepsilon_{\mathrm{x}}$ ), and interfacial shear strain $\varepsilon_{\mathrm{xz}}$. These plots are shown in Figures AU-51 to AU-59 where preferable zones were marked with a white oval and also zones of local high strain concentration were marked as possible zones for FBG sensors mounting. These zones were selected as the zones with the highest strain values but with a minimum change of strain with distance. These locations would allow evaluation of critical strain values with a low chance of errors in sensor placement. 
Intelligent Fiber Optic Sy stems Corp. (IFOS) DOE STTR GRANT No.: DE-

Interim Report \#4

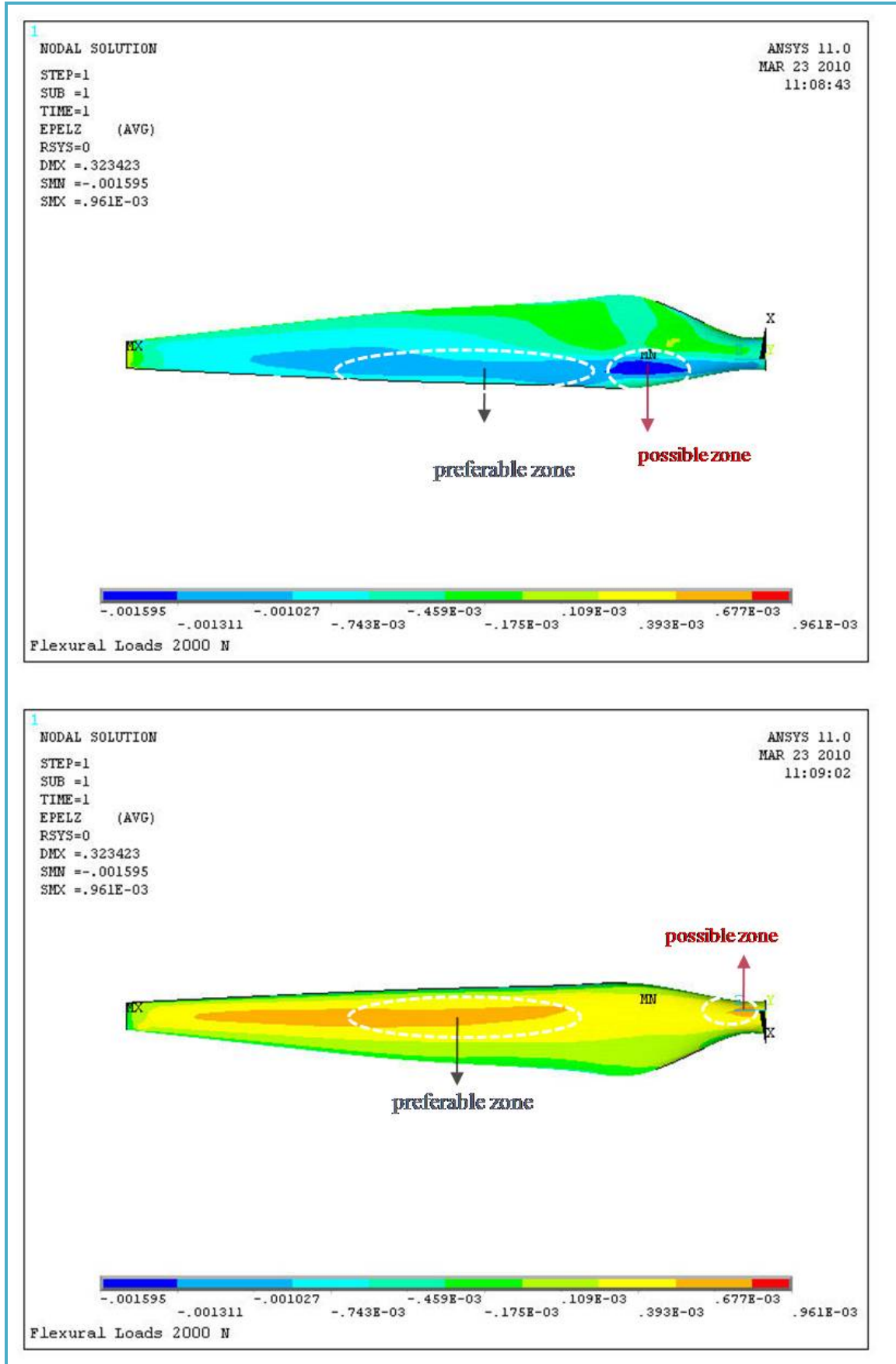

Figure AU-51: Recommended locations for mounting FBG sensors on upper and lower surfaces of blade based on the distribution of axial strain component $\varepsilon_{\mathrm{z}}$ for the case of $2000 \mathrm{~N}$ flexural loads. 
Intelligent Fiber Optic Sy stems Corp. (IFOS) DOE STTR GRANT No.: DE-

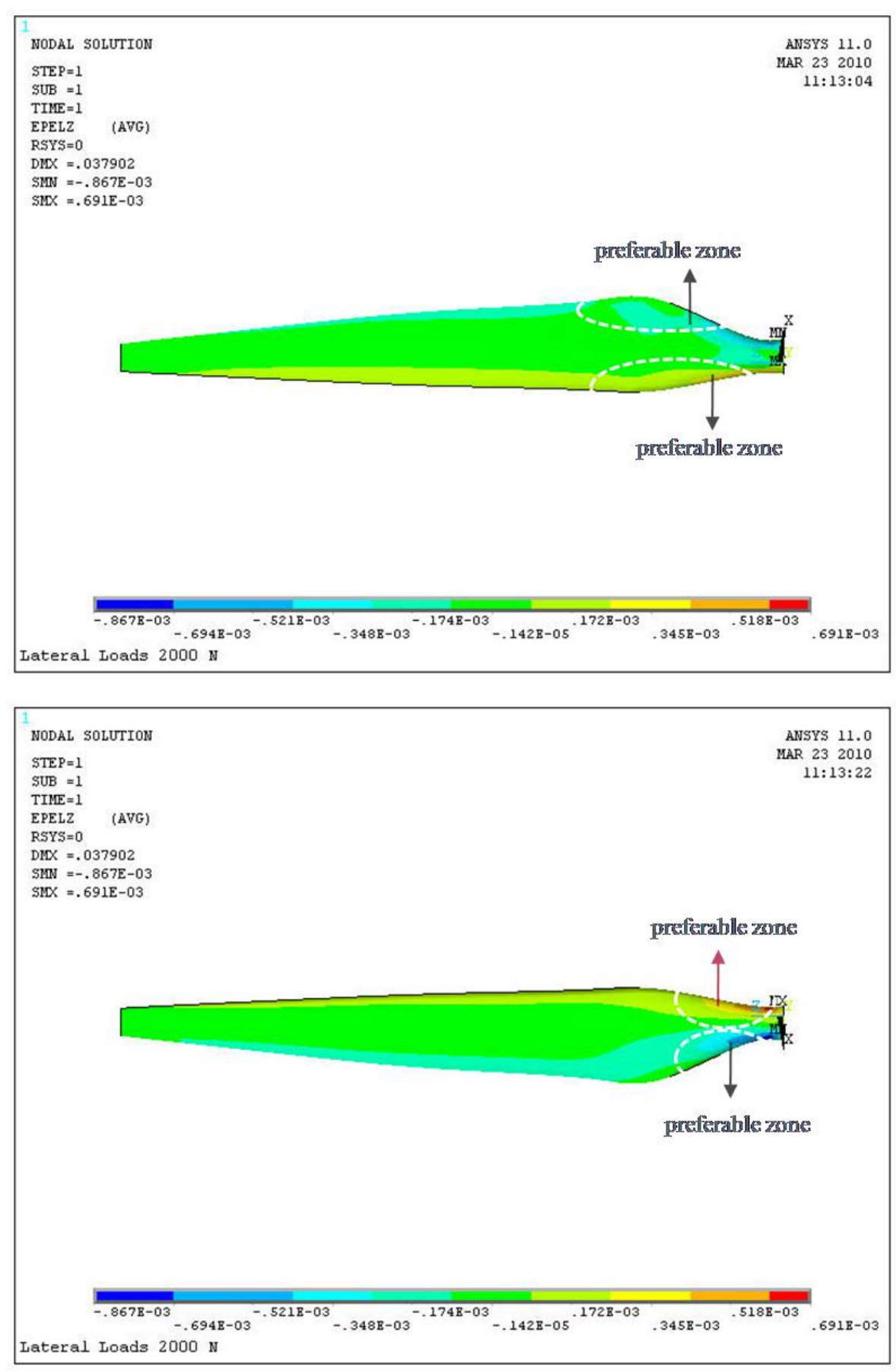

Figure AU-52: Recommended locations for mounting FBG sensors on upper and lower surfaces of blade based on the distribution of axial strain component $\varepsilon_{\mathrm{z}}$ for the case of $2000 \mathrm{~N}$ lateral loads. 
Intelligent Fiber Optic Sy stems Corp. (IFOS) DOE STTR GRANT No.: DE-

Interim Report \#4

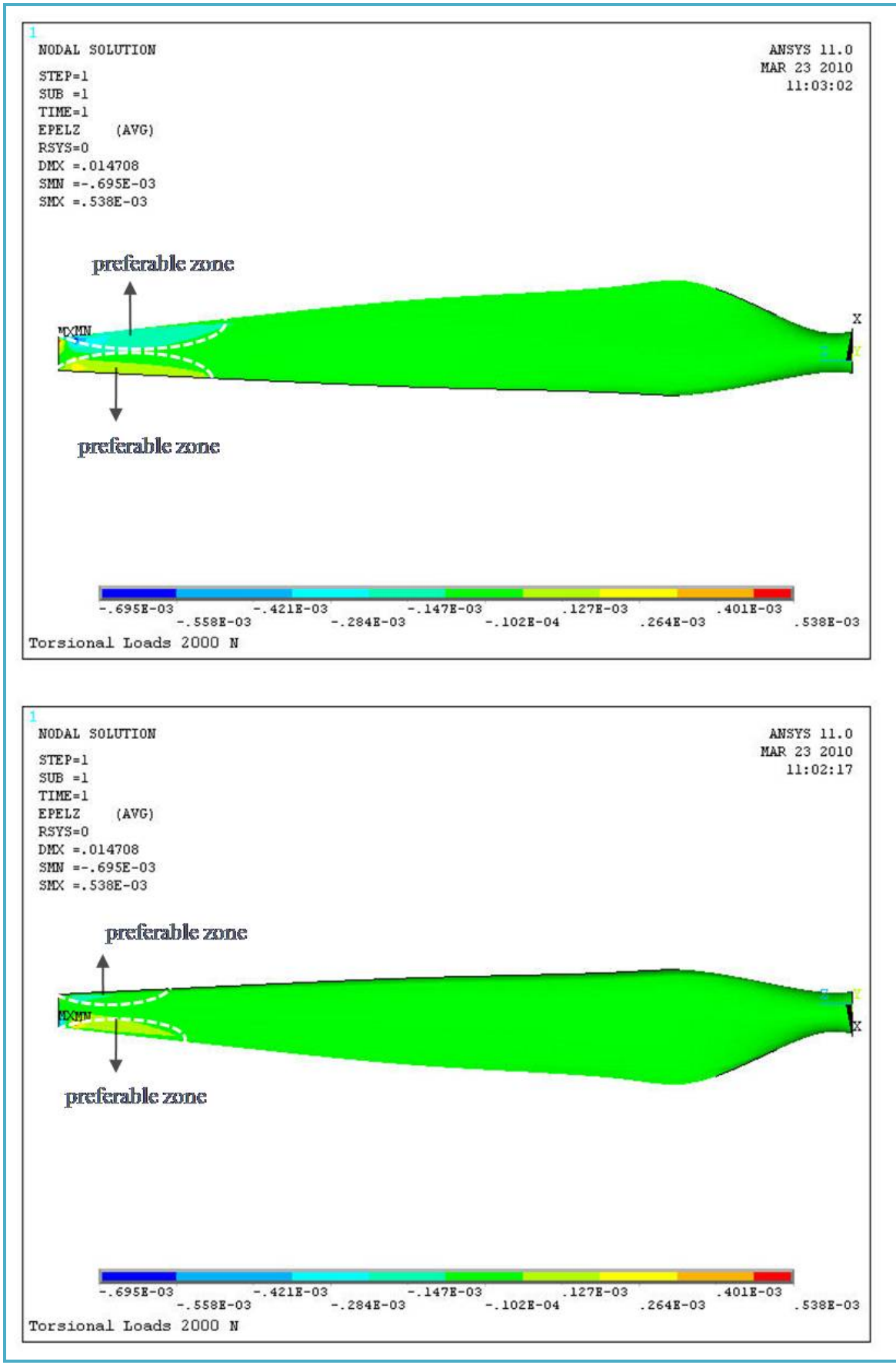

Figure AU-53: Recommended locations for mounting FBG sensors on upper and lower surfaces of blade based on the distribution of axial strain component $\varepsilon_{\mathrm{z}}$ for the case of $2000 \mathrm{~N}$ torsional loads. 
Intelligent Fiber Optic Sy stems Corp. (IFOS) DOE STTR GRANT No.: DE-

Interim Report \#4

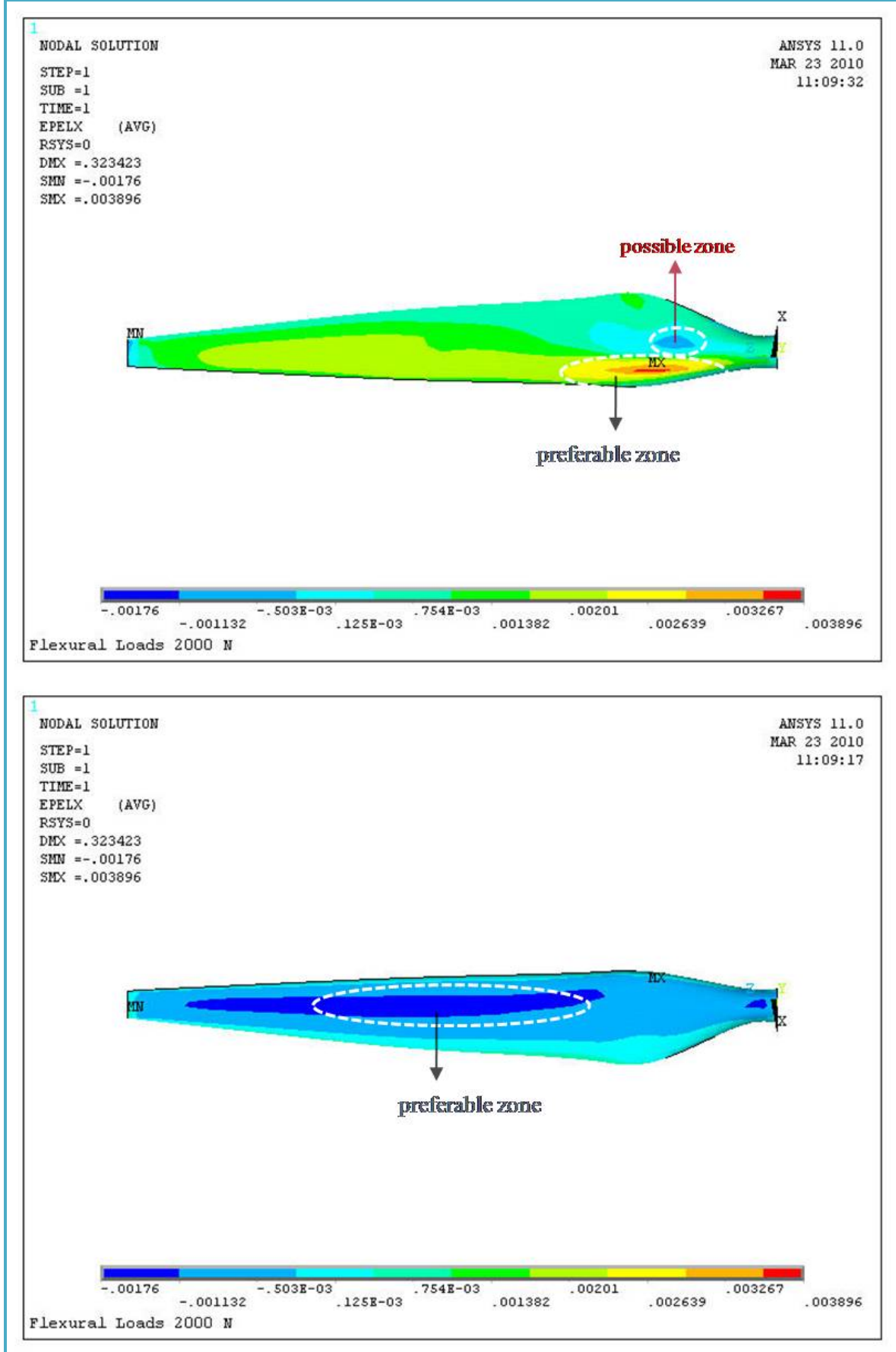

Figure AU-54: Recommended locations for mounting FBG sensors on upper and lower surfaces of blade based on the distribution of axial strain component $\varepsilon_{\mathrm{x}}$ for the case of $2000 \mathrm{~N}$ flexural loads. 
Intelligent Fiber Optic Sy stems Corp. (IFOS) DOE STTR GRANT No.: DE-

Interim Report \#4

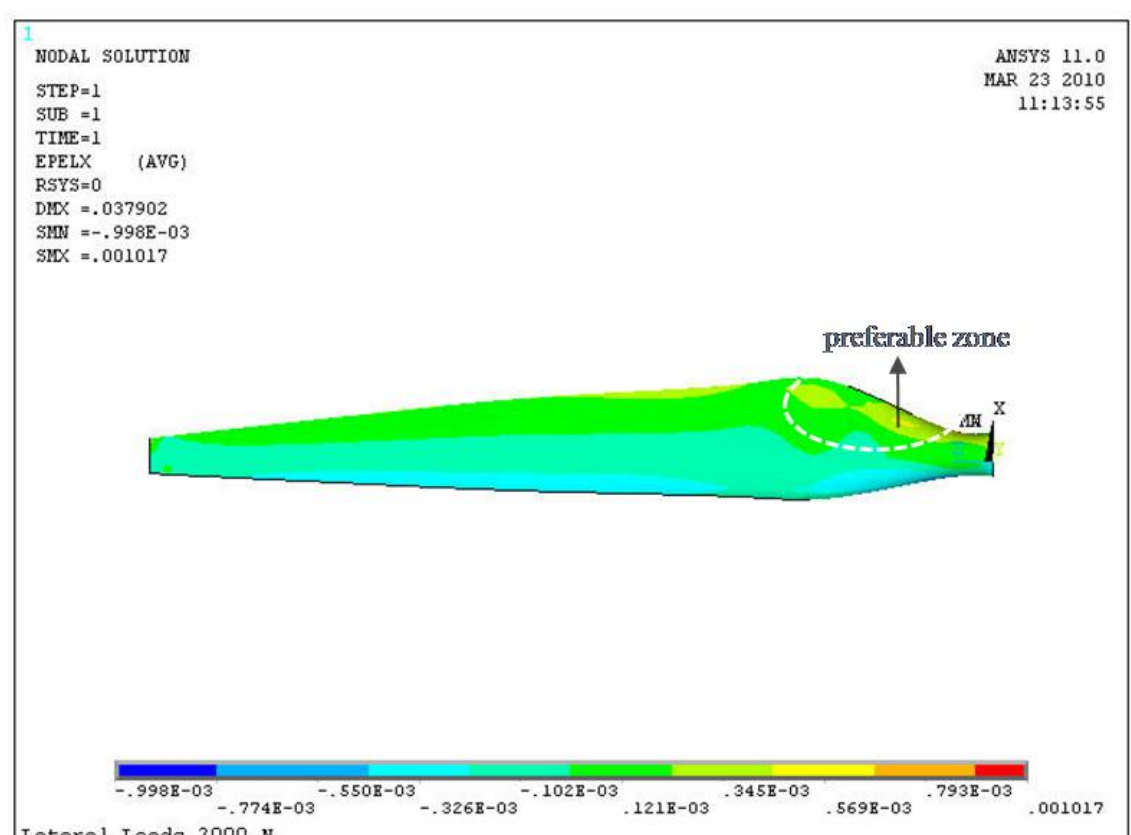

Lateral Loads $2000 \mathrm{~N}$

NODAL SOLUTION

$\operatorname{STEP}=1$

TI: $=1$

EPELX (AVG)

RSYS $=0$

DNX $=.037902$

SWN $=-.998 \mathrm{E}-03$

$\operatorname{SHX}=.001017$

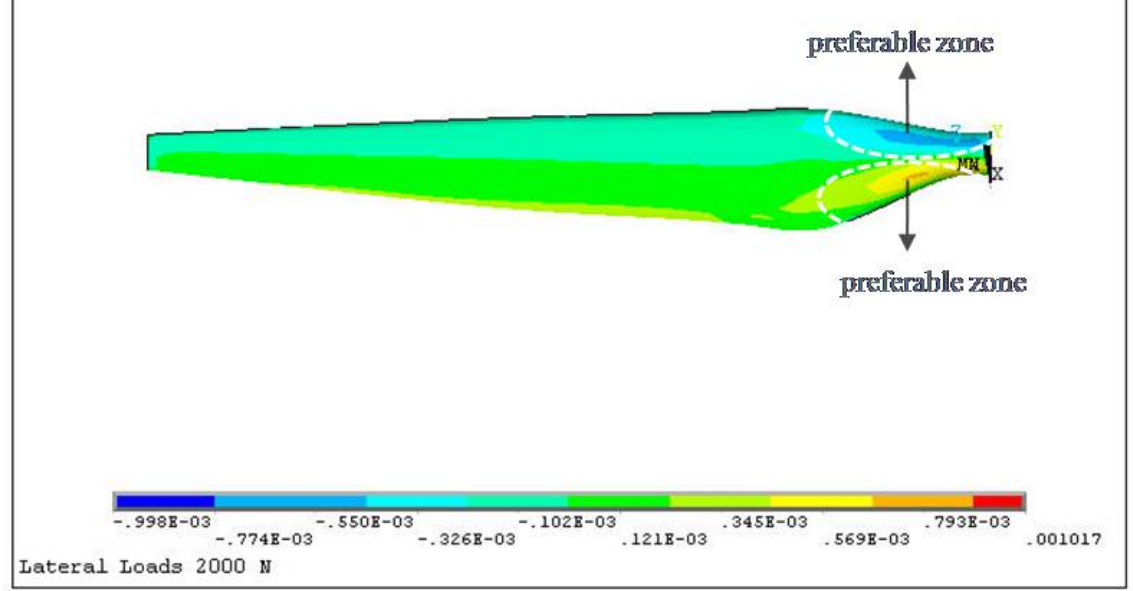

Figure AU-55: Recommended locations for mounting FBG sensors on upper and lower surfaces of blade based on the distribution of axial strain component $\varepsilon_{\mathrm{x}}$ for the case of $2000 \mathrm{~N}$ lateral loads. 
Intelligent Fiber Optic Sy stems Corp. (IFOS) DOE STTR GRANT No.: DE-

Interim Report \#4

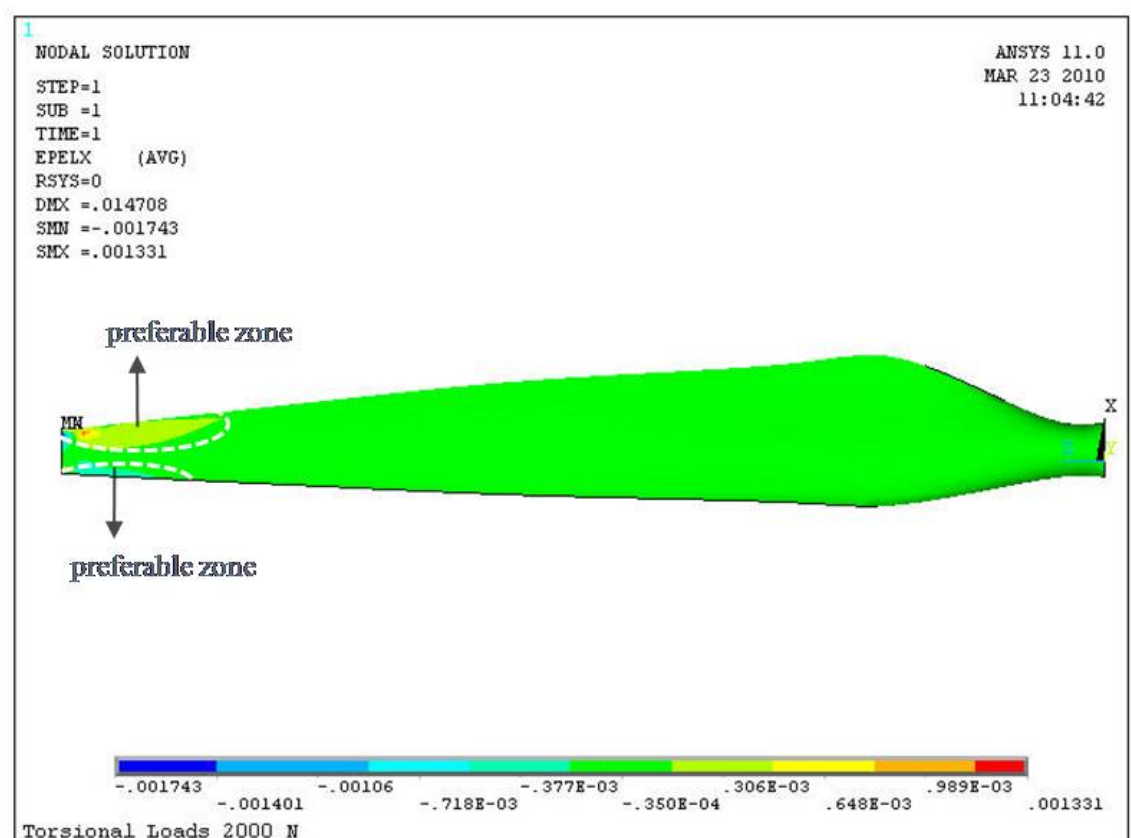

NODAL SOLUTION

STEP $=1$

SUB $=1$

TIME $=1$

EPELX (AVG)

RSYS $=0$

DHX $=.014708$

SMN $=-.001743$

$\operatorname{SLX}=.00133$

preferable zono

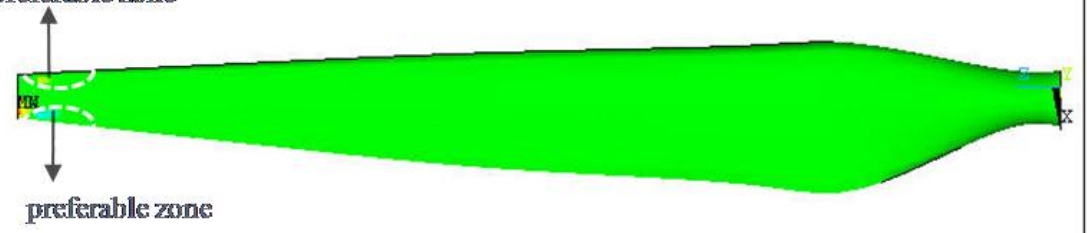

preferable zone

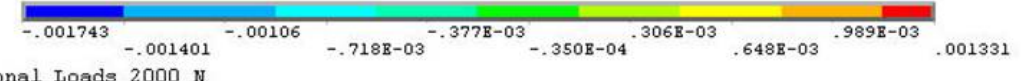

Torsional Loads $2000 \mathrm{~N}$

Figure AU-56: Recommended locations for mounting FBG sensors on upper and lower surfaces of blade based on the distribution of axial strain component $\varepsilon_{\mathrm{x}}$ for the case of $2000 \mathrm{~N}$ torsional loads. 
Intelligent Fiber Optic Sy stems Corp. (IFOS) DOE STTR GRANT No.: DE-

Interim Report \#4

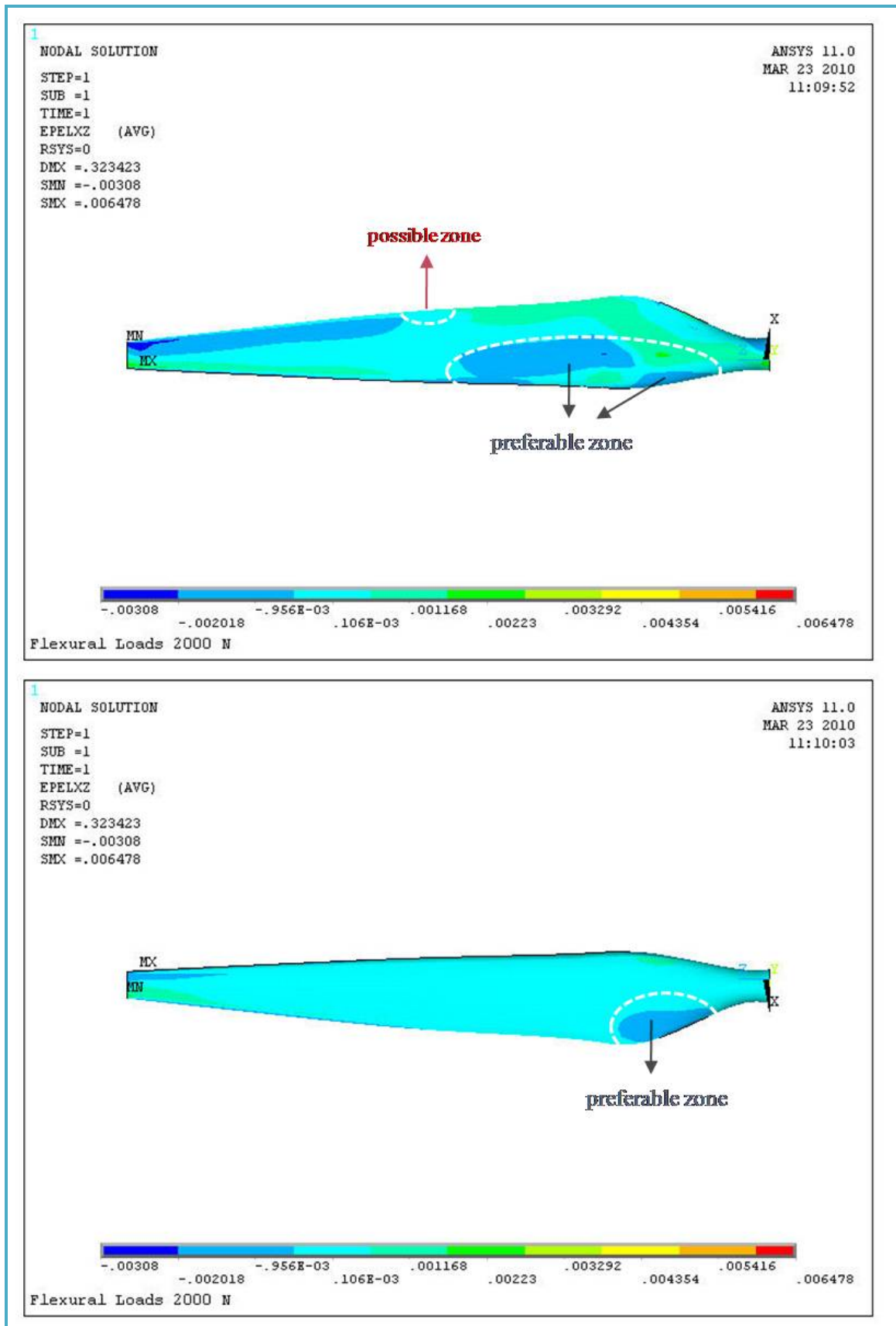

Figure AU-57: Recommended locations for mounting FBG sensors on upper and lower surfaces of blade based on the distribution of shear strain component $\varepsilon_{\mathrm{xz}}$ for the case of $2000 \mathrm{~N}$ flexural loads. 
Intelligent Fiber Optic Sy stems Corp. (IFOS) DOE STTR GRANT No.: DE-

Interim Report \#4

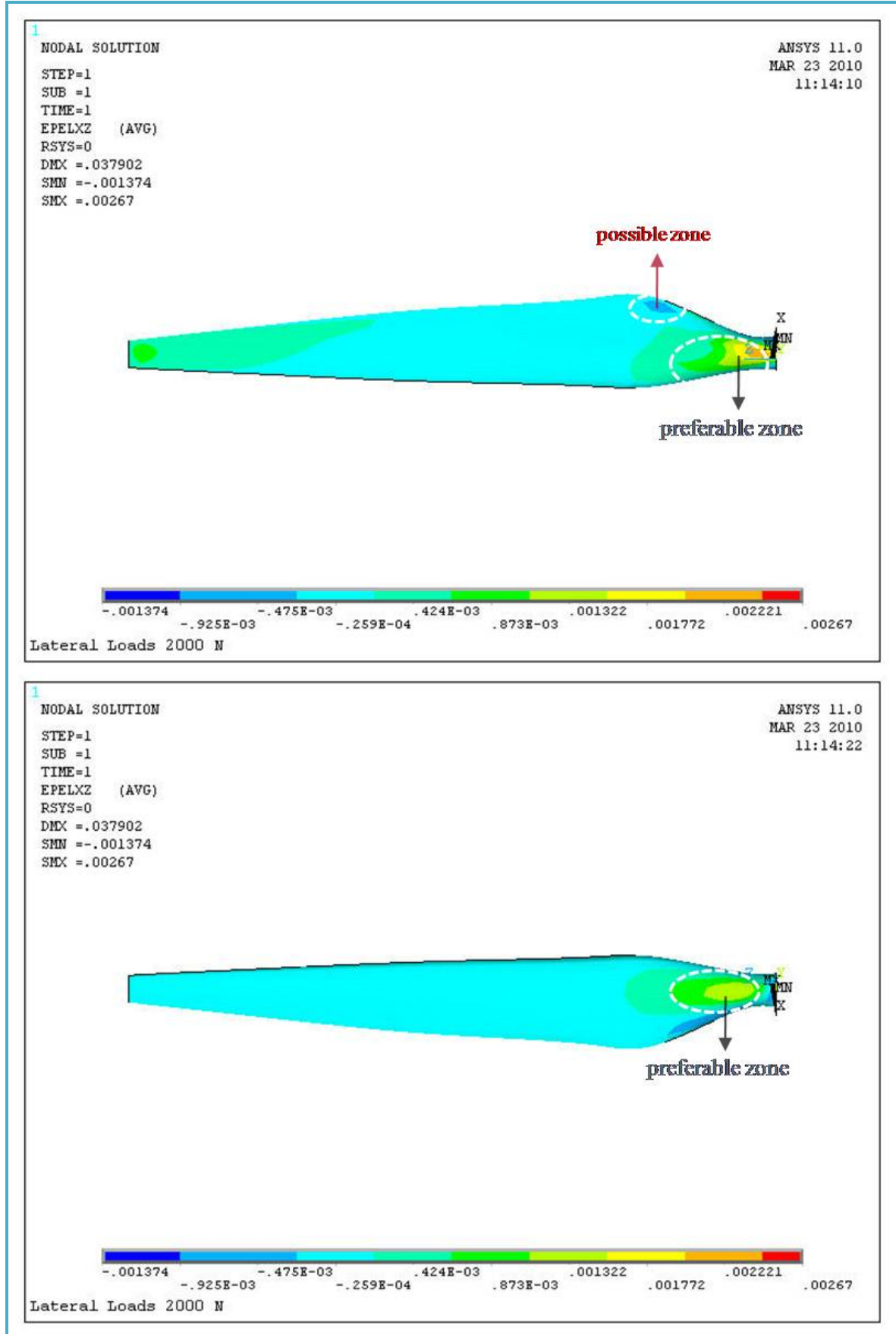

Figure AU-58: Recommended locations for mounting FBG sensors on upper and lower surfaces of blade based on the distribution of shear strain component $\varepsilon_{\mathrm{xz}}$ for the case of $2000 \mathrm{~N}$ lateral loads. 


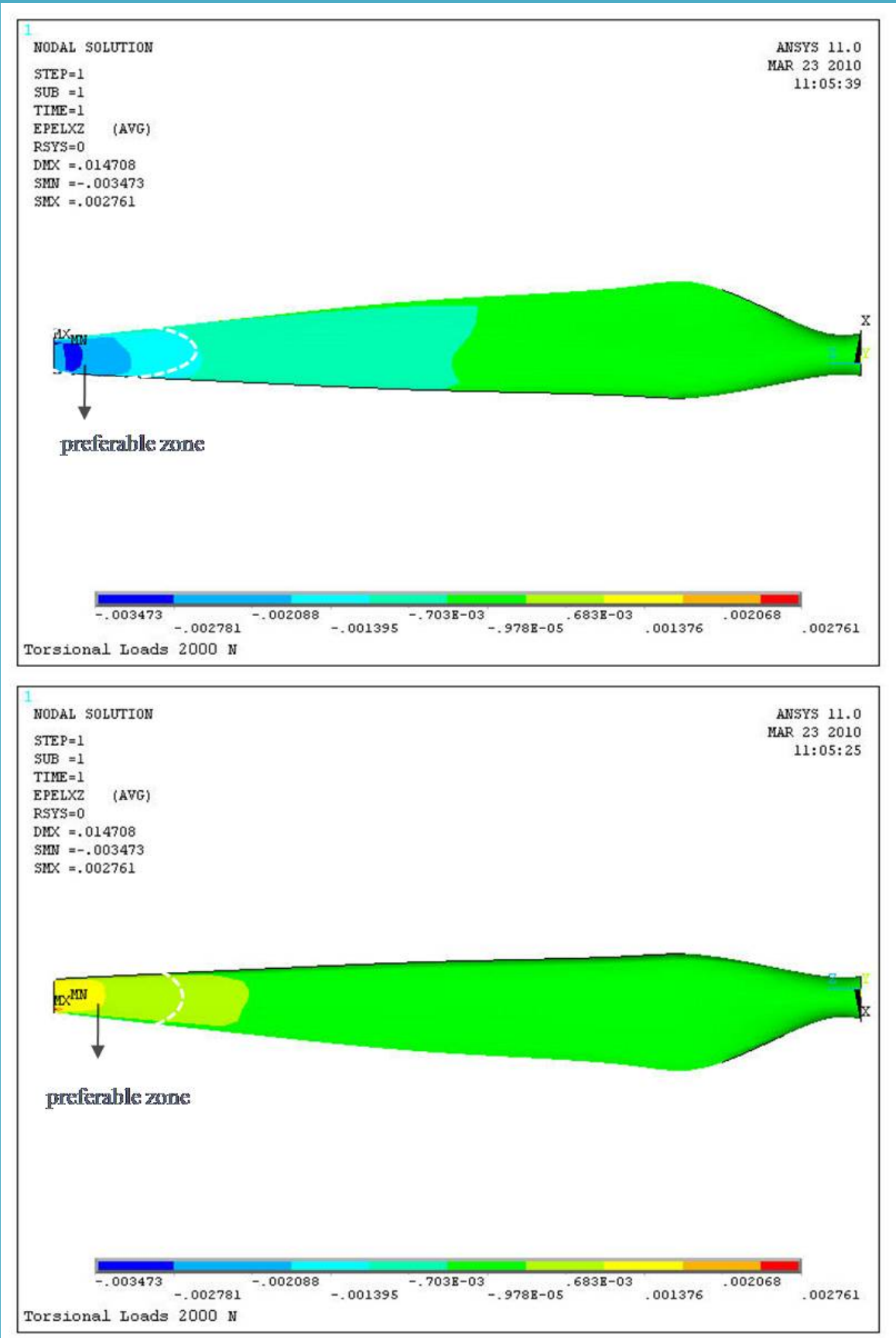

Figure AU-59: Recommended locations for mounting FBG sensors on upper and lower surfaces of blade based on the distribution of shear strain component $\varepsilon_{\mathrm{xz}}$ for the case of $2000 \mathrm{~N}$ torsional loads.

Based on these maps of strain distributions, shown in Figures AU-51 to AU-59, for this blade model it can be seen that preferable locations to mount the FBG sensors on the blade surface would be:

o Near the free end of the blade for the case of torsional loading.

0 At the transition part from the circular cross-section to the airfoil for the cases of lateral and flexural loadings. 
Intelligent Fiber Optic Sy stems Corp. (IFOS) DOE STTR GRANT No.: DE-

o In the middle of the blade for the case of flexural loading. However, it should be noted that these locations may vary for other blade models depending on their geometries and applied loading conditions. Also, some of these load condition will happen concurrently forcing a combined scheme for sensor placement.

\section{Conclusions}

1. The experimental program, performed by AU in this project, proved the high accuracy of the FBG sensors in detecting elastic response of the tested composite coupons. Under different static and dynamic cases of loading, strain measurements of FBG sensors were in close agreement to those of standard experimental devices, such as the video extensometer and laser vibrometer.

2. Results of FEA models of tested coupons matched experimental results providing confidence in the material properties incorporated in the model.

3. It was observed that the introduction of the defect in the composite coupon caused a change of natural frequency from $52.3 \mathrm{~Hz}$ to $56 \mathrm{~Hz}$.

4. A turbine wind blade was modeled using FEA. The model was used to propose possible locations for FBG sensors based on maps of strain distribution under three standard cases of loading.

\section{Recommendations for Phase II Research}

1. Invite a turbine blade manufacturer to join the research team and implement this experimental program on an actual composite material turbine wind blade.

2. Run FEA models of coupons and evaluate damping parameters.

3. Create FEA models of actual blades and decide on locations of FBG sensors.

4. Investigate effect of embedding sensors vs. attaching them to the surface of the blades.

5. Test dynamic properties of blades produced by manufacturer with embedded and mounted sensors.

6. Down-select to either embedding or mounting of sensors.

7. Run static and dynamic tests on an instrumented blade and compare results to FEA models. 


\section{REPORT DOCUMENTATION PAGE}

Form Approved

OMB No. 0704-0188

Public reporting burden for this collection of information is estimated to average 1 hour per response, including the time for reviewing instructions, searching existing data sources, gathering and maintaining the data needed, and completing and reviewing the collection of information. Send comments regarding this burden estimate or any other aspect of this collection of information, including suggestions for reducing this burden, to Department of Defense, Washington Headquarters Services, Directorate for Information Operations and Reports (0704-0188), 1215 Jefferson Davis Highway, Suite 1204, Arlington, VA 22202-4302. Respondents should be aware that notwithstanding any other provision of law, no person shall be subject to any penalty for failing to comply with a collection of information if it does not display a currently valid OMB control number.

PLEASE DO NOT RETURN YOUR FORM TO THE ABOVE ADDRESS.

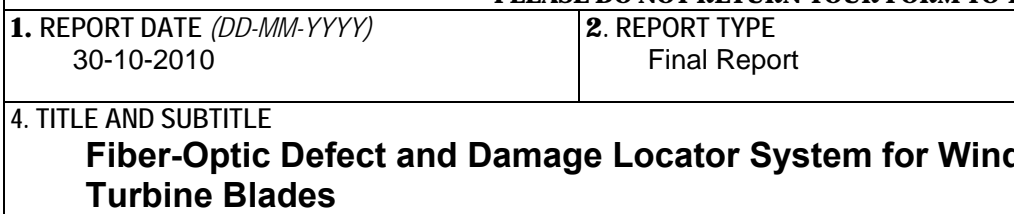

3. DATES COVERED (From - To)

19-04-2010 to 20-07-2009

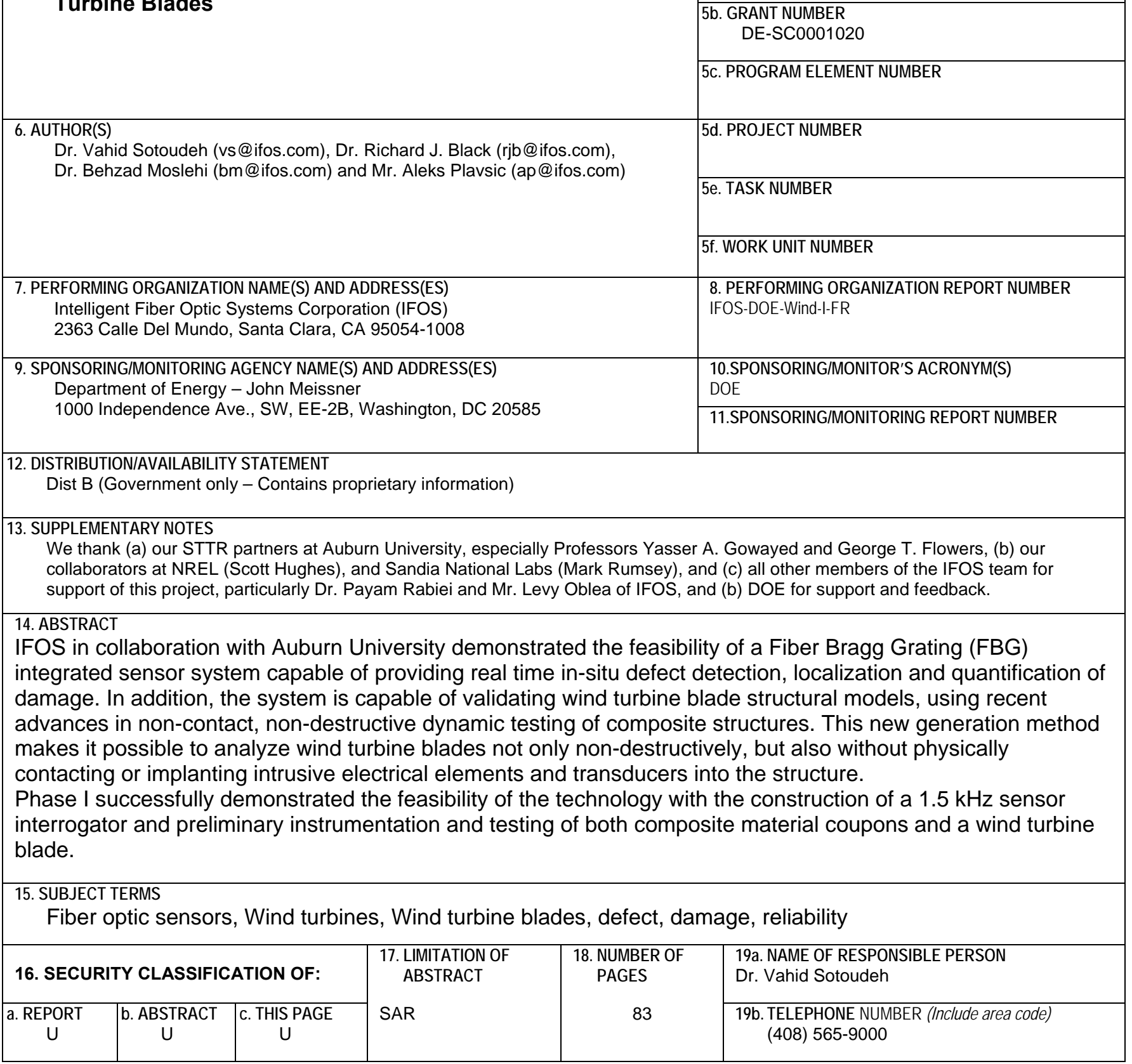

